\title{
Supporting Information for Catalytic Diastereoselective Polycyclization of Homo(polyprenyl)arene Analogues Bearing Terminal Siloxyvinyl Groups
}

Muhammet Uyanik, ${ }^{\dagger}$ Kazuaki Ishihara, ${ }^{* \dagger}$ and Hisashi Yamamoto*,*

Graduate School of Engineering, Nagoya University, Furo-cho, Chikusa, Nagoya 464-8603, Japan, and Department of Chemistry, The University of Chicago, 5735 South Ellis Avenue, Chicago, Illinois 60637

E-mail: ishihara@cc.nagoya-u.ac.jp; yamamoto@uchicago.edu

\section{Table of Contents:}

General Methods S1

Synthesis of Starting Materials S1

General Procedure for the $\mathrm{SnCl}_{4}$-Promoted Cyclization S23

Determination of the Relative Stereochemistry of Polycyclic Products S35

References and Notes S40

Spectroscopic Data

General Methods. Infrared (IR) spectra were recorded on a JASCO FT/IR 460 plus spectrometer. ${ }^{1} \mathrm{H}$ NMR spectra were measured on a Varian Gemini-2000 (300 MHz) or Varian INOVA-500 (500 $\mathrm{MHz}$ ) spectrometer at ambient temperature. Data were recorded as follows: chemical shift in ppm from internal tetramethylsilane on the $\delta$ scale, multiplicity $(\mathrm{s}=\operatorname{singlet} ; \mathrm{d}=\mathrm{doublet} ; \mathrm{t}=$ triplet; $\mathrm{q}=$ quartet; $\mathrm{m}$ $=$ multiplet $)$, coupling constant $(\mathrm{Hz})$, integration, and assignment. ${ }^{13} \mathrm{C}$ NMR spectra were measured on a Varian Gemini-2000 (75 MHz) or Varian INOVA-500 (125 MHz) spectrometer. Chemical shifts were recorded in ppm from the solvent resonance employed as the internal standard (deuterochloroform at $77.00 \mathrm{ppm}$ ). High-performance liquid chromatography (HPLC) analysis was conducted using Shimadzu LC-10 AD coupled diode array-detector SPD-MA-10A-VP and chiral column of Daicel CHIRALCEL OD-H (4.6 mm x $25 \mathrm{~cm})$. Low-resolution mass analysis (LRMS) and GC analysis were performed with a Shimadzu GC/MS instrument [GC-17A/QP-5050A; column: TC-1 (0.25 mm x $30 \mathrm{~m})$ ] by direct insertion for chemical ionization (CI) with isobutane. The temperature program employed was $70{ }^{\circ} \mathrm{C}$ for $5 \mathrm{~min}$ with an increase to $250{ }^{\circ} \mathrm{C}$ at $10^{\circ} \mathrm{C} / \mathrm{min}$. For thin-layer chromatography (TLC) analysis throughout this work, Merck precoated TLC plates (silica gel $60 \mathrm{GF}_{254} 0.25 \mathrm{~mm}$ ) were used. The products were purified by column chromatography on silica gel (E. Merck Art. 9385) or aluminum oxide 150 basic (type T) (Merck). High-resolution mass spectral analysis (HRMS) was performed at Chemical Instrument Center, Nagoya University. In experiments that required dry solvent, ether and tetrahydorofuran (THF) were purchased from Aldrich or Wako as the "anhydrous" and stored over 4A molecular sieves. Hexane, toluene and dichloromethane were freshly distilled from calcium hydride. Tin(IV) chloride was distilled under nitrogen. Other simple chemicals were analytical-grade and obtained commercially.

\section{Synthesis of Starting Materials.}

Scheme 3. Preparation of Silyl Polyenol Ethers ${ }^{a}$ 
<smiles>CC(=O)CC1=C(C)CCC=C1C</smiles>

25a $(n=1,2 E)$

25b $(n=1,2 Z)$

25c $(n=2,2 E)$<smiles>CC(=O)OCC=C(C)CCC1OC1(C)C</smiles>

26a $(n=1,2 E)$ 26b $(n=1,2 Z)$ 26c $(n=2,2 E)$

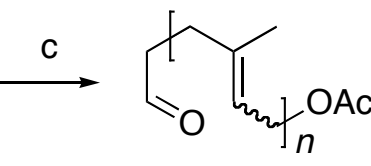

27a $(n=1,2 E)$

27b $(n=1,2 Z)$ : $96 \%$ from $25 \mathbf{b}$ 27c $(n=2,2 E)$

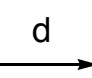<smiles>CC(=O)OCCO</smiles>

28a $(n=1,2 E$

28b $(n=1,2 Z): 96 \%$ from 27c

28c $(n=2,2 E): 96 \%$ from 25c

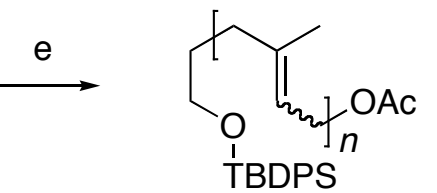

29a $(n=1,2 E)$ 29b $(n=1,2 Z): 98 \%$ from $\mathbf{2 8 b}$ 29c $(n=2,2 E)$

$\mathrm{h}$<smiles>CC(=CCCCCCCO)Cc1ccccc1</smiles>

32aa $(n=1,4 E, \mathrm{R}=p-\mathrm{F})$ : >99\% from 31a 32ab ( $n=1,4 E, \mathrm{R}=\mathrm{H})$ : 97\% from 31a 32ac $(n=1,4 E, \mathrm{R}=p-\mathrm{Me})$ : $95 \%$ from $31 \mathrm{a}$ 32ad ( $n=1,4 E, \mathrm{R}=m-\mathrm{Me})$ : $94 \%$ from 31a 32ae $(n=1,4 E, \mathrm{R}=m-\mathrm{IPr})$ : $95 \%$ from 31a 32ba $(n=1,4 Z, \mathrm{R}=p-\mathrm{Me})$ : $94 \%$ from 31b 32bb $(n=1,4 Z, \mathrm{R}=m-\mathrm{Me})$ : $94 \%$ from 31b 32ca $(n=2,8 E, \mathrm{R}=p-\mathrm{Me})$ : $99 \%$ from 31c 32cb ( $n=2,8 E, \mathrm{R}=m-\mathrm{Me}): 97 \%$ from 31c

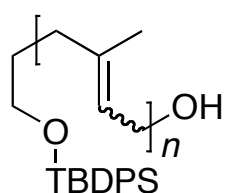

30a $(n=1,2 E): 80 \%$ from $25 \mathbf{a}$ 30b $(n=1,2 Z): 98 \%$ from $29 \mathbf{b}$ 30c $(n=2,2 E)$ : $95 \%$ from $\mathbf{2 8 c}$<smiles>CCOCCOCCOCCOCC</smiles>
TBDPS

31a $(n=1,2 E)$ : $90 \%$ from 30a 31b $(n=1,2 Z): 98 \%$ from 30b 31c $(n=2,2 E): 91 \%$ from 30 c

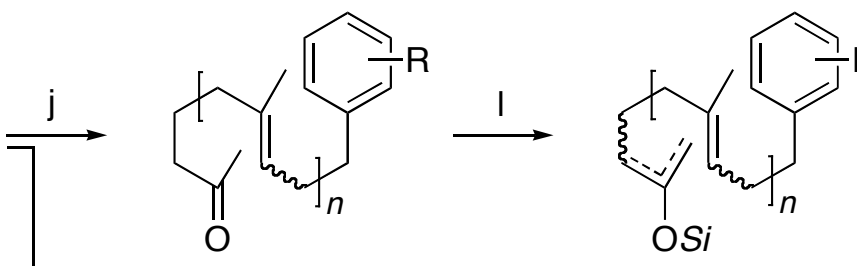

33aa $(n=1,5 E, \mathrm{R}=p-\mathrm{F})$

33ab $(n=1,5 E, \mathrm{R}=\mathrm{H})$

33ac $(n=1,5 E, \mathrm{R}=p-\mathrm{Me})$ : $95 \%$ from 32ac

33ad $(n=1,5 E, \mathrm{R}=m-\mathrm{Me}): 96 \%$ from 32ad

33ae $(n=1,4 E, \mathrm{R}=m$-IPr): $95 \%$ from 32ae

33ba $(n=1,5 Z, \mathrm{R}=p-\mathrm{Me}): 98 \%$ from $32 \mathrm{ba}$

33bb ( $n=1,5 Z, \mathrm{R}=m-\mathrm{Me}): 98 \%$ from $3 \mathbf{b b}$

33ca $(n=2,9 E, \mathrm{R}=p-\mathrm{Me})$ : $97 \%$ from 32ca

33cb ( $n=2,9 E, \mathrm{R}=m-\mathrm{Me}): 93 \%$ from 32cb

34aa $(n=1,6 E, \mathrm{R}=p-\mathrm{F})$ : $96 \%$ from 32aa 34ab $(n=1,6 E, \mathrm{R}=\mathrm{H}): 96 \%$ from 32ab 34ac $(n=1,6 E, \mathrm{R}=p-\mathrm{Me})$ : $94 \%$ from 33ac $1(n=1,6 E, \mathrm{R}=m-\mathrm{Me}): 97 \%$ from 33ad 34ae $(n=1,4 E, \mathrm{R}=m-i-\operatorname{Pr}): 96 \%$ from 33ae 34ba $(n=1,6 Z, \mathrm{R}=p-\mathrm{Me}): 94 \%$ from $33 \mathrm{ba}$ 34bb ( $n=1,6 Z, \mathrm{R}=m-\mathrm{Me})$ : $95 \%$ from 33bb 34ca $(n=2,10 E, \mathrm{R}=p-\mathrm{Me}): 95 \%$ from 33ca 34cb $(n=2,10 E, \mathrm{R}=m-\mathrm{Me})$ : $96 \%$ from 33cb 4a $(n=1,6 E, \mathrm{R}=p-\mathrm{F}, \mathrm{Si}=\mathrm{TIPS}):>99 \%$ from $34 \mathrm{aa}$ 4b $(n=1,6 E, \mathrm{R}=\mathrm{H}, \mathrm{Si}=\mathrm{TIPS}):>99 \%$ from 34ab 4c $(n=1,6 E, \mathrm{R}=p-\mathrm{Me}, \mathrm{Si}=\mathrm{TIPS})$ : $>99 \%$ from 34ac 4d $(n=1,6 E, \mathrm{R}=m-\mathrm{Me}, \mathrm{Si}=\mathrm{TIPS}):>99 \%$ from 1 4e $(n=1,6 E, \mathrm{R}=p-\mathrm{Me}, \mathrm{Si}=\mathrm{TBDPS}): 84 \%$ from $34 \mathrm{ac}$ $4 f(n=1,6 E, \mathrm{R}=m-\mathrm{Me}, \mathrm{Si}=\mathrm{TBDPS}): 65 \%$ from 1 4h ( $n=1,4 E, \mathrm{R}=m-i-\operatorname{Pr}, S i=T B D P S) 93 \%$ from $34 a e$ 7a $(n=1,6 Z, \mathrm{R}=p-\mathrm{Me}, \mathrm{Si}=\mathrm{TIPS}): 98 \%$ from $34 \mathrm{ba}$ 7b $(n=1,6 Z, \mathrm{R}=m-\mathrm{Me}, \mathrm{Si}=\mathrm{TIPS}):>99 \%$ from $34 \mathrm{bb}$ 7c ( $n=1,6 Z, \mathrm{R}=m-\mathrm{Me}, \mathrm{Si}=\mathrm{TBDPS}): 92 \%$ from $\mathbf{3 4 b b}$ 10a $(n=2,10 E, \mathrm{R}=p-\mathrm{Me}, \mathrm{Si}=\mathrm{TIPS})$ : $>99 \%$ from 34ca 10b $(n=2,10 E, \mathrm{R}=m-\mathrm{Me}, \mathrm{Si}=\mathrm{TIPS}):>99 \%$ from $\mathbf{3 4 \mathbf { c b }}$ 10c $(n=2,10 E, \mathrm{R}=m-\mathrm{Me}, \mathrm{Si}=\mathrm{TBDPS}): 81 \%$ from $34 \mathbf{c b}$ $\mathrm{k}$

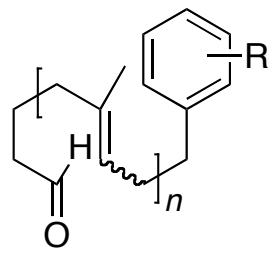

13a $(\mathrm{R}=p-\mathrm{Me}): 94 \%$ from 33ac 13b ( $\mathrm{R}=m-\mathrm{Me}): 96 \%$ from 33ad 13c $(\mathrm{R}=m-i-\mathrm{Pr}): 93 \%$ from 33ae

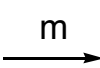<smiles>CCCC=C(C)CCCC(C)=CCCc1ccccc1</smiles>

$14 a(R=p-M e):>95 \%$ yield from $13 a$ 14b $(\mathrm{R}=m-\mathrm{Me}):>95 \%$ yield from $13 \mathrm{~b}$ 14c $(\mathrm{R}=m-i-\mathrm{Pr}):>95 \%$ yield from 13c 
${ }^{a}$ Reagents and conditions: (a) $m \mathrm{CPBA}, \mathrm{CH}_{2} \mathrm{Cl}_{2},-20{ }^{\circ} \mathrm{C}$ to rt, $2 \mathrm{~h}$; (b) $i$. NBS, THF- $\mathrm{H}_{2} \mathrm{O},-5^{\circ} \mathrm{C}$ to $0{ }^{\circ} \mathrm{C}$, 3 h; ii. $\mathrm{K}_{2} \mathrm{CO}_{3}, \mathrm{MeOH}$, rt, $2 \mathrm{~h}$; iii. $\mathrm{Ac}_{2} \mathrm{O}$, pyridine, rt, $1 \mathrm{~d}$; (c) $\mathrm{HIO}_{4} \bullet 2 \mathrm{H}_{2} \mathrm{O}$, THF-Et $2 \mathrm{O}, 0{ }^{\circ} \mathrm{C}, 30$ min.; (d) $\mathrm{NaBH}_{4}, \mathrm{EtOH}, 0{ }^{\circ} \mathrm{C}, 1 \mathrm{~h}$; (e) TBDPSCl, Imidazole, DMF, rt, on; (f) $\mathrm{K}_{2} \mathrm{CO}_{3}, \mathrm{MeOH}, 0{ }^{\circ} \mathrm{C}$ to rt, $1 \mathrm{~h}$; (g) (EtO) ${ }_{2} \mathrm{POCl}, \mathrm{Et}_{3} \mathrm{~N}$, hexane, $0{ }^{\circ} \mathrm{C}$ to rt, $1 \mathrm{~d}$; (h) $i$. ArMgX, THF, $0{ }^{\circ} \mathrm{C}$ to rt, on; $i i$. TBAF, THF, rt, on; (i) $i$. $\mathrm{MsCl}, \mathrm{Et}_{3} \mathrm{~N}, \mathrm{CH}_{2} \mathrm{Cl}_{2}, 0^{\circ} \mathrm{C}$ for $1 \mathrm{~h}$, rt for 30 min.; ii. $\mathrm{KCN}, 18$-crown-6, $\mathrm{CH}_{3} \mathrm{CN}$, reflux, 3-6 h; (j) MeLi, $\mathrm{Et}_{2} \mathrm{O},-78{ }^{\circ} \mathrm{C}$ to $0{ }^{\circ} \mathrm{C}$, $1 \mathrm{~h}$; (k) DIBAL-H, hexane-toluene, $-78{ }^{\circ} \mathrm{C}, 1 \mathrm{~h}$; (l) $\operatorname{SiOTf}, \mathrm{Et}_{3} \mathrm{~N}, \mathrm{CH}_{2} \mathrm{Cl}_{2}, 0{ }^{\circ} \mathrm{C}$ to rt, 3-12 h; (m) TIPSOTf, $\mathrm{Et}_{3} \mathrm{~N}-\mathrm{CH}_{2} \mathrm{Cl}_{2},-78^{\circ} \mathrm{C}$ to $0{ }^{\circ} \mathrm{C}, 2 \mathrm{~h}$.

General Procedure for the Regioselective Epoxidation of 25a and 25b (Step a, Scheme 3). To a stirring solution of $25(50.0 \mathrm{mmol})$ in $\mathrm{CH}_{2} \mathrm{Cl}_{2}(100 \mathrm{~mL})$ at $-20{ }^{\circ} \mathrm{C}$ was added a solution of $m \mathrm{CPBA}$

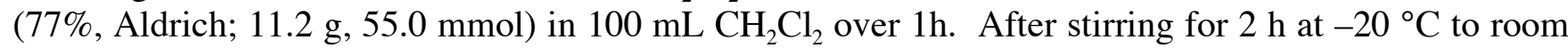
temperature, the reaction was quenched with $3 \mathrm{M} \mathrm{NaOH}(25 \mathrm{~mL})$. The aqueous layer was extracted with $\mathrm{CH}_{2} \mathrm{Cl}_{2}(3 \times 20 \mathrm{~mL})$ and the combined organic layers were washed with brine, dried over anhydrous $\mathrm{MgSO}_{4}$ and concentrated in vacuo to give $\mathbf{2 6}$ in quantitative yield. The crude product was used for next step without further purification.

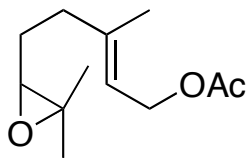

(E)-5-(3,3-Dimethyloxiran-2-yl)-3-methylpent-2-enyl Acetate (26a): ${ }^{1}$ TLC, $R_{\mathrm{f}}=0.42$ (hexane-EtOAc $=4: 1) ;{ }^{1} \mathrm{H}$ NMR $\left(\mathrm{CDCl}_{3}, 300 \mathrm{MHz}\right) \delta 1.27(\mathrm{~s}, 3 \mathrm{H}), 1.31(\mathrm{~s}, 3 \mathrm{H}), 1.60-1.73(\mathrm{~m}, 2 \mathrm{H})$, $1.73(\mathrm{~s}, 3 \mathrm{H}), 2.06(\mathrm{~s}, 3 \mathrm{H}), 2.06-2.26(\mathrm{~m}, 2 \mathrm{H}), 2.71(\mathrm{t}, J=6.3 \mathrm{~Hz}, 1 \mathrm{H}), 4.59(\mathrm{~d}, J=7.2 \mathrm{~Hz}, 2 \mathrm{H}), 5.39$ (t, $J=6.6 \mathrm{~Hz}, 1 \mathrm{H})$.

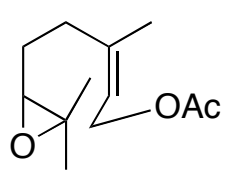

(Z)-5-(3,3-Dimethyloxiran-2-yl)-3-methylpent-2-enyl Acetate (26b): ${ }^{1 \mathrm{~b}, 2}$ TLC, $R_{\mathrm{f}}=0.43$ (hexane-EtOAc $=4: 1) ;{ }^{1} \mathrm{H}$ NMR $\left(\mathrm{CDCl}_{3}, 300 \mathrm{MHz}\right) \delta 1.27(\mathrm{~s}, 3 \mathrm{H}), 1.31(\mathrm{~s}, 3 \mathrm{H}), 1.58-1.69(\mathrm{~m}, 2 \mathrm{H})$, $1.79(\mathrm{~s}, 3 \mathrm{H}), 2.05(\mathrm{~s}, 3 \mathrm{H}), 2.26(\mathrm{t}, J=7.2 \mathrm{~Hz}, 2 \mathrm{H}), 2.71(\mathrm{t}, J=6.3 \mathrm{~Hz}, 1 \mathrm{H}), 4.58(\mathrm{~d}, J=7.2 \mathrm{~Hz}, 2 \mathrm{H})$, $5.41(\mathrm{t}, J=7.2 \mathrm{~Hz}, 1 \mathrm{H})$.

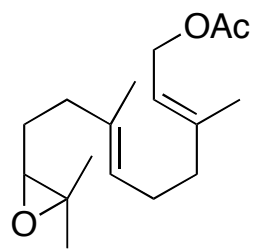

(2E,6E)-9-(3,3-Dimethyloxiran-2-yl)-3,7-dimethylnona-2,6-dienyl Acetate (26c) (Step b, Scheme 3): ${ }^{3}$ To a solution of $\mathbf{2 5 c}(9.14 \mathrm{~mL}, 30.0 \mathrm{mmol})$ in THF (700 mL) was added water $(530 \mathrm{~mL})$ at $0{ }^{\circ} \mathrm{C}$ until the solution became cloudy. NBS (recrystallized, $5.82 \mathrm{~g}, 32.7 \mathrm{mmol}$ ) was then added in small portions over $1 \mathrm{~h}$ and the reaction was allowed to stir for another hour at $0^{\circ} \mathrm{C}$. The THF was evaporated in vacuo at $\sim 5^{\circ} \mathrm{C}(2 \mathrm{~h})$ and the suspension was extracted with hexane- $\mathrm{Et}_{2} \mathrm{O}(4: 1 ; 4 \times 150$ $\mathrm{mL}$ ). The organic layers were dried over anhydrous $\mathrm{MgSO}_{4}$ and concentrated in vacuo to give a yellow oil as crude product. The oil was purified by flash chromatography $\left(\mathrm{SiO}_{2}\right.$, hexane-EtOAc $\left.=9: 1-4: 1\right)$ to give pure bromohydrine $(8.30 \mathrm{~g}, 23.0 \mathrm{mmol})$ as colorless oil in $77 \%$ yield. To a solution of bromohydrine in anhydrous $\mathrm{MeOH}$ was added $\mathrm{K}_{2} \mathrm{CO}_{3}(7.94 \mathrm{~g}, 57.4 \mathrm{mmol})$. The reaction was stirred for $2 \mathrm{~h}$ at room temperature. The solvent was removed in vacuo and the residue was taken up in $\mathrm{Et}_{2} \mathrm{O}$ (100 $\mathrm{mL}$ ) to form white suspension. The suspension was filtered through a plug of tightly packed celite which was washed with $\mathrm{Et}_{2} \mathrm{O}$ (3 times). The combined ether fractions were washed with brine, dried over anhydrous $\mathrm{Na}_{2} \mathrm{SO}_{4}$ and concentrated in vacuo to give epoxy alcohol as colorless oil. Epoxy alcohol was taken upon in pyridine $(15 \mathrm{~mL})$ and was treated with $\mathrm{Ac}_{2} \mathrm{O}(8.70 \mathrm{~mL}, 92.0 \mathrm{mmol})$ at room 
temperature for 2 hours. The mixture was poured into ice-water $(100 \mathrm{~mL})$ and the product was extracted with $\mathrm{Et}_{2} \mathrm{O}(2 \times 100 \mathrm{~mL})$. The organic layers were washed with citric acid $(20 \%, 2 \times 10 \mathrm{~mL})$, water $(30 \mathrm{~mL})$, saturated $\mathrm{NaHCO}_{3}(2 \times 30 \mathrm{~mL})$ and brine $(2 \times 30 \mathrm{~mL})$. The organics were dried over anhydrous $\mathrm{Na}_{2} \mathrm{SO}_{4}$ and concentrated in vacuo to give 26c as a colorless oil. TLC, $R_{\mathrm{f}}=0.41$ (hexaneEtOAc $=4: 1) ;{ }^{1} \mathrm{H}$ NMR $\left(\mathrm{CDCl}_{3}, 300 \mathrm{MHz}\right) \delta 1.26(\mathrm{~s}, 3 \mathrm{H}), 1.30(\mathrm{~s}, 3 \mathrm{H}), 1.58-1.66(\mathrm{~m}, 2 \mathrm{H}), 1.62(\mathrm{~s}, 3 \mathrm{H})$, $1.71(\mathrm{~s}, 3 \mathrm{H}), 2.04-2.21(\mathrm{~m}, 6 \mathrm{H}), 2.04(\mathrm{~s}, 3 \mathrm{H}), 2.69(\mathrm{dt}, J=2.7,6.3 \mathrm{~Hz}, 1 \mathrm{H}), 4.59(\mathrm{t}, J=6.9 \mathrm{~Hz}, 2 \mathrm{H})$, $5.16(\mathrm{dt}, J=1.2,5.4 \mathrm{~Hz}, 1 \mathrm{H}), 5.34(\mathrm{tq}, J=1.2,6.9 \mathrm{~Hz}, 1 \mathrm{H}) ;{ }^{13} \mathrm{C} \mathrm{NMR}\left(\mathrm{CDCl}_{3}, 75 \mathrm{MHz}\right) \delta 15.6,16.1$, $18.4,20.6,24.5,25.8,27.1,36.0,39.1,57.8,60.9,63.6,118.2,123.9,134.2,141.5,170.5$.

General Procedure for the Oxidative Cleavage of Epoxides 26 to Aldehydes 27 (Step c, Scheme 3): A solution of $\mathbf{2 6}(50.0 \mathrm{mmol})$ in $\mathrm{Et}_{2} \mathrm{O}(50 \mathrm{~mL})$ was added to a solution of $\mathrm{HIO}_{4} \cdot 2 \mathrm{H}_{2} \mathrm{O}(13.7$ $\mathrm{g}, 60.0 \mathrm{mmol})$ in THF $(250 \mathrm{~mL})$ at $0{ }^{\circ} \mathrm{C}$. The mixture was stirred for $30 \mathrm{~min}$. and then diluted with saturated $\mathrm{NaHCO}_{3}$. After stirring for $15 \mathrm{~min}$, the resulting solution was filtered through celite, and the filter cake was washed with $\mathrm{Et}_{2} \mathrm{O}$. The combined filtrates were extracted with $\mathrm{Et}_{2} \mathrm{O}$. The extracts were washed with water, saturated $\mathrm{NaHCO}_{3}$ and brine, dried over anhydrous $\mathrm{MgSO}_{4}$ and concentrated in vacuo.<smiles>CC(=O)OCC=C(C)CCC=O</smiles>

(E)-3-Methyl-6-oxohex-2-enyl acetate (27a, Scheme 4): ${ }^{\text {1a,4 }}$ The product was obtained as pale yellow oil and used for next step without further purification. TLC, $R_{\mathrm{f}}=0.31$ (hexane-EtOAc $=$ 4:1); ${ }^{1} \mathrm{H}$ NMR $\left(\mathrm{CDCl}_{3}, 300 \mathrm{MHz}\right) \delta 1.73(\mathrm{~s}, 3 \mathrm{H}), 2.06(\mathrm{~s}, 3 \mathrm{H}), 2.38(\mathrm{t}, J=7.5 \mathrm{~Hz}, 2 \mathrm{H}), 2.56-2.62(\mathrm{~m}$, $2 \mathrm{H}), 4.58(\mathrm{~d}, J=7.2 \mathrm{~Hz}, 2 \mathrm{H}), 5.33-5.29(\mathrm{~m}, 1 \mathrm{H}), 9.78(\mathrm{dd}, J=1.8,3.0 \mathrm{~Hz}, 1 \mathrm{H})$.<smiles>CC(=O)OC1=C(C)CCCO1</smiles>

(Z)-3-Methyl-6-oxohex-2-enyl Acetate (27b, Scheme 4): ${ }^{2} \quad 96 \%$ yield for 2 steps. $\mathrm{SiO}_{2}$ column chromatography (hexane-EtOAc $=10: 1$ ); colorless oil; TLC, $R_{\mathrm{f}}=0.32$ (hexane-EtOAc $=4: 1$ ); ${ }^{1} \mathrm{H} \mathrm{NMR}\left(\mathrm{CDCl}_{3}, 300 \mathrm{MHz}\right) \delta 1.76(\mathrm{~s}, 3 \mathrm{H}), 2.06(\mathrm{~s}, 3 \mathrm{H}), 2.43(\mathrm{t}, J=7.5 \mathrm{~Hz}, 2 \mathrm{H}), 2.54-2.59(\mathrm{~m}, 2 \mathrm{H})$, $4.58(\mathrm{~d}, J=7.2 \mathrm{~Hz}, 2 \mathrm{H}), 5.41(\mathrm{~m}, J=7.2 \mathrm{~Hz}, 1 \mathrm{H}), 9.79(\mathrm{t}, J=1.2 \mathrm{~Hz}, 1 \mathrm{H})$.

General Procedure for the Reduction of Aldehydes 27 to Alcohols 28 (Step d, Scheme 3): To a stirred solution of $27(50.0 \mathrm{mmol})$ in $\mathrm{EtOH}(150 \mathrm{~mL})$ was added $\mathrm{NaBH}_{4}(2.08 \mathrm{~g}, 55.0 \mathrm{mmol})$ slowly at $0{ }^{\circ} \mathrm{C}$ and the mixture stirred for $1 \mathrm{~h}$ at $0{ }^{\circ} \mathrm{C}$. Then $1 \mathrm{M} \mathrm{HCl}$ was added and the aqueous phase was extracted with EtOAc (3 times). The combined organic layers were washed with saturated $\mathrm{NaHCO}_{3}$, brine and water, dried over anhydrous $\mathrm{MgSO}_{4}$ and concentrated in vacuo.<smiles>CC(=O)OCC=C(C)CCCO</smiles>

(E)-6-Hydroxy-3-methylhex-2-enyl Acetate (28a): ${ }^{5}$ The product was obtained as a pale yellow oil and used for next step without further purification. TLC, $R_{\mathrm{f}}=0.10$ (hexane-EtOAc $\left.=4: 1\right)$; ${ }^{1} \mathrm{H}$ $\operatorname{NMR}\left(\mathrm{CDCl}_{3}, 300 \mathrm{MHz}\right) \delta 1.69-1.76(\mathrm{~m}, 2 \mathrm{H}), 1.72(\mathrm{~s}, 3 \mathrm{H}), 2.06(\mathrm{~s}, 3 \mathrm{H}), 2.06-2.16(\mathrm{~m}, 2 \mathrm{H}), 3.65(\mathrm{t}, J=$ $6.6 \mathrm{~Hz}, 2 \mathrm{H}), 4.58(\mathrm{~d}, J=7.2 \mathrm{~Hz}, 2 \mathrm{H}), 5.35-5.41(\mathrm{~m}, 1 \mathrm{H})$.<smiles>CC(=O)OC1CCCCO1</smiles>

(Z)-6-Hydroxy-3-methylhex-2-enyl Acetate (28b): ${ }^{6} \quad 96 \%$ yield. $\mathrm{SiO}_{2}$ column chromatography (hexane-EtOAc $=1: 1$ ); colorless oil; TLC, $R_{\mathrm{f}}=0.11$ (hexane-EtOAc $=4: 1$ ); ${ }^{1} \mathrm{H}$ NMR 
$\left(\mathrm{CDCl}_{3}, 300 \mathrm{MHz}\right) \delta 1.67$ (quintet, $\left.J=6.6 \mathrm{~Hz}, 2 \mathrm{H}\right), 1.75(\mathrm{~s}, 3 \mathrm{H}), 1.95$ (brs, OH), $2.04(\mathrm{~s}, 3 \mathrm{H}), 2.20(\mathrm{t}, J$ $=7.5 \mathrm{~Hz}, 2 \mathrm{H}), 3.60$ (brs, $J=2 \mathrm{H}), 4.58(\mathrm{~d}, J=7.2 \mathrm{~Hz}, 2 \mathrm{H}), 5.36(\mathrm{t}, J=7.2 \mathrm{~Hz}, 1 \mathrm{H})$.

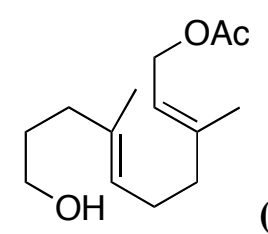

(2E,6E)-10-Hydroxy-3,7-dimethyldeca-2,6-dienyl Acetate (28c): ${ }^{7} 96 \%$ yield for 4 steps. $\mathrm{SiO}_{2}$ column chromatography (hexane-EtOAc $=1: 1$ ); pale yellow oil; TLC, $R_{\mathrm{f}}=0.11$ (hexaneEtOAc $=4: 1) ;{ }^{1} \mathrm{H} \mathrm{NMR}\left(\mathrm{CDCl}_{3}, 300 \mathrm{MHz}\right) \delta 1.61(\mathrm{~s}, 3 \mathrm{H}), 1.61-1.70(\mathrm{~m}, 2 \mathrm{H}), 1.71(\mathrm{~s}, 3 \mathrm{H}), 2.02-2.14$ $(\mathrm{m}, 6 \mathrm{H}), 2.06(\mathrm{~s}, 1 \mathrm{H}), 2.59(\mathrm{~s}, \mathrm{OH}), 3.59(\mathrm{t}, J=6.6 \mathrm{~Hz}, 2 \mathrm{H}), 4.58(\mathrm{t}, J=7.2 \mathrm{~Hz}, 2 \mathrm{H}), 5.13(\mathrm{dt}, J=1.2$, $7.2 \mathrm{~Hz}, 1 \mathrm{H}), 5.34$ (tq, $J=1.2,7.2 \mathrm{~Hz}, 1 \mathrm{H}) ;{ }^{13} \mathrm{C} \mathrm{NMR}\left(\mathrm{CDCl}_{3}, 75 \mathrm{MHz}\right) \delta 15.7,16.2,20.8,25.8,30.5$, $35.6,39.2,61.2,62.1,118.1,123.7,134.9,141.9,171.1$.

General Procedure for the Silylation of Alcohols 28 to Silyl Ethers 29 (Step e, Scheme 3): To a solution of 28 (22.1 mmol), imidazole (3.20 g, $24.3 \mathrm{mmol})$, in DMF (22 $\mathrm{mL})$ was added TBDPSCl $(6.30 \mathrm{~mL}, 24.3 \mathrm{mmol})$ at $0{ }^{\circ} \mathrm{C}$. After the mixture was stirred for over night, the reaction was quenched with water. The aqueous phase was extracted with $\mathrm{Et}_{2} \mathrm{O}$ (3 times), washed with brine, dried over anhydrous $\mathrm{MgSO}_{4}$ and concentrated in vacuo.

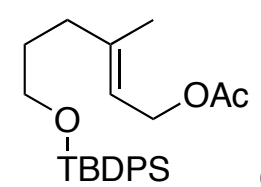

(E)-6-(tert-Butyldiphenylsilyloxy)-3-methylhex-2-enyl Acetate (29a): ${ }^{8}$ The product was obtained as a pale yellow oil and used for next step without further purification. TLC, $R_{\mathrm{f}}=0.50$ (hexane-EtOAc = 4:1); ${ }^{1} \mathrm{H}$ NMR $\left(\mathrm{CDCl}_{3}, 300 \mathrm{MHz}\right) \delta 1.05(\mathrm{~s}, 9 \mathrm{H}), 1,63-1,73(\mathrm{~m}, 2 \mathrm{H}), 1.67(\mathrm{~s}, 3 \mathrm{H})$, $2.05(\mathrm{~s}, 3 \mathrm{H}), 2.13(\mathrm{t}, J=7.5 \mathrm{~Hz}, 2 \mathrm{H}), 3.65(\mathrm{t}, J=6.3 \mathrm{~Hz}, 2 \mathrm{H}), 4.56(\mathrm{~d}, J=7.2 \mathrm{~Hz}, 2 \mathrm{H}), 5.33(\mathrm{tq}, J=1.2$, $7.2 \mathrm{~Hz}, 1 \mathrm{H}), 7.35-7.45(\mathrm{~m}, 6 \mathrm{H}), 7.65-7.73(\mathrm{~m}, 4 \mathrm{H})$.

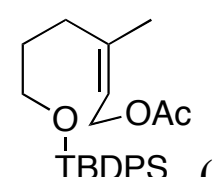

TBDPS (Z)-6-(tert-Butyldiphenylsilyloxy)-3-methylhex-2-enyl Acetate (29b): $98 \%$ yield; $\mathrm{SiO}_{2}$ column chromatography (hexane-EtOAc $=20: 1$ ); colorless oil; TLC, $R_{\mathrm{f}}=0.64$ (hexane-EtOAc $=4: 1$ ); IR (film) 3012, 2933, 2896, 2859, $1731(\mathrm{C}=\mathrm{O}), 1428,1381,1241,1111,1022 \mathrm{~cm}^{-1} ;{ }^{1} \mathrm{H} \mathrm{NMR}\left(\mathrm{CDCl}_{3}\right.$, $300 \mathrm{MHz}) \delta 1.05(\mathrm{~s}, 9 \mathrm{H}), 1.57-1.67(\mathrm{~m}, 2 \mathrm{H}), 1.73(\mathrm{~s}, 3 \mathrm{H}), 2.03(\mathrm{~s}, 3 \mathrm{H}), 2.18(\mathrm{t}, J=7.5 \mathrm{~Hz}, 2 \mathrm{H}), 3.65(\mathrm{t}$, $J=6.3 \mathrm{~Hz}, 2 \mathrm{H}), 4.56(\mathrm{t}, J=7.2 \mathrm{~Hz}, 2 \mathrm{H}), 5.35(\mathrm{t}, J=7.2 \mathrm{~Hz}, 1 \mathrm{H}), 7.35-7.45(\mathrm{~m}, 6 \mathrm{H}), 7.65-7.69(\mathrm{~m}$, $4 \mathrm{H}) ;{ }^{13} \mathrm{C} \mathrm{NMR}\left(\mathrm{CDCl}_{3}, 75 \mathrm{MHz}\right) \delta 18.9,20.6,23.1,26.6(3 \mathrm{C}), 28.0,30.7,60.6,63.0,119.2,127.4(4 \mathrm{C})$, 129.3 (2C), 133.5 (2C). 135.2 (4C), 141.8, 170.3 (C=O); HRMS (FAB) $m / z$ calcd for $\mathrm{C}_{25} \mathrm{H}_{34} \mathrm{O}_{3} \mathrm{SiNa}$ $(\mathrm{M}+\mathrm{Na}) 433.2175$, found 433.2175 .

General Procedure for the Alcoholysis of Acetates 29 to Alcohols 30 (Step f, Scheme 3): To a solution of $29(22.0 \mathrm{mmol})$ in anhydrous $\mathrm{MeOH}(22 \mathrm{~mL})$ was added $\mathrm{K}_{2} \mathrm{CO}_{3}(1.52 \mathrm{~g}, 11.0 \mathrm{mmol})$. The reaction was stirred for $1 \mathrm{~h}$ at room temperature. The $\mathrm{K}_{2} \mathrm{CO}_{3}$ was filtered off and the $\mathrm{MeOH}$ was evaporated. The residue was extracted with EtOAc (2 times), washed with brine, dried over anhydrous $\mathrm{MgSO}_{4}$ and concentrated in vacuo. The crude alcohols were purified by column chromatography on silica gel with hexane-EtOAc $=2: 1$ as eluents to afford pure $\mathbf{3 0}$. 


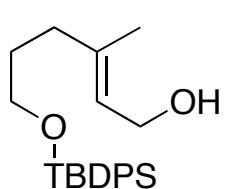

(E)-6-(tert-Butyldiphenylsilyloxy)-3-methylhex-2-en-1-ol (30a): ${ }^{8} 80 \%$ yield for 5 steps. Pale yellow oil; TLC, $R_{\mathrm{f}}=0.22$ (hexane-EtOAc $\left.=4: 1\right) ;{ }^{1} \mathrm{H}$ NMR $\left(\mathrm{CDCl}_{3}, 300 \mathrm{MHz}\right) \delta 1.05(\mathrm{~s}, 9 \mathrm{H})$, $1.64-1.75(\mathrm{~m}, 2 \mathrm{H}), 1.65(\mathrm{~s}, 3 \mathrm{H}), 2.08(\mathrm{t}, J=8.1 \mathrm{~Hz}, 2 \mathrm{H}), 3.65(\mathrm{t}, J=6.3 \mathrm{~Hz}, 2 \mathrm{H}), 4.12(\mathrm{t}, J=6.0 \mathrm{~Hz}$, $2 \mathrm{H}), 5.38(\mathrm{tq}, J=1.2,7.2 \mathrm{~Hz}, 1 \mathrm{H}), 7.35-7.45(\mathrm{~m}, 6 \mathrm{H}), 7.65-7.73(\mathrm{~m}, 4 \mathrm{H})$.

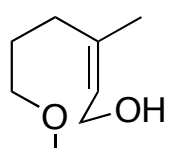

TBDPS (Z)-6-(tert-Butyldiphenylsilyloxy)-3-methylhex-2-en-1-ol (30b): $98 \%$ yield. Colorless oil; TLC, $R_{\mathrm{f}}=0.26$ (hexane-EtOAc $=4: 1$ ); IR (film) 3610, 3550-3200 (br), 3010, 2932, 2860, 1472, 1428, 1389, 1240, $1107 \mathrm{~cm}^{-1} ;{ }^{1} \mathrm{H} \mathrm{NMR}\left(\mathrm{CDCl}_{3}, 300 \mathrm{MHz}\right) \delta 1.06(\mathrm{~s}, 9 \mathrm{H}), 1.46(\mathrm{t}, J=5.4 \mathrm{~Hz}, 1 \mathrm{H}), 1.59$ (quintet, $J=6.0 \mathrm{~Hz}, 3 \mathrm{H}), 1.70(\mathrm{~s}, 3 \mathrm{H}), 2.19(\mathrm{t}, J=7.8 \mathrm{~Hz}, 2 \mathrm{H}), 3.65(\mathrm{t}, J=6.0 \mathrm{~Hz}, 2 \mathrm{H}), 4.11(\mathrm{t}, J=6.0$ $\mathrm{Hz}, 2 \mathrm{H}), 5.46(\mathrm{t}, J=6.0 \mathrm{~Hz}, 1 \mathrm{H}), 7.35-7.47(\mathrm{~m}, 6 \mathrm{H}), 7.64-7.69(\mathrm{~m}, 4 \mathrm{H}) ;{ }^{13} \mathrm{C} \mathrm{NMR}\left(\mathrm{CDCl}_{3}, 75 \mathrm{MHz}\right) \delta$ 19.0, 23.2, 26.8 (3C), 27.8, 30.7, 58.6, 63.1, 124.7, 127.5 (4C), 129.5 (2C), 133.6 (2C), 135.4 (4C), 139.2; HRMS (FAB) $\mathrm{m} / z$, calcd for $\mathrm{C}_{23} \mathrm{H}_{32} \mathrm{O}_{2} \mathrm{SiNa}(\mathrm{M}+\mathrm{Na}) 391.2069$, found 391,2057.

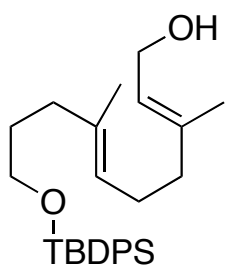

(2E,6E)-10-(tert-Butyldiphenylsilyloxy)-3,7-dimethyldeca-2,6-dien-1-ol $\quad(30 \mathrm{c}): \quad 95 \%$ yield for 2 steps. Pale yellow oil; TLC, $R_{\mathrm{f}}=0.22$ (hexane-EtOAc $\left.=4: 1\right) ;{ }^{1} \mathrm{H} \mathrm{NMR}\left(\mathrm{CDCl}_{3}, 300 \mathrm{MHz}\right) \delta$ $1.05(\mathrm{~s}, 9 \mathrm{H}), 1.46$ (brs, OH), $1.56(\mathrm{~s}, 3 \mathrm{H}), 1.60-1.70(\mathrm{~m}, 2 \mathrm{H}), 1.66(\mathrm{~s}, 3 \mathrm{H}), 1.98-2.12(\mathrm{~m}, 6 \mathrm{H}), 3.64(\mathrm{t}, J$ $=6.6 \mathrm{~Hz}, 2 \mathrm{H}), 4.12(\mathrm{~d}, J=6.9 \mathrm{~Hz}, 2 \mathrm{H}), 5.09(\mathrm{dt}, J=0.9,7.2 \mathrm{~Hz}, 1 \mathrm{H}), 5.39(\mathrm{dt}, J=1.2,7.2 \mathrm{~Hz}, 1 \mathrm{H})$, 7.34-7.44 (m, 6H), 7.66-7.70 (m, 4H); ${ }^{13} \mathrm{C} \mathrm{NMR}\left(\mathrm{CDCl}_{3}, 75 \mathrm{MHz}\right) \delta 15.9,16.2,19.1,26.2,26.8$ (3C), $30.8,35.7,39.4,59.2$, 63.4, 123.3, 123.8, 127.5 (4C), 129.4 (2C), 134.0 (2C), 134.9, 135.5 (4C), 139.5.

General Procedure for the Condensation of Alcohols 30 to Phosphonates 31 (Step g, Scheme 3): To a solution of $30(22.0 \mathrm{mmol})$ and $\mathrm{Et}_{3} \mathrm{~N}(4.60 \mathrm{~mL}, 33.0 \mathrm{mmol})$ in hexane $(30 \mathrm{~mL})$ was added $(\mathrm{EtO})_{2} \mathrm{POCl}(4.80 \mathrm{~mL}, 33.0 \mathrm{mmol})$ dropwise at $0{ }^{\circ} \mathrm{C}$. The mixture was warmed to room temperature and stirred for $24 \mathrm{~h}$. The reaction was quenched with saturated $\mathrm{NH}_{4} \mathrm{Cl}$, extracted with EtOAc (3 times), dried over anhydrous $\mathrm{MgSO}_{4}$ and concentrated in vacuo. The crude phosphonates were purified by column chromatography on silica gel to afford pure $\mathbf{3 1}$.

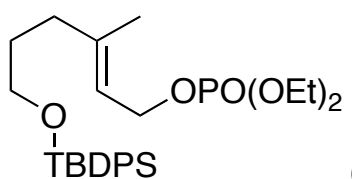

$90 \%$ yield. Column chromatography (hexane-EtOAc $=3: 1$ to $1: 1$ ); pale yellow oil; TLC, $R_{\mathrm{f}}=0.29$ (hexane-EtOAc = 2:1); IR (film) 3550-3250 (br), 3000, 2932, 2859, 1472, 1428, 1391, 1259, 1111, $1034 \mathrm{~cm}^{-1} ;{ }^{1} \mathrm{H}$ NMR $\left(\mathrm{CDCl}_{3}, 300 \mathrm{MHz}\right) \delta 1.06(\mathrm{~s}, 9 \mathrm{H}), 1.32(\mathrm{t}, J=7.2 \mathrm{~Hz}, 6 \mathrm{H}), 1.64-1.74(\mathrm{~m}, 2 \mathrm{H}), 1.69$ $(\mathrm{s}, 3 \mathrm{H}), 2.14(\mathrm{t}, J=7.8 \mathrm{~Hz}, 2 \mathrm{H}), 3.66(\mathrm{t}, J=6.3 \mathrm{~Hz}, 2 \mathrm{H}), 4.10(\mathrm{q}, J=7.2 \mathrm{~Hz}, 4 \mathrm{H}), 4.55(\mathrm{t}, J=7.5 \mathrm{~Hz}$, $2 \mathrm{H}), 5.41(\mathrm{t}, J=7.5 \mathrm{~Hz}, 1 \mathrm{H}), 7.34-7.44(\mathrm{~m}, 6 \mathrm{H}), 7.65-7.69(\mathrm{~m}, 4 \mathrm{H}) ;{ }^{13} \mathrm{C} \mathrm{NMR}\left(\mathrm{CDCl}_{3}, 75 \mathrm{MHz}\right) \delta 16.0$, 16.3, 19.0, 26.7, 30.3, 35.6, 63.1, 63.4, 63.5, 63.8, 63.9, 118.8, 127.5 (4C), 129.4 (2C), 133.7 (2C), 135.4 (4C), 142. 3; HRMS (FAB) $m / z$ calcd for $\mathrm{C}_{27} \mathrm{H}_{42} \mathrm{O}_{5} \mathrm{PSi}(\mathrm{M}+\mathrm{H})$ 505.2539, found 505.2544. 


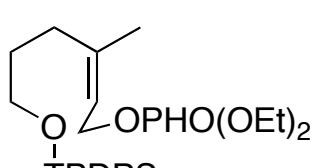

TBDPS

(Z)-6-(tert-Butyldiphenylsilyloxy)-3-methylhex-2-enyl Diethyl Phosphate (31b): $98 \%$ yield. Column chromatography (hexane-EtOAc $=2: 1$ ); colorless oil; TLC, $R_{\mathrm{f}}=0.29$ (hexaneEtOAc = 2:1); IR (film) 3500-3250 (br), 3000, 2959, 2860, 1472, 1428, 1391, 1259, 1111, $1032 \mathrm{~cm}^{-1}$; ${ }^{1} \mathrm{H} \mathrm{NMR}\left(\mathrm{CDCl}_{3}, 300 \mathrm{MHz}\right) \delta 1.05(\mathrm{~s}, 9 \mathrm{H}), 1.31(\mathrm{t}, J=7.5 \mathrm{~Hz}, 6 \mathrm{H}), 1.57-1.66(\mathrm{~m}, 2 \mathrm{H}), 1.73(\mathrm{~s}, 3 \mathrm{H})$, $2.18(\mathrm{t}, J=7.8 \mathrm{~Hz}, 2 \mathrm{H}), 3.64(\mathrm{t}, J=6.0 \mathrm{~Hz}, 2 \mathrm{H}), 4.08(\mathrm{q}, J=7.5 \mathrm{~Hz}, 4 \mathrm{H}), 4.54(\mathrm{t}, J=7.5 \mathrm{~Hz}, 2 \mathrm{H}), 5.40$ $(\mathrm{t}, J=6.9 \mathrm{~Hz}, 1 \mathrm{H}), 7.30-7.50(\mathrm{~m}, 6 \mathrm{H}), 7.64-7.70(\mathrm{~m}, 4 \mathrm{H}) ;{ }^{13} \mathrm{C} \mathrm{NMR}\left(\mathrm{CDCl}_{3}, 75 \mathrm{MHz}\right) \delta 16.0,19.0$, 23.3, 26.7 (3C), 28.2, 30.9, 63.2, 63.4 (2C), 63.5, 63.6, 119.8 (d, J = 26 Hz), 127.5 (4C), 129.5 (2C), 133.7 (2C), 135.4 (4C), 142.4; HRMS (FAB) $\mathrm{m} / z$ calcd for $\mathrm{C}_{27} \mathrm{H}_{42} \mathrm{O}_{5} \mathrm{PSi}(\mathrm{M}+\mathrm{H}) 505.2539$, found 505.2548 .

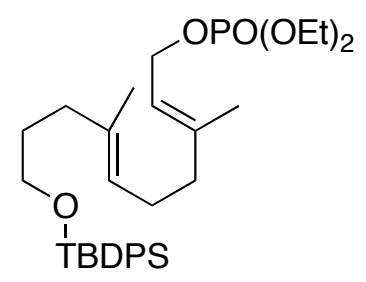

(2E,6E)-10-(tert-Butyldiphenylsilyloxy)-3,7-dimethyldeca-2,6-dienyl Diethyl Phosphate (31c): $91 \%$ yield. Column chromatography (hexane-EtOAc $=2: 1$ ); colorless oil; TLC, $R_{\mathrm{f}}=$ 0.25 (hexane-EtOAc = 2:1); ${ }^{1} \mathrm{H} \mathrm{NMR}\left(\mathrm{CDCl}_{3}, 300 \mathrm{MHz}\right) \delta 1.05(\mathrm{~s}, 9 \mathrm{H}), 1.33(\mathrm{t}, J=7.2 \mathrm{~Hz}, 6 \mathrm{H}), 1.56(\mathrm{~s}$, $3 \mathrm{H}), 1.56-1.70(\mathrm{~m}, 2 \mathrm{H}), 1.70(\mathrm{~s}, 3 \mathrm{H}), 1.99-2.12(\mathrm{~m}, 6 \mathrm{H}), 3.63(\mathrm{t}, J=6.6 \mathrm{~Hz}, 2 \mathrm{H}), 4.11$ (quintet, $J=7.2$ $\mathrm{Hz}, 4 \mathrm{H}), 4.56(\mathrm{t}, J=7.2 \mathrm{~Hz}, 2 \mathrm{H}), 5.08(\mathrm{dt}, J=1.2,7.2 \mathrm{~Hz}, 1 \mathrm{H}), 5.39(\mathrm{dt}, J=1.5,7.2 \mathrm{~Hz}, 1 \mathrm{H}), 7.35-7.46$ $(\mathrm{m}, 6 \mathrm{H}), 7.65-7.69(\mathrm{~m}, 4 \mathrm{H})$.

General Procedure for Butsugan Coupling of Phosphonates 31 with Arylmethyl Grignard Reagents (Step h, Scheme 3): ${ }^{9} \mathrm{Mg}(144 \mathrm{mg}, 6.0 \mathrm{mmol})$ was activated with stirring 2 days under $\mathrm{N}_{2}$ atmosphere and THF $(5 \mathrm{~mL})$ was added. The resulting mixture was cooled to $0{ }^{\circ} \mathrm{C}$ and arylmethyl chloride $(4.50 \mathrm{mmol})$ was added dropwise with stirring over period $30 \mathrm{~min}$. at $0{ }^{\circ} \mathrm{C}$. After stirring for 3 $\mathrm{h}$ used for next step. The solution of $31(3.0 \mathrm{mmol})$ in $\mathrm{THF}(6 \mathrm{~mL})$ was cooled to $0{ }^{\circ} \mathrm{C}$ under $\mathrm{N}_{2}$ atmosphere and Grignard reagent was added to this solution via cannula slowly. The mixture was warmed to room temperature and stirred for $12 \mathrm{~h}$. After the reaction was completed (TLC), quenched with saturated $\mathrm{NH}_{4} \mathrm{Cl}$, the aqueous phase was extracted with $\mathrm{Et}_{2} \mathrm{O}$ (twice), the comined organic layers was dried over anhydrous $\mathrm{MgSO}_{4}$ and concentrated in vacuo. The crude product was dissolved in THF $(30 \mathrm{~mL})$ and to this solution was added TBAF (1.0 $M$ in THF, TCI, $6.0 \mathrm{~mL}, 6.0 \mathrm{mmol})$. After the resulting mixture was stirred for $6 \mathrm{~h}$, the reaction was quenched with water and the aqueous layer was extracted with EtOAc (3 times). The combined organic layers were dried over anhydrous $\mathrm{MgSO}_{4}$ and concentrated in vacuo. The residue was purified by column chromatography on silica gel to afford pure 32.<smiles>CC(=CCCc1ccc(F)cc1)CCCO</smiles>

(E)-7-(4-Fluorophenyl)-4-methylhept-4-en-1-ol (32aa): $\quad>99 \%$ yield. Column chromatography (hexane-EtOAc $=3: 1$ ); pale yellow oil; $\mathrm{TLC}, R_{\mathrm{f}}=0.13$ (hexane-EtOAc $=4: 1$ ); ${ }^{1} \mathrm{H}$ $\operatorname{NMR}\left(\mathrm{CDCl}_{3}, 300 \mathrm{MHz}\right) \delta 1.39$ (brs, OH), 1.54 (s, 3H), 1.60-1.69 (m, 2H), 2.05 (t, $\left.J=7.5 \mathrm{~Hz}, 2 \mathrm{H}\right)$, $2.29(\mathrm{t}, J=7.5 \mathrm{~Hz}, 2 \mathrm{H}), 2.61(\mathrm{t}, J=7.5 \mathrm{~Hz}, 2 \mathrm{H}), 3.60(\mathrm{dd}, J=6.0,9.9 \mathrm{~Hz}, 2 \mathrm{H}), 5.19(\mathrm{tq}, J=1.5,7.2 \mathrm{~Hz}$, 1H), 6.91-6.99 (m, 2H), 7.09-7.16 (m, 2H); ${ }^{13} \mathrm{C} \mathrm{NMR}\left(\mathrm{CDCl}_{3}, 75 \mathrm{MHz}\right) \delta 15.6,29.8,30.6,35.0,35.7$, 
62.2, 114.6, 114.8, 123.4, 129.5, 129.6, 135.4, 137.6, $161.0\left(\mathrm{~d}, J_{\mathrm{CF}}=242 \mathrm{~Hz}\right) ;{ }^{19} \mathrm{~F} \mathrm{NMR}\left(\mathrm{CDCl}_{3}, 282\right.$ $\mathrm{MHz}) \delta-118.5\left(\mathrm{dd}, J_{\mathrm{CF}}=9.0,15.0 \mathrm{~Hz}\right)$.<smiles>CC(=CCCc1ccccc1)CCCO</smiles>

$(\boldsymbol{E})$-4-Methyl-7-phenylhept-4-en-1-ol (32ab) ${ }^{10} \quad 97 \%$ yield. Column chromatography (hexane-EtOAc $=5: 1$ ); colorless oil; TLC, $R_{\mathrm{f}}=0.15$ (hexane-EtOAc $\left.=4: 1\right) ;{ }^{1} \mathrm{H} \mathrm{NMR}\left(\mathrm{CDCl}_{3}, 300\right.$ $\mathrm{MHz}) \delta 1,25(\mathrm{t}, J=5.1 \mathrm{~Hz}, \mathrm{OH}), 1.56(\mathrm{~s}, 3 \mathrm{H}), 1.66(\mathrm{q}, J=6.6 \mathrm{~Hz}, 2 \mathrm{H}), 2.05(\mathrm{t}, J=7.2 \mathrm{~Hz}, 2 \mathrm{H}), 2.33(\mathrm{q}$, $J=7.5 \mathrm{~Hz}, 2 \mathrm{H}), 2.64(\mathrm{t}, J=7.8 \mathrm{~Hz}, 2 \mathrm{H}), 3.60(\mathrm{dd}, J=6.3,11.7 \mathrm{~Hz}, 2 \mathrm{H}), 5.22(\mathrm{dt}, J=1.5,7.2 \mathrm{~Hz}, 1 \mathrm{H})$, 7.18-7.20 (m, 3H), 7.26-7.30 (m, 2H).<smiles>CC1=CCCc2ccc(C)cc21</smiles>

(E)-4-Methyl-7-p-tolylhept-4-en-1-ol (32ac): 95\% yield. Column chromatography (hexane-EtOAc = 3:1); colorless oil; TLC, $R_{\mathrm{f}}=0.19$ (hexane-EtOAc = 4:1); IR (film) 3619, 3550-3200 (br), 3010, 2939, 1514, 1449, 1385, 1054, 1008, $814 \mathrm{~cm}^{-1}$; ${ }^{1} \mathrm{H}$ NMR $\left(\mathrm{CDCl}_{3}, 300 \mathrm{MHz}\right) \delta 1.36$ (brs, OH), $1.57(\mathrm{~s}, 3 \mathrm{H}), 1.65$ (quintet, $J=6.9 \mathrm{~Hz}, 2 \mathrm{H}), 2.05(\mathrm{t}, J=7.5 \mathrm{~Hz}, 2 \mathrm{H}), 2.24-2.35(\mathrm{~m}, 2 \mathrm{H}), 2.32(\mathrm{~s}, 3 \mathrm{H})$, $2.60(\mathrm{t}, J=7.8 \mathrm{~Hz}, 2 \mathrm{H}), 3.60(\mathrm{t}, J=6.6 \mathrm{~Hz}, 2 \mathrm{H}), 5.22(\mathrm{tq}, J=1.2,7.2 \mathrm{~Hz}, 1 \mathrm{H}), 7.08(\mathrm{~s}, 4 \mathrm{H}) ;{ }^{13} \mathrm{C} \mathrm{NMR}$ $\left(\mathrm{CDCl}_{3}, 75 \mathrm{MHz}\right) \delta 15.6,20.7,29.8,35.4,35.6,62.0,123.8,128.0$ (2C), 128.7 (2C), 134.9, 138.9; HRMS (FAB) $m / z$ calcd for $\mathrm{C}_{15} \mathrm{H}_{23} \mathrm{O}(\mathrm{M}+\mathrm{H}) 219.1749$, found 219.1739.<smiles>CC(=CCCc1cccc(C)c1)CCCO</smiles>

(E)-4-Methyl-7-m-tolylhept-4-en-1-ol (32ad): 94\% yield. Column chromatography (hexane-EtOAc $=4: 1$ ); pale yellow oil; TLC, $R_{\mathrm{f}}=0.24$ (hexane-EtOAc $=4: 1$ ); IR (film) 3627, 3500 3300 (br), 3011, 2940, 2850, 2360, 1456, 1054, $1010 \mathrm{~cm}^{-1} ;{ }^{1} \mathrm{H} \mathrm{NMR}\left(\mathrm{CDCl}_{3}, 300 \mathrm{MHz}\right) \delta 1.55$ (s, 3H), 1.62 (quintet, $J=7.2 \mathrm{~Hz}, 2 \mathrm{H}), 2.02(\mathrm{t}, J=7.5 \mathrm{~Hz}, 2 \mathrm{H}), 2.24-2.31(\mathrm{~m}, 2 \mathrm{H}), 2.30(\mathrm{~s}, 3 \mathrm{H}), 2.48(\mathrm{brs}, \mathrm{OH})$, $2.58(\mathrm{t}, J=7.8 \mathrm{~Hz}, 2 \mathrm{H}), 3.54(\mathrm{t}, J=6.6 \mathrm{~Hz}, 2 \mathrm{H}), 5.20(\mathrm{brt}, J=7.2 \mathrm{~Hz}, 1 \mathrm{H}), 6.95-6.98(\mathrm{~m}, 3 \mathrm{H}), 7.14(\mathrm{t}, J$ $=7.5 \mathrm{~Hz}, 1 \mathrm{H}) ;{ }^{13} \mathrm{C} \mathrm{NMR}\left(\mathrm{CDCl}_{3}, 75 \mathrm{MHz}\right) \delta 15.7,21.2,29.8,30.6,35.7,35.8,62.3,123.9,125.3,126.3$, 128.0, 129.1, 135.1, 137.5, 142.0; HRMS (FAB) $m / z$ calcd for $\mathrm{C}_{15} \mathrm{H}_{23} \mathrm{O}(\mathrm{M}+\mathrm{H})$ 219.1749, found 219.1759.<smiles>CCCC1=CCCc2cc(C(C)C)ccc21</smiles>

(E)-7-(3-Isopropylphenyl)-4-methylhept-4-en-1-ol (32ae): 95\% yield. Column chromatography (hexane-EtOAc $=5: 1)$; colorless oil; TLC, $R_{\mathrm{f}}=0.25$ (hexane-EtOAc $\left.=4: 1\right)$; IR $($ film) 3623, 3600-3250 (br), 3009, 2962, 2938, 2871, 1604, 1.445, $1051 \mathrm{~cm}^{-1} ;{ }^{1} \mathrm{H}$ NMR $\left(\mathrm{CDCl}_{3}, 300 \mathrm{MHz}\right) \delta$ $1.24(\mathrm{~d}, J=6.9 \mathrm{~Hz}, 6 \mathrm{H}), 1.30(\mathrm{t}, J=5.4 \mathrm{~Hz}, \mathrm{OH}), 1.57(\mathrm{~s}, 3 \mathrm{H}), 1.65$ (quintet, $J=7.5 \mathrm{~Hz}, 2 \mathrm{H}), 2.06(\mathrm{t}, J$ $=7.5 \mathrm{~Hz}, 2 \mathrm{H}), 2.31(\mathrm{q}, J=7.5 \mathrm{~Hz}, 2 \mathrm{H}), 2.63(\mathrm{t}, J=7.8 \mathrm{~Hz}, 2 \mathrm{H}), 2.80-2.94(\mathrm{~m}, 1 \mathrm{H}), 3.60(\mathrm{dd}, J=6.0$, $9.0 \mathrm{~Hz}, 2 \mathrm{H}), 5.23(\mathrm{t}, J=7.2 \mathrm{~Hz}, 1 \mathrm{H}), 6.99-7.06(\mathrm{~m}, 3 \mathrm{H}), 7.21(\mathrm{t}, J=7.8 \mathrm{~Hz}, 1 \mathrm{H}) ;{ }^{13} \mathrm{C} \mathrm{NMR}\left(\mathrm{CDCl}_{3}, 75\right.$ $\mathrm{MHz}) \delta 15.7,23.9$ (2C), 29.8, 30.6, 33.9, 35.7, 35.9, 62.1, 123.5, 123.9, 125.7, 125.4, 128.0, 135.0, 142.0, 148.5; HRMS (FAB) $\mathrm{m} / z$, calcd for $\mathrm{C}_{17} \mathrm{H}_{26} \mathrm{ONa}(\mathrm{M}+\mathrm{Na}) 269.1881$, found 269.1873. 
<smiles>Cc1ccc(CC2CCCOC2)cc1</smiles>

(Z)-4-Methyl-7-p-tolylhept-4-en-1-ol (32ba): $94 \%$ yield. Column chromatography (hexane-EtOAc $=5: 1)$; colorless oil; TLC, $R_{\mathrm{f}}=0.21$ (hexane-EtOAc $\left.=4: 1\right) ;{ }^{1} \mathrm{H} \mathrm{NMR}\left(\mathrm{CDCl}_{3}, 300\right.$ $\mathrm{MHz}) \delta 1.23(\mathrm{t}, J=5.4 \mathrm{~Hz}, \mathrm{OH}), 1.54-1.63(\mathrm{~m}, 2 \mathrm{H}), 1.70(\mathrm{~s}, 3 \mathrm{H}), 2.07(\mathrm{t}, J=7.5 \mathrm{~Hz}, 2 \mathrm{H}), 2.26-2.35(\mathrm{~m}$, 2H), $2.32(\mathrm{~s}, 3 \mathrm{H}), 2.60(\mathrm{t}, J=7.5 \mathrm{~Hz}, 2 \mathrm{H}), 3.58(\mathrm{q}, J=6.3 \mathrm{~Hz}, 2 \mathrm{H}), 5.21(\mathrm{t}, J=7.2 \mathrm{~Hz}, 1 \mathrm{H}), 7.09(\mathrm{~s}, 4 \mathrm{H})$.<smiles>CC1=CCCCO1</smiles>

(Z)-4-Methyl-7-m-tolylhept-4-en-1-ol (32bb): 94\% yield. Column chromatography (hexane-EtOAc = 5:1); colorless oil; TLC, $R_{\mathrm{f}}=0.33$ (hexane-EtOAc $=4: 1$ ); IR (film) 3628, 3650-3250 (br), 3012, 2932, 2860, 1607, 1456, 1378, 1041, $1009 \mathrm{~cm}^{-1} ;{ }^{1} \mathrm{H}$ NMR $\left(\mathrm{CDCl}_{3}, 300 \mathrm{MHz}\right) \delta 1.25(\mathrm{t}, J=$ $5.4 \mathrm{~Hz}, \mathrm{OH}), 1.58(\mathrm{dt}, J=6.6,13.2 \mathrm{~Hz}, 2 \mathrm{H}), 1.70(\mathrm{~s}, 3 \mathrm{H}), 2.07(\mathrm{t}, J=7.8 \mathrm{~Hz}, 2 \mathrm{H}), 2.26-2.33(\mathrm{~m}, 2 \mathrm{H})$, $2.33(\mathrm{~s}, 3 \mathrm{H}), 2.60(\mathrm{t}, J=7.8 \mathrm{~Hz}, 2 \mathrm{H}), 3.58(\mathrm{dd}, J=3.3,11.7 \mathrm{~Hz}, 2 \mathrm{H}), 5.22(\mathrm{t}, J=6.9 \mathrm{~Hz}, 1 \mathrm{H}), 6.97-7.00$ (m, 3H), 7.15-7.20 (m, 1H); ${ }^{13} \mathrm{C} \mathrm{NMR}\left(\mathrm{CDCl}_{3}, 75 \mathrm{MHz}\right) \delta 21.3,23.2,27.9,29.8,30.6,36.1,62.5,124.8$, 125.3, 126.3, 128.0, 129.1, 135.2, 137.6, 142.1; HRMS (FAB) $\mathrm{m} / z$ calcd for $\mathrm{C}_{15} \mathrm{H}_{23} \mathrm{O}(\mathrm{M}+\mathrm{H}) 219.1749$, found 219.1740 .<smiles>CC1=CCCC(Cc2ccc(C)cc2)=C1</smiles>

(4E,8E)-4,8-Dimethyl-11-p-tolylundeca-4,8-dien-1-ol $\quad$ (32ca): $99 \%$ yield. Column chromatography (hexane-EtOAc $=5: 1$ ); colorless oil; TLC, $R_{\mathrm{f}}=0.28$ (hexane-EtOAc $=4: 1$ ); IR (film) 3619, 3650-3200 (br), 3010, 2925, 2858, 1514, 1449, 1384, $1054 \mathrm{~cm}^{-1}$; ${ }^{1} \mathrm{H}$ NMR $\left(\mathrm{CDCl}_{3}, 300\right.$ $\mathrm{MHz}) \delta 1.56(\mathrm{~s}, 3 \mathrm{H}), 1.60(\mathrm{~s}, 3 \mathrm{H}), 1.60-1.69(\mathrm{~m}, 2 \mathrm{H}), 1.72(\mathrm{brs}, \mathrm{OH}), 1.96-2.11(\mathrm{~m}, 6 \mathrm{H}), 2.24-2.34(\mathrm{~m}$, $2 \mathrm{H}), 2.31(\mathrm{~s}, 3 \mathrm{H}), 2.60(\mathrm{dd}, J=6.7,9.0 \mathrm{~Hz}, 2 \mathrm{H}), 3.59(\mathrm{t}, J=6.6 \mathrm{~Hz}, 2 \mathrm{H}), 5.12-5.21(\mathrm{~m}, J=1.2,6.6 \mathrm{~Hz}$, 2H), $7.08(\mathrm{~s}, 4 \mathrm{H}) ;{ }^{13} \mathrm{C} \mathrm{NMR}\left(\mathrm{CDCl}_{3}, 75 \mathrm{MHz}\right) \delta 15.8,15.9,20.9,26.4,30.0,30.6,35.6,35.9,39.5,62.6$, 123.8, 124.6, 128.2 (2C), 128.8 (2C), 134.5, 135.0, 135.4, 139.2; HRMS (FAB) $\mathrm{m} / z$ calcd for $\mathrm{C}_{20} \mathrm{H}_{30} \mathrm{ONa}(\mathrm{M}+\mathrm{Na}) 309.2194$, found 309.2197.<smiles></smiles>

(4E,8E)-4,8-Dimethyl-11-m-tolylundeca-4,8-dien-1-ol (32cb): 97\% yield. Column chromatography (hexane-EtOAc $=10: 1)$; colorless oil; TLC, $R_{\mathrm{f}}=0.26$ (hexane-EtOAc $\left.=4: 1\right) ;$ IR (film) 3620, 3650-3200 (br), 3010, 2926, 2858, 1607, 1449, 1384, $1055 \mathrm{~cm}^{-1} ;{ }^{1} \mathrm{H} \mathrm{NMR}\left(\mathrm{CDCl}_{3}, 300 \mathrm{MHz}\right) \delta$ 1.37 (brs, OH), 1.57 (s, 3H), $1.62(\mathrm{~s}, 3 \mathrm{H}), 1.62-1.72(\mathrm{~m}, 2 \mathrm{H}), 1.97-2.12(\mathrm{~m}, 6 \mathrm{H}), 2.26-2.34(\mathrm{~m}, 2 \mathrm{H})$, $2.34(\mathrm{~s}, 3 \mathrm{H}), 2.60(\mathrm{t}, J=7.5 \mathrm{~Hz}, 2 \mathrm{H}), 3.63(\mathrm{t}, J=6.6 \mathrm{~Hz}, 2 \mathrm{H}), 5.13-5.22(\mathrm{~m}, 2 \mathrm{H}), 6.99-7.02(\mathrm{~m}, 3 \mathrm{H})$, $7.18(\mathrm{dt}, J=1.5,7.5 \mathrm{~Hz}, 1 \mathrm{H}) ;{ }^{13} \mathrm{C} \mathrm{NMR}\left(\mathrm{CDCl}_{3}, 75 \mathrm{MHz}\right) \delta 15.8,15.9,21.4,26.5,30.0,30.6,35.9,36.0$, 39.6, 62.6, 123.8, 124.6, 125.4, 126.3, 128.1, 129.2, 134.6, 135.5, 137.6, 142.3; HRMS (EI) $\mathrm{m} / z$ calcd for $\mathrm{C}_{20} \mathrm{H}_{30} \mathrm{O}(\mathrm{M}) 286.2297$, found 286.2301.

General Procedure for the Carbon Chain Homologation from Alcohols 32 to Nitriles 33 
(Step I, Scheme 3): To a solution of $32(5.86 \mathrm{mmol})$ in $\mathrm{CH}_{2} \mathrm{Cl}_{2}$ was added $\mathrm{Et}_{3} \mathrm{~N}(2.45 \mathrm{~mL}, 17.6 \mathrm{mmol})$ and $\mathrm{MsCl}(680 \mu \mathrm{L}, 8.79 \mathrm{mmol})$ dropwise at $0{ }^{\circ} \mathrm{C}$. After the resulting mixture was stirred for $1 \mathrm{~h}$ at $0{ }^{\circ} \mathrm{C}$, the mixture was allowed to warm to room temperature and stirred for $30 \mathrm{~min}$. Then the solvent was evaporated in vacuo. The residue was taken in the EtOAc-water (1:1) and the aqueous layer was extracted with EtOAc (3 times). The combined organic layers were dried over anhydrous $\mathrm{Na}_{2} \mathrm{SO}_{4}$ and concentrated in vacuo. The crude mesylate was dissolved in $\mathrm{CH}_{3} \mathrm{CN}(30 \mathrm{~mL})$ and was added 18-crown$6(4.65 \mathrm{~g}, 17.6 \mathrm{mmol})$ and $\mathrm{KCN}(1.14 \mathrm{~g}, 17.6 \mathrm{mmol})$. The resulting mixture was heated to reflux and stirred for $6 \mathrm{~h}$. After the reaction was quenched with water, the aqueous layer was extracted with $\mathrm{Et}_{2} \mathrm{O}$ (twice), the combined organic layers were dried over anhydrous $\mathrm{MgSO}_{4}$ and concentrated in vacuo.<smiles>CCCCC1=CCCc2ccc(F)cc21</smiles>

(E)-8-(4-Fluorophenyl)-5-methyloct-5-enenitrile (33aa): The product was obtained as a pale yellow oil and used for next step without further purification. TLC, $R_{\mathrm{f}}=0.44$ (hexane-EtOAc $=$ 4:1); IR (film) 3022, 2927, 2859, 2249, 1603, 1509, 1456, 1223, 1157, 830; ${ }^{1} \mathrm{H}$ NMR $\left(\mathrm{CDCl}_{3}, 300\right.$ $\mathrm{MHz}) \delta 1.50(\mathrm{~s}, 3 \mathrm{H}), 1.72$ (quintet, $J=7.2 \mathrm{~Hz}, 2 \mathrm{H}), 2.11(\mathrm{t}, J=7.2 \mathrm{~Hz}, 2 \mathrm{H}), 2.20(\mathrm{t}, J=7.2 \mathrm{~Hz}, 2 \mathrm{H})$, $2.30(\mathrm{q}, J=7.2 \mathrm{~Hz}, 2 \mathrm{H}), 2.63(\mathrm{t}, J=7.5 \mathrm{~Hz}, 2 \mathrm{H}), 5.21(\mathrm{tq}, J=1.2,7.2 \mathrm{~Hz}, 1 \mathrm{H}), 6.92-7.00(\mathrm{~m}, 2 \mathrm{H})$, 7.09-7.16 (m, 2H); ${ }^{13} \mathrm{C} \mathrm{NMR}\left(\mathrm{CDCl}_{3}, 75 \mathrm{MHz}\right) \delta 15.1,15.7,22.9,29.6,34.6,37.8,114.4,114.7,119.8$, $125.2,129.5,129.6,133.1,137.4,160.4\left(\mathrm{~d}, J_{\mathrm{C}-\mathrm{F}}=242\right) ;{ }^{19} \mathrm{~F} \mathrm{NMR}\left(\mathrm{CDCl}_{3}, 282 \mathrm{MHz}\right) \delta-118.3\left(\mathrm{dd}, J_{\mathrm{CF}}=\right.$ 9.0, $18 \mathrm{~Hz}$ ); HRMS (FAB) $m / z$ calcd for $\mathrm{C}_{15} \mathrm{H}_{19} \mathrm{NF}(\mathrm{M}+\mathrm{H})$ 232.1502, found 232.1490.

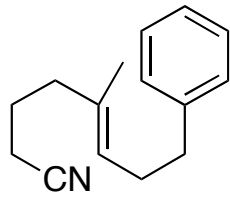

(E)-5-Methyl-8-phenyloct-5-enenitrile (33ab): The product was obtained as a pale yellow oil and used for next step without further purification. TLC, $R_{\mathrm{f}}=0.41$ (hexane-EtOAc $=4: 1$ ); ${ }^{1} \mathrm{H}$ $\operatorname{NMR}\left(\mathrm{CDCl}_{3}, 300 \mathrm{MHz}\right) \delta 1.51(\mathrm{~s}, 3 \mathrm{H}), 1.72$ (quintet, $\left.J=7.2 \mathrm{~Hz}, 2 \mathrm{H}\right), 2.11(\mathrm{t}, J=7.2 \mathrm{~Hz}, 2 \mathrm{H}), 2.17(\mathrm{t}$, $J=7.2 \mathrm{~Hz}, 2 \mathrm{H}), 2.33(\mathrm{t}, J=7.2 \mathrm{~Hz}, 2 \mathrm{H}), 2.67(\mathrm{t}, J=7.5 \mathrm{~Hz}, 2 \mathrm{H}), 5.22(\mathrm{tq}, J=1.2,7.2 \mathrm{~Hz}, 1 \mathrm{H}), 7.17-$ $7.21(\mathrm{~m}, 3 \mathrm{H}), 7.26-7.28(\mathrm{~m}, 2 \mathrm{H})$.<smiles>CCCCCCc1ccc(C)cc1</smiles>

(E)-5-Methyl-8-p-tolyloct-5-enenitrile (33ac): 95\% yield. $\mathrm{SiO}_{2}$ column chromatography (hexane-EtOAc $=10: 1)$; colorless oil; TLC, $R_{\mathrm{f}}=0.46$ (hexane-EtOAc $\left.=4: 1\right)$; IR $($ film) 3019, 2925, 2858, 2249, 1515, 1455, 1424, 1384, 1110, $812 \mathrm{~cm}^{-1} ;{ }^{1} \mathrm{H} \mathrm{NMR}\left(\mathrm{CDCl}_{3}, 300 \mathrm{MHz}\right) \delta 1.52(\mathrm{~s}, 3 \mathrm{H}), 1.72$ (quintet, $J=7.2 \mathrm{~Hz}, 2 \mathrm{H}), 2.10(\mathrm{t}, J=7.2 \mathrm{~Hz}, 2 \mathrm{H}), 2.16(\mathrm{t}, J=7.2 \mathrm{~Hz}, 2 \mathrm{H}), 2.27-2.34(\mathrm{~m}, 2 \mathrm{H}), 2.32(\mathrm{~s}$, $3 \mathrm{H}), 2.62(\mathrm{t}, J=7.5 \mathrm{~Hz}, 2 \mathrm{H}), 5.22(\mathrm{tq}, J=1.2,7.2 \mathrm{~Hz}, 1 \mathrm{H}), 7.05-7.11(\mathrm{~m}, 4 \mathrm{H}) ;{ }^{13} \mathrm{C} \mathrm{NMR}\left(\mathrm{CDCl}_{3}, 75\right.$ $\mathrm{MHz}) \delta 15.0,15.4,20.5,22.8,29.4,34.9,37.6,119.3,125.5,127.9$ (2C), 128.5 (2C), 132.5, 134.6, 138.4; HRMS (FAB) $m / z$ calcd for $\mathrm{C}_{16} \mathrm{H}_{22} \mathrm{~N}(\mathrm{M}+\mathrm{H}) 228.1752$, found 228.1755.<smiles>Cc1cccc(CCCC(C)CCCC#N)c1</smiles>

(E)-5-Methyl-8-m-tolyloct-5-enenitrile (33ad): $\quad 96 \%$ yield. $\quad \mathrm{SiO}_{2} \quad$ column chromatography (hexane-EtOAc $=10: 1)$; colorless oil; TLC, $R_{\mathrm{f}}=0.56($ hexane-EtOAc $=4: 1) ;$ IR $($ film $)$ 
3020, 3012, 2925, 2858, 2249, 1608, 1456, $1096 \mathrm{~cm}^{-1}$; ${ }^{1} \mathrm{H}$ NMR (CDCl, $\left.300 \mathrm{MHz}\right) \delta 1.48(\mathrm{~s}, 3 \mathrm{H}), 1.62$ (quintet, $J=7.2 \mathrm{~Hz}, 2 \mathrm{H}), 2.05(\mathrm{t}, J=7.2 \mathrm{~Hz}, 2 \mathrm{H}), 2.25-2.32(\mathrm{~m}, 4 \mathrm{H}), 2.30(\mathrm{~s}, 3 \mathrm{H}), 2.59(\mathrm{t}, J=7.2 \mathrm{~Hz}$, $2 \mathrm{H}), 5.19(\mathrm{tq}, J=1.2,7.2 \mathrm{~Hz}, 1 \mathrm{H}), 6.93-6.96(\mathrm{~m}, 3 \mathrm{H}), 7.13(\mathrm{t}, J=7.5 \mathrm{~Hz}, 1 \mathrm{H}) ;{ }^{13} \mathrm{C}$ NMR $\left(\mathrm{CDCl}_{3}, 75\right.$ MHz) $\delta$ 15.1, 15.5, 21.0, 22.8, 29.5, 35.4, 37.7, 119.4, 125.1, 125.6, 126.1, 127.8, 128.9, 132.6, 137.3, 141.5; HRMS (FAB) $\mathrm{m} / z$ calcd for $\mathrm{C}_{16} \mathrm{H}_{22} \mathrm{~N}(\mathrm{M}+\mathrm{H}) 228.1752$, found 228.1761 .<smiles>CCCCCCc1cccc(CCCCC)c1</smiles>

(E)-8-(3-Isopropylphenyl)-5-methyloct-5-enenitrile (33ae): $95 \%$ yield. $\quad \mathrm{SiO}_{2}$ column chromatography (hexane-EtOAc $=20: 1$ ); colorless oil; TLC, $R_{\mathrm{f}}=0.43$ (hexane-EtOAc $=4: 1$ ); IR (film) 2962, 2926, 2870, 2249, 1604, 1487, $1458 \mathrm{~cm}^{-1} ;{ }^{1} \mathrm{H}$ NMR $\left(\mathrm{CDCl}_{3}, 300 \mathrm{MHz}\right) \delta 11.24$ (d, $J=$ $6.9 \mathrm{~Hz}, 6 \mathrm{H}), 1.52(\mathrm{~s}, 3 \mathrm{H}), 1.72$ (quintet, $J=7.5 \mathrm{~Hz}, 2 \mathrm{H}), 2.08-2.19(\mathrm{~m}, 4 \mathrm{H}), 2.32$ (q, $J=7.5 \mathrm{~Hz}, 2 \mathrm{H})$, $2.64(\mathrm{t}, J=7.8 \mathrm{~Hz}, 2 \mathrm{H}), 2.80-2.94(\mathrm{~m}, 1 \mathrm{H}), 5.23(\mathrm{dt}, J=1.2,6.9 \mathrm{~Hz}, 1 \mathrm{H}), 6.99-7.07(\mathrm{~m}, 3 \mathrm{H}), 7.21(\mathrm{t}, J$ $=7.5 \mathrm{~Hz}, 1 \mathrm{H}) ;{ }^{13} \mathrm{C} \mathrm{NMR}\left(\mathrm{CDCl}_{3}, 75 \mathrm{MHz}\right) \delta 15.2,15.7,23.0,23.8(2 \mathrm{C}), 29.5,33.8,35.6,37.8,119.5$, $123.5,125.7,125.8,126.3,127.9,132.7,141.6,148.5$; HRMS (FAB) $\mathrm{m} / z$ calcd for $\mathrm{C}_{18} \mathrm{H}_{25} \mathrm{NNa}(\mathrm{M}+\mathrm{Na})$ 278.1885 , found 278.1884 .<smiles>Cc1ccc(CN2CCCCC2)cc1</smiles>

(Z)-5-Methyl-8-p-tolyloct-5-enenitrile (33ba): $98 \%$ yield. $\mathrm{SiO}_{2}$ column chromatography (hexane-EtOAc $=20: 1$ ); colorless oil; TLC, $R_{\mathrm{f}}=0.44$ (hexane-EtOAc $\left.=4: 1\right) ;{ }^{1} \mathrm{H}$ NMR $\left(\mathrm{CDCl}_{3}, 300\right.$ MHz) $\delta 1.55-1.66(\mathrm{~m}, 2 \mathrm{H}), 1.66(\mathrm{~s}, 3 \mathrm{H}), 2.09(\mathrm{t}, J=7.5 \mathrm{~Hz}, 2 \mathrm{H}), 2.16(\mathrm{t}, J=7.5 \mathrm{~Hz}, 2 \mathrm{H}), 2.25-2.32(\mathrm{~m}$, $2 \mathrm{H}), 2.32(\mathrm{~s}, 3 \mathrm{H}), 2.60(\mathrm{t}, J=7.5 \mathrm{~Hz}, 2 \mathrm{H}), 5.27(\mathrm{t}, J=7.2 \mathrm{~Hz}, 1 \mathrm{H}), 7.04-7.11(\mathrm{~m}, 4 \mathrm{H}) ;{ }^{13} \mathrm{C} \mathrm{NMR}$ $\left(\mathrm{CDCl}_{3}, 75 \mathrm{MHz}\right) \delta 15.8,20.6,22.5,23.1,29.6,29.9,35.3,119.3,126.1,128.0$ (2C), 128.5 (2C), 132.7, 134.7, 138.4.<smiles>Cc1ccc2c(c1)CC1CCCN2CC1</smiles>

(Z)-5-Methyl-8- $m$-tolyloct-5-enenitrile $\quad(33 b b): \quad 98 \%$ yield. $\quad \mathrm{SiO}_{2} \quad$ column chromatography (hexane-EtOAc $=20: 1$ ); colorless oil; TLC, $R_{\mathrm{f}}=0.54$ (hexane-EtOAc $=4: 1$ ); IR (film) $3013,2957,2934,2860,1715,1604,1452,1363,1165 \mathrm{~cm}^{-1} ;{ }^{1} \mathrm{H}$ NMR $\left(\mathrm{CDCl}_{3}, 300 \mathrm{MHz}\right) \delta 1.56-1.67$ $(\mathrm{m}, 2 \mathrm{H}), 1.66(\mathrm{~d}, J=1.2 \mathrm{~Hz}, 3 \mathrm{H}), 2.10(\mathrm{t}, J=7.5 \mathrm{~Hz}, 2 \mathrm{H}), 2.26-2.33(\mathrm{~m}, 2 \mathrm{H}), 2.33(\mathrm{~s}, 3 \mathrm{H}), 2.60(\mathrm{t}, J=$ $7.5 \mathrm{~Hz}, 2 \mathrm{H}), 5.28(\mathrm{t}, J=7.5 \mathrm{~Hz}, 1 \mathrm{H}), 6.97-7.01(\mathrm{~m}, 3 \mathrm{H}), 7.17-7.20(\mathrm{t}, J=7.8 \mathrm{~Hz}, 1 \mathrm{H}) ;{ }^{13} \mathrm{C} \mathrm{NMR}$ $\left(\mathrm{CDCl}_{3}, 75 \mathrm{MHz}\right) \delta 15.9,21.0,22.6,23.1,29.6,29.9,35.7,119.4,125.2,126.2,127.8,129.0,132.8$, 137.3, 141.5; HRMS (FAB) $\mathrm{m} / z$ calcd for $\mathrm{C}_{16} \mathrm{H}_{22} \mathrm{~N}(\mathrm{M}+\mathrm{H}) 228.1752$ found 228.1759 .<smiles>CCCCCCCCCc1ccc(C)cc1</smiles>

(5E,9E)-5,9-Dimethyl-12-p-tolyldodeca-5,9-dienenitrile (33ca): $97 \%$ yield. $\mathrm{SiO}_{2}$ column chromatography (hexane-EtOAc $\left.=10: 1\right)$; colorless oil; TLC, $R_{\mathrm{f}}=0.55$ (hexane-EtOAc $=$ 4:1); IR (film) 3009, 2924, 2857, 2249, 1515, 1455, 1385, 1107, $815 \mathrm{~cm}^{-1} ;{ }^{1} \mathrm{H} \mathrm{NMR}\left(\mathrm{CDCl}_{3}, 300 \mathrm{MHz}\right)$ $\delta 1.56(\mathrm{~s}, 3 \mathrm{H}), 1.57(\mathrm{~s}, 3 \mathrm{H}), 1.71$ (quintet, $J=7.2 \mathrm{~Hz}, 2 \mathrm{H}), 1.95-2.02(\mathrm{~m}, 2 \mathrm{H}), 2.05-2.11(\mathrm{~m}, 4 \mathrm{H}), 2.22$ $(\mathrm{t}, J=7.2 \mathrm{~Hz}, 2 \mathrm{H}), 2.26-2.30(\mathrm{~m}, 2 \mathrm{H}), 2.30(\mathrm{~s}, 3 \mathrm{H}), 2.59(\mathrm{dd}, J=6.7,9.0 \mathrm{~Hz}, 2 \mathrm{H}), 5.13-5.20(\mathrm{~m}, 2 \mathrm{H})$, 6.98-7.07 (s, 4H); ${ }^{13} \mathrm{C} \mathrm{NMR}\left(\mathrm{CDCl}_{3}, 75 \mathrm{MHz}\right) \delta$ 15.4, 15.8, 16.0, 20.8, 23.2, 26.3, 29.9, 35.5, 38.0, 39.3, 
119.7, 123.8, 126.4, 128.1 (2C), 128.7 (2C), 132.1, 134.8, 135.1, 139.1; HRMS (FAB) $\mathrm{m} / z$ calcd for $\mathrm{C}_{21} \mathrm{H}_{30} \mathrm{~N}(\mathrm{M}+\mathrm{H})$ 296.2378, found 296.2386.<smiles>CCCCCC1=CCC2=CC(c3cccc(C)c3)CC2=C1</smiles>

$(\mathbf{5 E}, 9 E)-5,9-D i m e t h y l-12-m$-tolyldodeca-5,9-dienenitrile (33cb): $93 \%$ yield. $\mathrm{SiO}_{2}$ column chromatography (hexane-EtOAc $=10: 1$ ); colorless oil; TLC, $R_{\mathrm{f}}=0.63$ (hexane-EtOAc $=4: 1$ ); IR (film) 3011, 2925, 2857, 2249, 1607, 1456, 1385, $1094 \mathrm{~cm}^{-1} ;{ }^{1} \mathrm{H} \mathrm{NMR}\left(\mathrm{CDCl}_{3}, 300 \mathrm{MHz}\right) \delta 1.56(\mathrm{~s}$, $3 \mathrm{H}), 1.58(\mathrm{~s}, 3 \mathrm{H}), 1.72$ (quintet, $J=7.2 \mathrm{~Hz}, 2 \mathrm{H}), 2.02(\mathrm{q}, J=7.2 \mathrm{~Hz}, 2 \mathrm{H}), 2.08(\mathrm{q}, J=7.2 \mathrm{~Hz}, 4 \mathrm{H})$, 2.22-2.32 (m, 4H), $2.32(\mathrm{~s}, 3 \mathrm{H}), 2.59(\mathrm{t}, J=6.6 \mathrm{~Hz}, 2 \mathrm{H}), 5.17(\mathrm{dt}, J=6.9 \mathrm{~Hz}, 2 \mathrm{H}), 6.98-7.00(\mathrm{~m}, 3 \mathrm{H})$, $7.18(\mathrm{q}, J=7.5 \mathrm{~Hz}, 1 \mathrm{H}) ;{ }^{13} \mathrm{C} \mathrm{NMR}\left(\mathrm{CDCl}_{3}, 75 \mathrm{MHz}\right) \delta 15.5,15.8,16.0,21.3,23.2,26.4,30.0,35.9$, 38.1, 39.4, 119.7, 123.9, 125.3, 126.3, 126.4, 128.0, 129.2, 132.2, 135.2, 137.6, 142.2; HRMS (FAB) $\mathrm{m} / \mathrm{z}$ calcd for $\mathrm{C}_{21} \mathrm{H}_{30} \mathrm{~N}(\mathrm{M}+1) 296.2378$, found 296.2378.

General Procedure for the Transformation from Nitriles 33 to Ketones 34 (Step j, Scheme 4): To a solution of 33 (5.54 mmol) in dry $\mathrm{Et}_{2} \mathrm{O}$ was added $\mathrm{MeLi}\left(1.0 \mathrm{M}\right.$ in $\mathrm{Et}_{2} \mathrm{O}$, Kanto, $16.6 \mathrm{~mL}, 16.6$ mmol) dropwise at $-78{ }^{\circ} \mathrm{C}$ and then the resulting mixture was allowed to warm to $0{ }^{\circ} \mathrm{C}$. The resulting mixture was stirred for $1 \mathrm{~h}$ and then saturated $\mathrm{NH}_{4} \mathrm{Cl}(50 \mathrm{~mL})$ was added while stirring for another 10 min. at $0{ }^{\circ} \mathrm{C}$. The layers were separated and the organic layer was washed with saturated $\mathrm{NaHCO}_{3}$, the aqueous layer was extracted with $\mathrm{Et}_{2} \mathrm{O}$ (twice) the combined organic layers was dried over anhydrous $\mathrm{MgSO}_{4}$ and concentrated in vacuo.<smiles>O=C1CCCC(CCCc2ccc(F)cc2)C1</smiles>

(E)-9-(4-Fluorophenyl)-6-methylnon-6-en-2-one (34aa): $96 \%$ yield for 2 steps. $\mathrm{SiO}_{2}$ column chromatography (hexane-EtOAc $=20: 1$ ); colorless oil; TLC, $R_{\mathrm{f}}=0.45$ (hexane-EtOAc $=4: 1$ ); IR (film) 3011, 2937, 2859, 1710 (C=O), 1603, 1509, 1362, 1223, 1157, 830; ${ }^{1} \mathrm{H}$ NMR $\left(\mathrm{CDCl}_{3}, 300\right.$ $\mathrm{MHz}) \delta 1.52(\mathrm{~s}, 3 \mathrm{H}), 1.65$ (quintet, $J=7.5 \mathrm{~Hz}, 2 \mathrm{H}), 1.96(\mathrm{t}, J=7.2 \mathrm{~Hz}, 2 \mathrm{H}), 2.12(\mathrm{~s}, 3 \mathrm{H}), 2.27(\mathrm{t}, J=$ $7.5 \mathrm{~Hz}, 2 \mathrm{H}), 2.33(\mathrm{t}, J=7.5 \mathrm{~Hz}, 2 \mathrm{H}), 2.61(\mathrm{t}, J=7.8 \mathrm{~Hz}, 2 \mathrm{H}), 5.13(\mathrm{dt}, J=0.9,7.2 \mathrm{~Hz}, 1 \mathrm{H}), 6.91-6.99$ $(\mathrm{m}, 2 \mathrm{H}), 7.09-7.16(\mathrm{~m}, 2 \mathrm{H}) ;{ }^{13} \mathrm{C} \mathrm{NMR}\left(\mathrm{CDCl}_{3}, 75 \mathrm{MHz}\right) \delta 15.4,21.5,29.6(2 \mathrm{C}), 35.0,38.6,42.6,114.5$, $114.8,123.9,129.5,129.6,135.0,137.6,160.9\left(\mathrm{~d}, J_{\mathrm{CF}}=242\right), 208.7 ;{ }^{19} \mathrm{~F} \mathrm{NMR}\left(\mathrm{CDCl}_{3}, 282 \mathrm{MHz}\right) \delta-$ $118.5\left(\mathrm{dd}, J_{\mathrm{CF}}=9.0,15 \mathrm{~Hz}\right)$; HRMS (FAB) $\mathrm{m} / z$ calcd for $\mathrm{C}_{16} \mathrm{H}_{22} \mathrm{OF}(\mathrm{M}+\mathrm{H}) 249.1655$, found 249.1655.<smiles>CC1=CCCc2ccccc21</smiles>

(E)-6-Methyl-9-phenylnon-6-en-2-one (34ab): $96 \%$ yield for 2 steps. $\mathrm{SiO}_{2}$ column chromatography (hexane-EtOAc $=20: 1$ ); colorless oil; TLC, $R_{\mathrm{f}}=0.45$ (hexane-EtOAc $\left.=4: 1\right)$; IR $($ film) 3013, 2937, 2858, 1710 (C=O), 1603, 1496, 1453, 1362, 1159, $700 \mathrm{~cm}^{-1} ;{ }^{1} \mathrm{H} \mathrm{NMR}\left(\mathrm{CDCl}_{3}, 300 \mathrm{MHz}\right) \delta$ $1.53(\mathrm{~s}, 3 \mathrm{H}), 1.65$ (quintet, $J=7.5 \mathrm{~Hz}, 2 \mathrm{H}), 1.96(\mathrm{t}, J=7.5 \mathrm{~Hz}, 2 \mathrm{H}), 2.11(\mathrm{~s}, 3 \mathrm{H}), 2.31(\mathrm{q}, J=7.5 \mathrm{~Hz}$, $2 \mathrm{H}), 2.33(\mathrm{t}, J=7.5 \mathrm{~Hz}, 2 \mathrm{H}), 2.64(\mathrm{t}, J=7.8 \mathrm{~Hz}, 2 \mathrm{H}), 5.16(\mathrm{dt}, J=1.2,7.2 \mathrm{~Hz}, 1 \mathrm{H}), 7.15-7.20(\mathrm{~m}, 3 \mathrm{H})$. 7.25-7,30 (m, 2H); ${ }^{13} \mathrm{C} \mathrm{NMR}\left(\mathrm{CDCl}_{3}, 75 \mathrm{MHz}\right) \delta 15.3,21.3,29.5$ (2C), 35.7, 38.5, 42.4, 124.1, 125.4, 127.9 (2C), 128.1 (2C), 134.6, 141.8, 208.4; HRMS (FAB) $\mathrm{m} / z$ calcd for $\mathrm{C}_{16} \mathrm{H}_{23} \mathrm{O}(\mathrm{M}+\mathrm{H})$ 231.1749, 


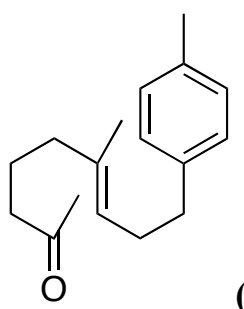

(E)-6-Methyl-9-p-tolylnon-6-en-2-one (34ac): 94\% yield. $\mathrm{SiO}_{2}$ column chromatography (hexane-EtOAc $=20: 1$ ); colorless oil; TLC, $R_{\mathrm{f}}=0.51$ (hexane-EtOAc $=4: 1$ ); IR (film) 3011, 2925, 2864, $1710(\mathrm{C}=\mathrm{O}), 1515,1455,1362,1159,810 \mathrm{~cm}^{-1} ;{ }^{1} \mathrm{H} \mathrm{NMR}\left(\mathrm{CDCl}_{3}, 300 \mathrm{MHz}\right) \delta 1.54(\mathrm{~s}, 3 \mathrm{H}), 1.65$ (quintet, $J=7.5 \mathrm{~Hz}, 2 \mathrm{H}), 1.96(\mathrm{t}, J=7.5 \mathrm{~Hz}, 2 \mathrm{H}), 2.11(\mathrm{~S}, 3 \mathrm{H}), 2.25-2.36(\mathrm{~m}, 4 \mathrm{H}), 2.31(\mathrm{~s}, 3 \mathrm{H}), 2.60(\mathrm{t}$, $J=7.8 \mathrm{~Hz}, 2 \mathrm{H}), 5.16(\mathrm{tq}, J=1.2,7.2 \mathrm{~Hz}, 1 \mathrm{H}), 7.08(\mathrm{~s}, 4 \mathrm{H}) ;{ }^{13} \mathrm{C}$ NMR $\left(\mathrm{CDCl}_{3}, 75 \mathrm{MHz}\right) \delta 15.3,17.5$, 20.7, 21.3, 29.5, 29.6, 35.3, 38.5, 42.4, 124.3, 128.0 (2C), 128.6 (2C), 134.4, 134.6, 138.7, 208.5; HRMS (EI) $m / z$ calcd for $\mathrm{C}_{17} \mathrm{H}_{24} \mathrm{O}(\mathrm{M}+\mathrm{H}) 244.1827$, found 244.1837.<smiles>CCCCCCC(=O)CCCc1cccc(C)c1</smiles>

(E)-6-Methyl-9-m-tolylnon-6-en-2-one (1): 97\% yield. $\mathrm{SiO}_{2}$ column chromatography (hexane-EtOAc = 20:1); colorless oil; TLC, $R_{\mathrm{f}}=0.48$ (hexane-EtOAc $=4: 1$ ); IR (film) 3019, 2929, 2868, $1709(\mathrm{C}=\mathrm{O}), 1457,1362,1158 \mathrm{~cm}^{-1}$; ${ }_{1}^{1} \mathrm{H} \mathrm{NMR}\left(\mathrm{CDCl}_{3}, 300 \mathrm{MHz}\right) \delta 1.50$ (s, 3H), 1.60 (quintet, $J$ $=7.2 \mathrm{~Hz}, 2 \mathrm{H}), 1.91(\mathrm{t}, J=7.2 \mathrm{~Hz}, 2 \mathrm{H}), 1.98(\mathrm{~s}, 3 \mathrm{H}), 2.20-2.30(\mathrm{~m}, 4 \mathrm{H}), 2.27(\mathrm{~s}, 3 \mathrm{H}), 2.56(\mathrm{t}, J=7.8 \mathrm{~Hz}$, $2 \mathrm{H}), 5.13(\mathrm{t}, J=7.2 \mathrm{~Hz}, 1 \mathrm{H}), 6.91-6.94(\mathrm{~m}, 3 \mathrm{H}), 7.09(\mathrm{t}, J=7.5 \mathrm{~Hz}, 1 \mathrm{H}) ;{ }^{13} \mathrm{C} \mathrm{NMR}\left(\mathrm{CDCl}_{3}, 75 \mathrm{MHz}\right) \delta$ 15.0, 20.8, 21.1, 29.1, 29.3, 35.4, 38.3, 42.0, 123.9, 124.9, 125.9, 127.5, 128.7, 134.2, 136.9, 141.4, 207.6; HRMS (EI) $m / z$ calcd for $\mathrm{C}_{17} \mathrm{H}_{24} \mathrm{O}(\mathrm{M}+\mathrm{H})$ 245.1905, found 244.1819.<smiles>CCCCC(=O)CCCc1cccc(CCC)c1</smiles>

(E)-9-(3-Isopropylphenyl)-6-methylnon-6-en-2-one (34ae): $96 \%$ yield. $\quad \mathrm{SiO}_{2}$ column chromatography (hexane-EtOAc $=20: 1$ ); colorless oil; TLC, $R_{\mathrm{f}}=0.46$ (hexane-EtOAc $=4: 1$ ); ${ }^{1} \mathrm{H} \mathrm{NMR}\left(\mathrm{CDCl}_{3}, 300 \mathrm{MHz}\right) \delta 1.24(\mathrm{~d}, J=6.9 \mathrm{~Hz}, 6 \mathrm{H}), 1.54(\mathrm{~s}, 3 \mathrm{H}), 1.66$ (quintet, $\left.J=7.5 \mathrm{~Hz}, 2 \mathrm{H}\right), 1.97$ $(\mathrm{t}, J=7.2 \mathrm{~Hz}, 2 \mathrm{H}), 2.11(\mathrm{~s}, 3 \mathrm{H}), 2.27-2.37(\mathrm{~m}, 4 \mathrm{H}), 2.63(\mathrm{t}, J=7.8 \mathrm{~Hz}, 2 \mathrm{H}), 2.80-2.94(\mathrm{~m}, 1 \mathrm{H}), 5.17$ $(\mathrm{tq}, J=1.2,6.3 \mathrm{~Hz}, 1 \mathrm{H}), 6.99-7.06(\mathrm{~m}, 3 \mathrm{H}), 7.18-7.23(\mathrm{~m}, 1 \mathrm{H}) ;{ }^{13} \mathrm{C}$ NMR $\left(\mathrm{CDCl}_{3}, 75 \mathrm{MHz}\right) \delta 15.5$, 21.6, 23.9 (2C), 29.7, 29.8, 33.9, 35.9, 38.7, 42.7, 123.6, 124.4, 125.7, 126.4, 128.0, 134.6, 142.0, 148.6, $208.8(\mathrm{C}=\mathrm{O})$.<smiles>Cc1ccc(CC(=O)C2CC3CCC2C3)cc1</smiles>

(Z)-6-Methyl-9-p-tolylnon-6-en-2-one (34ba): 97\% yield. $\mathrm{SiO}_{2}$ column chromatography (hexane-EtOAc = 10:1); colorless oil; TLC, $R_{\mathrm{f}}=0.45$ (hexane-EtOAc $=4: 1$ ); IR (film) 3010, 2929, 2857, 2359, $1710(\mathrm{C}=\mathrm{O}), 1515,1456,1362,1162,810 \mathrm{~cm}^{-1} ;{ }^{1} \mathrm{H}$ NMR $\left(\mathrm{CDCl}_{3}, 300 \mathrm{MHz}\right) \delta 1.55-1.67$ $(\mathrm{m}, 2 \mathrm{H}), 1.66(\mathrm{~d}, J=1.2 \mathrm{~Hz}, 3 \mathrm{H}), 1.99(\mathrm{t}, J=7.8 \mathrm{~Hz}, 2 \mathrm{H}), 2.11(\mathrm{~s}, 3 \mathrm{H}), 2.22-2.36(\mathrm{~m}, 2 \mathrm{H}), 2.31(\mathrm{~s}, 3 \mathrm{H})$, $2.59(\mathrm{t}, J=7.2 \mathrm{~Hz}, 2 \mathrm{H}), 5.21(\mathrm{t}, J=7.2 \mathrm{~Hz}, 1 \mathrm{H}), 7.08(\mathrm{~s}, 4 \mathrm{H}) ;{ }^{13} \mathrm{C} \mathrm{NMR}\left(\mathrm{CDCl}_{3}, 75 \mathrm{MHz}\right) \delta 20.8,21.5$, 
22.9, 29.6, 29.8, 30.6, 35.6, 42.8, 125.0, 128.1 (2C), 128.7 (2C), 134.6, 134.8, 138.8, $208.5(\mathrm{C}=\mathrm{O})$; HRMS (FAB) $m / z$ calcd for $\mathrm{C}_{17} \mathrm{H}_{25} \mathrm{O}(\mathrm{M}+\mathrm{H}) 245.1905$, found 245.1898.

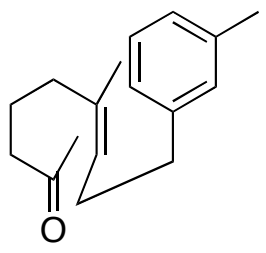

(Z)-6-Methyl-9-m-tolylnon-6-en-2-one (34bb): $95 \%$ yield. $\quad \mathrm{SiO}_{2}$ column chromatography (hexane-EtOAc $=20: 1)$; colorless oil; TLC, $R_{\mathrm{f}}=0.54$ (hexane-EtOAc $\left.=4: 1\right)$; IR $($ film) 3019, 2931, 2858, 1710 (C=O), 1456, 1362, 1213, 1161, 1044, $928 \mathrm{~cm}^{-1} ;{ }_{1}^{1} \mathrm{H} \mathrm{NMR}\left(\mathrm{CDCl}_{3}, 300 \mathrm{MHz}\right) \delta$ 1.56-1.67 (m, 2H), $1.67(\mathrm{~s}, 3 \mathrm{H}), 1.99(\mathrm{t}, J=7.6 \mathrm{~Hz}, 2 \mathrm{H}), 2.12(\mathrm{~s}, 3 \mathrm{H}), 2.23-2.37(\mathrm{~m}, 2 \mathrm{H}), 2.33(\mathrm{~s}, 3 \mathrm{H})$, $2.59(\mathrm{t}, J=7.8 \mathrm{~Hz}, 2 \mathrm{H}), 5.21(\mathrm{t}, J=7.2 \mathrm{~Hz}, 1 \mathrm{H}), 6.98-7.00(\mathrm{~m}, 3 \mathrm{H}), 7.17-7.20(\mathrm{~m}, 1 \mathrm{H}) ;{ }^{13} \mathrm{C} \mathrm{NMR}$ $\left(\mathrm{CDCl}_{3}, 75 \mathrm{MHz}\right) \delta 21.0,21.4,22.8,29.4,29.6,30.5,35.9,42.6,124.9,125.1,126.1,127.8,128.9$, 134.5, 137.2, 141.7, 208.0; HRMS (FAB) $m / z$ calcd for $\mathrm{C}_{17} \mathrm{H}_{25} \mathrm{O}(\mathrm{M}+\mathrm{H})$ 245.1905, found 245.1904.<smiles>CC1=C2C=C(CCCc3ccc(C)cc3)C=C2CCC1</smiles>

(6E,10E)-6,10-Dimethyl-13-p-tolyltrideca-6,10-dien-2-one (34ca): $95 \%$ yield. $\mathrm{SiO}_{2}$ column chromatography (hexane-EtOAc $=10: 1$ ); colorless oil; TLC $R_{\mathrm{f}}=0.51$ (hexane-EtOAc $=$ 4:1); IR (film) 3010, 2925, 2857, $1710(\mathrm{C}=\mathrm{O}), 1515,1456,1362,1227,1158 \mathrm{~cm}^{-1}$; ${ }^{1} \mathrm{H} \mathrm{NMR}\left(\mathrm{CDCl}_{3}\right.$, $300 \mathrm{MHz}) \delta 1.56(\mathrm{~s}, 3 \mathrm{H}), 1.57(\mathrm{~s}, 3 \mathrm{H}), 1.67$ (quintet, $J=7.5 \mathrm{~Hz}, 2 \mathrm{H}), 1.93-2.00(\mathrm{~m}, 4 \mathrm{H}), 2.04-2.11(\mathrm{~m}$, $2 \mathrm{H}), 2.11(\mathrm{~s}, 3 \mathrm{H}), 2.24-2.31(\mathrm{~m}, 2 \mathrm{H}), 2.36(\mathrm{t}, J=7.5 \mathrm{~Hz}, 2 \mathrm{H}), 2.31(\mathrm{~s}, 3 \mathrm{H}), 2.59(\mathrm{dd}, J=7.9,9.9 \mathrm{~Hz}$, $2 \mathrm{H}), 5.10(\mathrm{dt}, J=1.2,6.9 \mathrm{~Hz}, 1 \mathrm{H}), 5.18(\mathrm{dt}, J=1.2,7.2 \mathrm{~Hz}, 1 \mathrm{H}), 7.08(\mathrm{~s}, 4 \mathrm{H}) ;{ }^{13} \mathrm{C} \mathrm{NMR}\left(\mathrm{CDCl}_{3}, 75\right.$ $\mathrm{MHz}) \delta 15.6,15.9,20.9,21.7,26.4,29.9,30.0,35.6,38.8,39.6,42.8,123.7,125.0,128.2$ (2C), 128.8 (2C), 134.0, 134.9, 135.4, 139.2, 209.1; HRMS (FAB) $\mathrm{m} / z$ calcd for $\mathrm{C}_{22} \mathrm{H}_{32} \mathrm{ONa}(\mathrm{M}+\mathrm{Na}) 335.2351$, found 335.2348 .

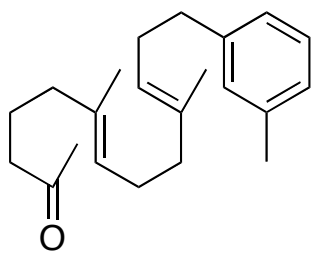

$(6 E, 10 E)-6,10-D i m e t h y l-13-p-t o l y l t r i d e c a-6,10-d i e n-2-o n e \quad(34 c b): 96 \%$ yield. $\mathrm{SiO}_{2}$ column chromatography (hexane-EtOAc $=10: 1$ ); colorless oil; $\mathrm{TLC}, R_{\mathrm{f}}=0.48$ (hexane-EtOAc $=$ 4:1); IR (film) 3010, 2925, 2857, 1710 (C=O), 1607, 1450, 1362, $1158 \mathrm{~cm}^{-1} ;{ }^{1} \mathrm{H} \mathrm{NMR}\left(\mathrm{CDCl}_{3}, 300\right.$ $\mathrm{MHz}) \delta 1.57(\mathrm{~s}, 3 \mathrm{H}), 1.58(\mathrm{~s}, 3 \mathrm{H}), 1.67$ (quintet, $J=7.2 \mathrm{~Hz}, 2 \mathrm{H}), 1.94-2.09(\mathrm{~m}, 6 \mathrm{H}), 2.13(\mathrm{~s}, 3 \mathrm{H}), 2.25-$ $2.33(\mathrm{~m}, 4 \mathrm{H}), 2.33(\mathrm{~s}, 3 \mathrm{H}), 2.59(\mathrm{dd}, J=7.5,9.9 \mathrm{~Hz}, 2 \mathrm{H}), 5.10(\mathrm{dt}, J=1.2,6.9 \mathrm{~Hz}, 1 \mathrm{H}), 5.19(\mathrm{dt}, J=1.2$, $7.2 \mathrm{~Hz}, 1 \mathrm{H}), 6.99-7.01(\mathrm{~m}, 3 \mathrm{H}), 7.17(\mathrm{dt}, J=1.5,7.5 \mathrm{~Hz}, 1 \mathrm{H}) ;{ }^{13} \mathrm{C} \mathrm{NMR}\left(\mathrm{CDCl}_{3}, 75 \mathrm{MHz}\right) \delta 15.5,15.8$, 21.3, 21.6, 26.4, 29.8, 29.9, 35.9, 38.7, 39.5, 42.7, 123.6, 124.9, 125.3, 126.3, 128.0, 129.1, 133.9, 135.4, 137.5, 142.1, 208.9; HRMS (FAB) $m / z$ calcd for $\mathrm{C}_{22} \mathrm{H}_{33} \mathrm{O}(\mathrm{M}+\mathrm{H}) 313.2531$, found 313.2526.

General Procedure for the Transformation from Nitriles 33 to Aldehydes 13 (Step k, Scheme 3): To a solution of $\mathbf{3 3}(782 \mathrm{mg}, 3.44 \mathrm{mmol})$ in toluene-hexane (1:2 v/v, $45 \mathrm{~mL})$ was added DIBAL-H (1.0 $\mathrm{M}$ in toluene, Kanto, $30 \mathrm{~mL}, 30.0 \mathrm{mmol}$ ) at $-78^{\circ} \mathrm{C}$ slowly with stirring and the resulting mixture was stirred for $1 \mathrm{~h}$ at $-78{ }^{\circ} \mathrm{C}$. Acetone $(5 \mathrm{~mL})$, EtOAc $(5 \mathrm{~mL})$, buffer $(p \mathrm{H} 7,3 \mathrm{~mL})$ was added and stirred for $20 \mathrm{~min}\left(-78{ }^{\circ} \mathrm{C}\right.$ to $\left.0{ }^{\circ} \mathrm{C}\right)$. Then $\mathrm{Na}_{2} \mathrm{SO}_{4}$ was added and stirred for additional 30 min at room temperature. The resulting mixture was filtered through celite and $\mathrm{Na}_{2} \mathrm{SO}_{4}$ and solvents were removed 
under vacuo to give crude products $\mathbf{1 3}$.<smiles>CCCCCCCC(=O)CCCc1ccc(C)cc1</smiles>

(E)-5-Methyl-8-p-tolyloct-5-enal (13a): $94 \%$ yield. $\mathrm{SiO}_{2}$ column chromatography (hexane-EtOAc $=10: 1)$; colorless oil; TLC, $R_{\mathrm{f}}=0.48$ (hexane-EtOAc $\left.=4: 1\right) ;{ }^{1} \mathrm{H} \mathrm{NMR}\left(\mathrm{CDCl}_{3}, 300\right.$ $\mathrm{MHz}) \delta 1.54(\mathrm{~s}, 3 \mathrm{H}), 1.71(\mathrm{qn}, J=7.2 \mathrm{~Hz}, 2 \mathrm{H}), 2.00(\mathrm{t}, J=7.2 \mathrm{~Hz}, 2 \mathrm{H}), 2.25-2.35(\mathrm{~m}, 4 \mathrm{H}), 2.32(\mathrm{~s}, 3 \mathrm{H})$, $2.60(\mathrm{t}, J=7.8 \mathrm{~Hz}, 2 \mathrm{H}), 5.17(\mathrm{tq}, J=1.2,6.9 \mathrm{~Hz}, 1 \mathrm{H}), 7.08(\mathrm{~s}, 4 \mathrm{H}), 9.74(\mathrm{t}, J=1.8 \mathrm{~Hz}, 1 \mathrm{H}) ;{ }^{13} \mathrm{C} \mathrm{NMR}$ $\left(\mathrm{CDCl}_{3}, 75 \mathrm{MHz}\right) \delta$ 15.3, 19.8, 20.7, 29.7, 35.2, 38.5, 42.7, 124.7, 128.0 (2C), 128.6 (2C), 134.1, 134.7, 138.7, 202.0.<smiles>Cc1cccc(CCCC(=O)CCCC(C)C)c1</smiles>

(E)-5-Methyl-8-m-tolyloct-5-enal (13b): $96 \%$ yield. $\mathrm{SiO}_{2}$ column chromatography (hexane-EtOAc $=10: 1)$; colorless oil; TLC, $R_{\mathrm{f}}=0.48$ (hexane-EtOAc $\left.=4: 1\right) ;{ }^{1} \mathrm{H} \mathrm{NMR}\left(\mathrm{CDCl}_{3}, 300\right.$ $\mathrm{MHz}) \delta 1.55(\mathrm{~s}, 3 \mathrm{H}), 1.71$ (quintet, $J=7.2 \mathrm{~Hz}, 2 \mathrm{H}), 2.00(\mathrm{t}, J=7.2 \mathrm{~Hz}, 2 \mathrm{H}), 2.26-2.36(\mathrm{~m}, 4 \mathrm{H}), 2.33(\mathrm{~s}$, $3 \mathrm{H}), 2.61(\mathrm{dd}, J=7.2,8.1 \mathrm{~Hz}, 2 \mathrm{H}), 5.18(\mathrm{ddq}, J=1.2,2.4,7.2 \mathrm{~Hz}, 1 \mathrm{H}), 6.97-7.00(\mathrm{~m}, 3 \mathrm{H}), 7.17(\mathrm{t}, J=$ $7.8 \mathrm{~Hz}, 1 \mathrm{H}) ;{ }^{13} \mathrm{C} \mathrm{NMR}\left(\mathrm{CDCl}_{3}, 75 \mathrm{MHz}\right) \delta 15.4,19.8,21.1,29.6,35.6,38.5,42.7,124.7,125.2,126.2$, $127.9,129.0,134.2,137.3,141.8,202.1$.<smiles>CCCCC(=O)CCCc1cccc(CCC)c1</smiles>

(E)-8-(3-Isopropylphenyl)-5-methyloct-5-enal (13c): $93 \%$ yield. $\mathrm{SiO}_{2}$ column chromatography (hexane-EtOAc $=20: 1)$; colorless oil; TLC, $R_{\mathrm{f}}=0.50$ (hexane-EtOAc $\left.=4: 1\right)$; IR (film) 2962, 2931, 2727, $1721(\mathrm{C}=\mathrm{O}), 1604,1459 \mathrm{~cm}^{-1} ;{ }^{1} \mathrm{H}$ NMR $\left(\mathrm{CDCl}_{3}, 300 \mathrm{MHz}\right) \delta 1.24(\mathrm{~d}, J=6.5 \mathrm{~Hz}$, $6 \mathrm{H}), 1.54(\mathrm{~s}, 3 \mathrm{H}), 1.71$ (quintet, $J=7.2 \mathrm{~Hz}, 2 \mathrm{H}), 2.00(\mathrm{t}, J=7.5 \mathrm{~Hz}, 2 \mathrm{H}), 2.28-2.36(\mathrm{~m}, 4 \mathrm{H}), 2.63(\mathrm{t}, J=$ $7.8 \mathrm{~Hz}, 2 \mathrm{H}), 2.80-2.94(\mathrm{~m}, 1 \mathrm{H}), 5.18(\mathrm{dt}, J=1.2,7.2 \mathrm{~Hz}, 1 \mathrm{H}), 6.99-7.06(\mathrm{~m}, 3 \mathrm{H}), 7.18-7.23(\mathrm{~m}, 1 \mathrm{H})$, $9.73(\mathrm{t}, J=1.2 \mathrm{~Hz}, 1 \mathrm{H}) ;{ }^{13} \mathrm{C} \mathrm{NMR}\left(\mathrm{CDCl}_{3}, 75 \mathrm{MHz}\right) \delta 15.6,20.0,24.0(2 \mathrm{C}), 29.8,34.0,35.9,38.7,43.0$, 123.7, 124.9, 125.8, 126.5, 128.1, 134.3, 142.0, 148.7, $202.6(\mathrm{C}=\mathrm{O})$; HRMS (FAB) $\mathrm{m} / z$ calcd for $\mathrm{C}_{18} \mathrm{H}_{27} \mathrm{O}(\mathrm{M}+\mathrm{H})$ 259.2062, found 259.2058.

General Procedure for the Synthesis of Triisopropylsilyl Enol Ethers 4, 7, and 10 from Ketones 34 (Step l, Scheme 4): To a solution of $34(1.80 \mathrm{mmol})$ and $\mathrm{Et}_{3} \mathrm{~N}(627 \mu \mathrm{L}, 4.50 \mathrm{mmol})$ in dry $\mathrm{CH}_{2} \mathrm{Cl}_{2}(3.6 \mathrm{~mL})$ was added TIPSOTf $(473 \mu \mathrm{L}, 1.82 \mathrm{mmol})$ dropwise at $0{ }^{\circ} \mathrm{C}$, and the resulting mixture was stirred for $3 \mathrm{~h}$ at $0{ }^{\circ} \mathrm{C}$. After the reaction was quenched with saturated $\mathrm{NaHCO}_{3}$ and extracted with $\mathrm{Et}_{2} \mathrm{O}$ (twice), the combined organic layers were dried over anhydrous $\mathrm{MgSO}_{4}$ and concentrated in vacuo. The residue was purified by flash chromatography on aluminium oxide 150 basic (type $\mathrm{T}$, deactivated with water) eluted with hexane to give $\mathbf{4 , 7}$, or $\mathbf{1 0}$. The product regio- and stereo-isomeric ratio ${ }^{16}$ was determined by ${ }^{1} \mathrm{H}$ NMR analysis. 
<smiles>FC(F)=C1CCCC=C1CCCc1ccc(F)cc1</smiles>

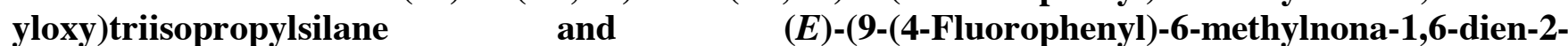
yloxy)triisopropylsilane: $>99 \%$ yield, $(2 E)-2-:(2 Z)-2-: 1-$ enyl-4a $=1: 30: 69$. Colorless oil; TLC, $R_{\mathrm{f}}=$ 0.72 (hexane-EtOAc = 4:1), 0.20 (hexane); IR (film) 2946, 2867, 1671, 1620, 1509, 1464, 1381, 1157 , 1016, $883 \mathrm{~cm}^{-1}$; ${ }^{1} \mathrm{H} \mathrm{NMR}\left(\mathrm{CDCl}_{3}, 300 \mathrm{MHz}\right.$, note: all peaks could not be determined exactly) $\delta 1.06-$ $1.23(\mathrm{~m}, 21 \mathrm{H}), 1.51-1.63(\mathrm{~m}, 2 \mathrm{H}), 151(\mathrm{~s}, 3 \mathrm{H}), 1.76(\mathrm{~s}, 3 \mathrm{H},(2 E)$-2-enyl isomer), $1.82(\mathrm{~s}, 3 \mathrm{H},(2 \mathrm{Z})-2-$ enyl isomer), 1.93-2.02 (m, 4H), 2.09-2.16 (m, 2H, (2E)-2- and (2Z)-2-enyl isomers), $2.28(\mathrm{q}, J=7.5$ $\mathrm{Hz}, 2 \mathrm{H}), 2.32(\mathrm{~s}, 3 \mathrm{H}), 2.60(\mathrm{t}, J=7.5 \mathrm{~Hz}, 2 \mathrm{H}), 3,97$ (s, 1H, 1-enyl isomer), 4.01 (s, 1H, 1-enyl isomer), $4.30(\mathrm{t}, J=6.6 \mathrm{~Hz}, 1 \mathrm{H}, Z$-isomer), $4.61(\mathrm{t}, J=6.6 \mathrm{~Hz}, 1 \mathrm{H},(2 E)$-2-enyl isomer), 5.15 (dt, $J=1.2,7.1 \mathrm{~Hz}$, 1H), 6.90-6.99 (m, 2H), 7.10-7.05 (m, 2H); ${ }^{13} \mathrm{C} \mathrm{NMR}\left(\mathrm{CDCl}_{3}, 75 \mathrm{MHz}\right.$, note: all peaks could not be determined exactly) $\delta 12.6$ (3C, TIPS), 13.2, 15.7, 15.9, 18.0 (6C, TIPS), 22.9, 24.1, 25.2, 30.0, 35.3, 36.2, 39.1, 39.7, 88.8, 107.5, 114.7, 115.0, 123.1, 123.4, 129.7, 129.8, 135.9, 136.2, 137.8, 146.7, 159.6, 162.8; ${ }^{19} \mathrm{~F} \mathrm{NMR}\left(\mathrm{CDCl}_{3}, 282 \mathrm{MHz}\right) \delta-118.7(\mathrm{~m})$; HRMS (FAB) $m / z$ calcd for $\mathrm{C}_{25} \mathrm{H}_{42} \mathrm{OFSi}(\mathrm{M}+\mathrm{H})$ 405.2989, found 405.2987.<smiles>CC1=CCCCC1=CCc1ccccc1</smiles>

A Mixture of Triisopropyl $((2 E, 6 E)$ - and $(2 Z, 6 E)-6-m e t h y l-9-p h e n y l n o n a-2,6-d i e n-2-$ yloxy)silanes and $(E)$-Triisopropyl(6-methyl-9-phenylnona-1,6-dien-2-yloxy)silane (4b): $>99 \%$ yield, $(2 E)-2-:(2 Z)-2-: 1-e n y l-4 b=1: 30: 69$. Colorless oil; TLC, $R_{\mathrm{f}}=0.70$ (hexane-EtOAc $=4: 1$ ), 0.09 (hexane); IR (film) 2946, 2867, 1670, 1622, 1464, 1382, 1268, 1157, 1015, $883 \mathrm{~cm}^{-1} ;{ }^{1} \mathrm{H} \mathrm{NMR}\left(\mathrm{CDCl}_{3}\right.$, $300 \mathrm{MHz}$, note: all peaks could not be determined exactly.) $\delta 1.00-1.26(\mathrm{~m}, 21 \mathrm{H}), 1.53-1.64(\mathrm{~m}, 2 \mathrm{H})$, $153(\mathrm{~s}, 3 \mathrm{H}), 1.76(\mathrm{~s}, 3 \mathrm{H},(2 \mathrm{E})-2$-enyl isomer), 1.82 (s, 3H, (2Z)-2-enyl isomer), 1.96-2.02 (m, 4H), 2.10-2.17 (m, 2H, (2E)-2- and (2Z)-2-enyl isomers), 2.29 (q, $J=7.2 \mathrm{~Hz}, 2 \mathrm{H}), 2.63(\mathrm{t}, J=8.4 \mathrm{~Hz}, 2 \mathrm{H}$ ), $3,97(\mathrm{~s}, 1 \mathrm{H}, 1$-enyl isomer), $4.01(\mathrm{~s}, 1 \mathrm{H}, 1$-enyl isomer), $4.31(\mathrm{t}, J=6.0 \mathrm{~Hz}, 1 \mathrm{H},(2 Z)$-2-enyl isomer), $4.61(\mathrm{t}, J=6.0 \mathrm{~Hz}, 1 \mathrm{H},(2 E)$-2-enyl isomer), $5.18(\mathrm{t}, J=6.9 \mathrm{~Hz}, 1 \mathrm{H}), 7.15-7.20(\mathrm{~m}, 3 \mathrm{H}), 7.25-7.30(\mathrm{~m}$, $2 \mathrm{H}) ;{ }^{13} \mathrm{C} \mathrm{NMR}\left(\mathrm{CDCl}_{3}, 75 \mathrm{MHz}\right.$, note: all peaks could not be determined exactly.) $\delta 21.7$ (6C, TIPS, (2E)-2- and (2Z)-2-enyl isomers), 13.2, 15.7, 15.9, 18.1 (6C, TIPS, 1-enyl isomer), 22.9, 24.1, 25.2, 30.0, 31.7, 36.2, 39.1, 39.7, 88.8, 107.5, 123.4, 123.8, 125.6, 128.2, 128.4, 135.6, 142.3, 146.6, 159.6; HRMS (FAB) $m / z$ calcd for $\mathrm{C}_{25} \mathrm{H}_{43} \mathrm{OSi}(\mathrm{M}+\mathrm{H}) 387.3083$, found 387.3076 .<smiles>Cc1ccc(CCC=C2CCCCC2OC(F)(F)F)cc1</smiles>

A Mixture (4c) of Triisopropyl $((2 E, 6 E)$ - and (2Z,6E)-6-methyl-9-p-tolylnona-2,6dien-2-yloxy)silanes and $(E)$-Triisopropyl(6-methyl-9-p-tolylnona-1,6-dien-2-yloxy)silane: $>99 \%$ yield, $(2 E)-2-:(2 Z)-2-: 1-e n y l-4 c=2: 29: 69$. If a $(2 E)-2-:(2 Z)-2-: 1-e n y l$ ratio of $4 \mathbf{c}$ was changed to 14:71:15 by partial hydrolysis (ca. $50 \%$ conversion) passing through silica column chromatography eluting with hexane. Colorless oil. TLC, $R_{\mathrm{f}}=0.80$ (hexane-EtOAc $=4: 1$ ), 0.22 (hexane); IR (film) 3007, 2946, 2867, 1671, 1514, 1464, 1381, 1250, $1160 \mathrm{~cm}^{-1} ;{ }^{1} \mathrm{H}$ NMR $\left(\mathrm{CDCl}_{3}, 300 \mathrm{MHz}\right.$, note: all peaks could not be determined exactly.) $\delta 1.04-1.26(\mathrm{~m}, 21 \mathrm{H}), 1.55-1.64(\mathrm{~m}, 3 \mathrm{H}), 1.55(\mathrm{~s}, 3 \mathrm{H}), 1.77(\mathrm{~s}$,

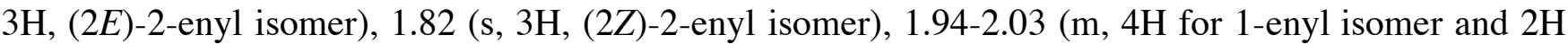


for (2E)- and (2Z)-2-enyl isomers), 2.09-2.18 (m, 2H, (2E)- and (2Z)-2-enyl isomers), 2.22-2.32 (m, 2H), $2.32(\mathrm{~s}, 3 \mathrm{H}), 2.59(\mathrm{t}, J=7.8 \mathrm{~Hz}, 2 \mathrm{H}), 3,98$ (s, 1H, 1-enyl isomer), 4.01 (s, 1H, 1-enyl isomer), 4.31 (dt, $J=1.2,6.9 \mathrm{~Hz}, 1 \mathrm{H},(2 Z)$-2-enyl isomer), 4.62 (t, $J=7.8 \mathrm{~Hz}, 1 \mathrm{H},(2 E)$-2-enyl isomer), 5.19 (dt, $J=$ 1.2, $7.2 \mathrm{~Hz}, 1 \mathrm{H}), 7.08(\mathrm{~s}, 4 \mathrm{H}) ;{ }^{13} \mathrm{C} \mathrm{NMR}\left(\mathrm{CDCl}_{3}, 75 \mathrm{MHz}\right.$, note: all peaks could not be determined exactly.) $\delta 12.6$ (3C, TIPS), 13.2, 13.4, 15.8, 15.9, 17.7, 18.1 (6C, TIPS), 18.6, 21.0, 22.9, 24.0, 25.2, 25.7, 30.1, 30.2, 35.7, 36.2, 39.1, 39.7, 40.3, 88.8, 106.5, 107.6, 123.6, 123.9, 124.0, 128.3 (2C), 128.9, $130.6,134.9,135.0,135.4,135.5,135.8,139.3$ (2C), 146.6, 147.9, 159.6; HRMS (FAB) $\mathrm{m} / z$ calcd for $\mathrm{C}_{26} \mathrm{H}_{45} \mathrm{OSi}(\mathrm{M}+\mathrm{H})$ 401.3240, found 401.3225.

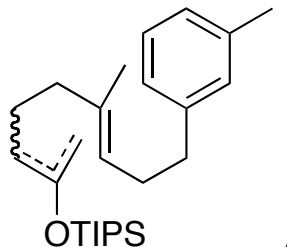

A Mixture (4d) of Triisopropyl((2E,6E)- and $(2 Z, 6 E)-6$-methyl-9-m-tolylnona-2,6dien-2-yloxy)silanes and $(E)$-Triisopropyl(6-methyl-9-m-tolylnona-1,6-dien-2-yloxy)silane: $>99 \%$ yield, (2E)-2-:(2Z)-2-:1-enyl-4d = 2:30:68. Colorless oil; TLC, $R_{\mathrm{f}}=0.80$ (hexane-EtOAc $=4: 1$ ), $R_{\mathrm{f}}=$ 0.22 (hexane); ${ }^{1} \mathrm{H}$ NMR $\left(\mathrm{CDCl}_{3}, 300 \mathrm{MHz}\right.$, note: all peaks could not be determined exactly) $\delta 1.06-1.24$ $(\mathrm{m}, 21 \mathrm{H}), 1.56-1.64(\mathrm{~m}, 1 \mathrm{H}), 1.56(\mathrm{~s}, 3 \mathrm{H}), 1.77$ (s, 3H, (2E)-2-enyl isomer), 1.82 (s, 3H, (2Z)-2-enyl isomer), 1.92-2.04 (m, $4 \mathrm{H}$ for 1-enyl isomer and $2 \mathrm{H}$ for $(2 E)-2$ - and (2Z)-2-enyl isomers), 2.08-2.18 (m, 2H, (2E)-2- and (2Z)-2-enyl isomers), 2.24-2.33 (m, 2H), $2.33(\mathrm{~s}, 3 \mathrm{H}), 2.59(\mathrm{t}, J=7.8 \mathrm{~Hz}, 2 \mathrm{H}), 3,98(\mathrm{~s}$, $1 \mathrm{H}, 1$-enyl isomer), $4.01(\mathrm{~s}, 1 \mathrm{H}, 1$-enyl isomer), $4.32(\mathrm{t}, J=6.9 \mathrm{~Hz}, 1 \mathrm{H},(2 Z)$-2-enyl isomer), $4.63(\mathrm{t}, J=$ $7.2 \mathrm{~Hz}, 1 \mathrm{H},(2 E)-2$-enyl isomer), $5.19(\mathrm{dt}, J=0.9,7.2 \mathrm{~Hz}, 1 \mathrm{H}), 6.98-7.01(\mathrm{~s}, 3 \mathrm{H}), 7.17(\mathrm{t}, J=7.5 \mathrm{~Hz}$, $1 \mathrm{H})$.<smiles>Cc1ccc2c(c1)CCC=C1CCC=C(OC(F)(F)F)C=C1CC2</smiles>

A Mixture (7a) of $((2 E, 6 E, 10 E)-$ and $(2 Z, 6 E, 10 E)-6,10-D i m e t h y l-13-p$ tolyltrideca-2,6,10-trien-2-yloxy)triisopropylsilanes and ((6E,10E)-6,10-Dimethyl-13-ptolyltrideca-1,6,10-trien-2-yloxy)triisopropylsilane: $>99 \%$ yield, $(2 E)-2-:(2 Z)-2-: 1$-enyl-7a = 2:28:70. Colorless oil; TLC, $R_{\mathrm{f}}=0.77$ (hexane-EtOAc $=4: 1$ ); IR (film) 2945, 2867, 1669, 1621, 1464, 1382, 1268, 1157, 1016, $883 \mathrm{~cm}^{-1} ;{ }^{1} \mathrm{H}$ NMR $\left(\mathrm{CDCl}_{3}, 300 \mathrm{MHz}\right.$, note: all peaks could not be determined exactly.) $\delta$ 1.04-1.22 (m, 21H), 1.56-1.64 (m, 2H), 1.57 (s, 3H), 1.60 (s, 3H), 1.76 (s, 3H, (2E)-2-enyl isomer), 1.81 (s, 3H, (2Z)-2-enyl isomer), 1.96-2.18 m, 8H), 2.24-2.32 (m, 2H), 2.32 (s, 3H), 2.59 (dd, $J=6.9,8.7 \mathrm{~Hz}, 2 \mathrm{H}), 3.98$ (s, 1H, 1-enyl isomer), 4.01 (s, 1H, 1-enyl isomer), $4.32(\mathrm{t}, J=6.6 \mathrm{~Hz}, 1 \mathrm{H}$, (2Z)-2-enyl isomer), $4.62(\mathrm{t}, J=7.8 \mathrm{~Hz}, 1 \mathrm{H},(2 E)$-2-enyl isomer), $5.10(\mathrm{t}, J=6.6 \mathrm{~Hz}, 1 \mathrm{H}), 5.19(\mathrm{t}, J=$ $7.2 \mathrm{~Hz}, 1 \mathrm{H}), 7.09(\mathrm{~s}, 4 \mathrm{H}) ;{ }^{13} \mathrm{C} \mathrm{NMR}\left(\mathrm{CDCl}_{3}, 75 \mathrm{MHz}\right.$, note: all peaks could not be determined exactly.)

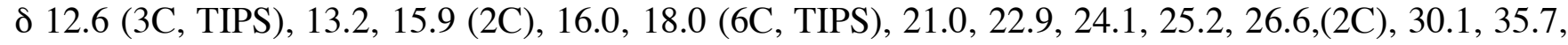
36.1, 39.1, 39.7, 88.8, 107.5, 123.7(2C), 124.1, 124.5, 128.3, 128.9, 134.7, 134.9, 135.1, 135.5, 135.6, 139.3, 146.6, 159.7; HRMS (FAB) $\mathrm{m} / z$ calcd for $\mathrm{C}_{31} \mathrm{H}_{53} \mathrm{OSi}(\mathrm{M}+\mathrm{H}) 469.3866$, found 469.3869 .

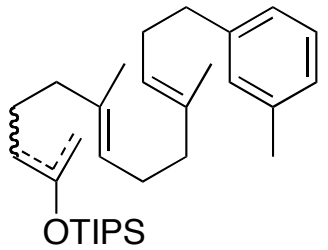

A Mixture (7b) of $((2 E, 6 E, 10 E)-$ and $(2 Z, 6 E, 10 E)-6,10-D i m e t h y l-13-m$ tolyltrideca-2,6,10-trien-2-yloxy)triisopropylsilanes and ((6E,10E)-6,10-Dimethyl-13-mtolyltrideca-1,6,10-trien-2-yloxy)triisopropylsilane: $>99 \%$ yield, $(2 E)-2-:(2 Z)-2-: 1$-enyl-7b = 2:28:70. Colorless oil; TLC, $R_{\mathrm{f}}=0.77$ (hexane-EtOAc $=4: 1$ ); IR (film) 2945, 2867, 1669, 1609, 1464, 1382, 1268, 1157, 1015, 883 $\mathrm{cm}^{-1}$; ${ }^{1} \mathrm{H}$ NMR $\left(\mathrm{CDCl}_{3}, 300 \mathrm{MHz}\right.$, note: all peaks could not be determined exactly.) $\delta 1.06-1.20(\mathrm{~m}, 21 \mathrm{H}), 1.55-1.62(\mathrm{~m}, 2 \mathrm{H}), 1.56(\mathrm{~s}, 3 \mathrm{H}), 1.59(\mathrm{~s}, 3 \mathrm{H}), 1.76(\mathrm{~s}, 3 \mathrm{H},(2 E)-2$-enyl 
isomer), $1.81(\mathrm{~s}, 3 \mathrm{H},(2 \mathrm{Z})-2$-enyl isomer), 1.96-2.16 m, 8H), 2.24-2.31 (m, 2H), $2.31(\mathrm{~s}, 3 \mathrm{H}), 2.59(\mathrm{t}, J$ $=7.8 \mathrm{~Hz}, 2 \mathrm{H}), 3,98(\mathrm{~s}, 1 \mathrm{H}, 1$-enyl isomer $), 4.01(\mathrm{~s}, 1 \mathrm{H}, 1$-enyl isomer $), 4.32(\mathrm{t}, J=6.6 \mathrm{~Hz}, 1 \mathrm{H},(2 Z)-2-$ enyl isomer), $4.61(\mathrm{t}, J=6.6 \mathrm{~Hz}, 1 \mathrm{H},(2 E)$-2-enyl isomer), 5.10-5.21 (m, 2H), 6.96-7.00 (m, 3H), 7.12$7.17(\mathrm{~m}, 1 \mathrm{H}) ;{ }^{13} \mathrm{C} \mathrm{NMR}\left(\mathrm{CDCl}_{3}, 75 \mathrm{MHz}\right.$, note: all peaks could not be determined exactly.) $\delta 12.6$ (3C, TIPS), 13.2, 15.8, 15.9, 18.0 (6C, TIPS), 21.4, 22.9, 24.1, 25.2, 26.6, 26.7, 30.1, 36.1 (2C), 39.1, 39.7, 88.8, 107.6, 123.7 (2C), 124.1, 124.5, 125.4, 126.4, 128.1, 129.2, 134.7, 135.1, 135.6, 137.6, 142.3, 146.6, 159.6; HRMS (FAB) $m / z$ calcd for $\mathrm{C}_{31} \mathrm{H}_{53} \mathrm{OSi}(\mathrm{M}+\mathrm{H}) 469.3866$, found 469.3879 .

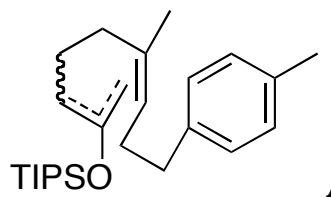

A Mixture (10a) of Triisopropyl $((2 Z, 6 Z)$ - and $(2 E, 6 Z)-6$-methyl-9-p-tolylnona2,6-dien-2-yloxy)silanes and (Z)-Triisopropyl(6-methyl-9-p-tolylnona-1,6-dien-2-yloxy)silane: $98 \%$ yield. (2E)-1-:(2Z)-2-:1-enyl 10a $=1: 30: 69$. Colorless oil; TLC, $R_{\mathrm{f}}=0.78$ (hexane-EtOAc $=4: 1$ ); IR (film) 2946, 2867, 1624, 1515, 1464, 1380, 1314, 1265, 1018, $883 \mathrm{~cm}^{-1}$; ${ }^{1} \mathrm{H}$ NMR $\left(\mathrm{CDCl}_{3}, 300 \mathrm{MHz}\right.$, note: all peaks could not be determined exactly.) $\delta 1.01-1.22(\mathrm{~m}, 21 \mathrm{H}), 1.50-1.60(\mathrm{~m}, 2 \mathrm{H}), 1.67(\mathrm{~s}, 3 \mathrm{H})$, $1.76(\mathrm{~s}, 3 \mathrm{H},(2 E)$-2-enyl isomer), $1.81(\mathrm{~s}, 3 \mathrm{H},(2 \mathrm{Z})$-2-enyl isomer), $2.01(\mathrm{t}, J=7.5 \mathrm{~Hz}, 4 \mathrm{H}), 2.10(\mathrm{t}, J=$ $7.2 \mathrm{~Hz}, 2 \mathrm{H},(2 \mathrm{Z})-2$ - and (2E)-2-enyl isomers), 2.22-2.32 (m, 2H), $2.32(\mathrm{~s}, 3 \mathrm{H}), 2.58(\mathrm{t}, J=7.2 \mathrm{~Hz}, 2 \mathrm{H})$, $3,98(\mathrm{~s}, 1 \mathrm{H}, 1$-enyl isomer), $4.01(\mathrm{~s}, 1 \mathrm{H}, 1$-enyl isomer), $4.30(\mathrm{t}, J=6.6 \mathrm{~Hz}, 1 \mathrm{H},(2 \mathrm{Z})$-2-enyl isomer), $4.61\left(\mathrm{t}, J=6.6 \mathrm{~Hz}, 1 \mathrm{H},(2 E)\right.$-2-enyl isomer), $5.18(\mathrm{t}, J=6.9 \mathrm{~Hz}, 1 \mathrm{H}), 7.08(\mathrm{~s}, 4 \mathrm{H}) ;{ }^{13} \mathrm{C} \mathrm{NMR}\left(\mathrm{CDCl}_{3}, 75\right.$ $\mathrm{MHz}$, note: all peaks could not be determined exactly.) $\delta 12.6$ (3C, TIPS), 13.2, 18.0 (6C, TIPS), 21.0, 22.0, 23.3, 23.8, 25.3, 30.0 (2C), 31.3, 32.9, 36.0, 36.6, 88.9, 107.5, 124.4, 124.6, 128.2 (2C), 128.9 (2C), 135.0, 135.7, 135.9, 139.2, 139.3, 146.7, 159.5; HRMS (FAB) $m / z$ calcd for $\mathrm{C}_{26} \mathrm{H}_{45} \mathrm{OSi}(\mathrm{M}+\mathrm{H})$ 401.3240, found, 401.3251 .

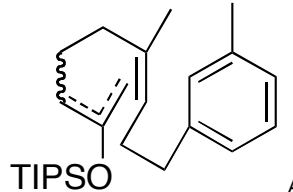

A Mixture (10b) of Triisopropyl((2Z,6Z)- and (2E,6Z)-6-methyl-9-m-tolylnona-2,6dien-2-yloxy)silanes and (Z)-Triisopropyl(6-methyl-9-p-tolylnona-1,6-dien-2-yloxy)silane: $>99 \%$ yield, (2E)-2-:(2Z)-2-:1-enyl 10b $=2: 26: 72$. Colorless oil; TLC, $R_{\mathrm{f}}=0.74$ (hexane-EtOAc $=4: 1$ ); IR (film) 2946, 2867, 1656, 1609, 1464, 1380, 1314, 1265, 1016, $883 \mathrm{~cm}^{-1} ;{ }^{1} \mathrm{H} \mathrm{NMR}\left(\mathrm{CDCl}_{3}, 300 \mathrm{MHz}\right.$, note: all peaks could not be determined exactly.) $\delta 1.03-1.22(\mathrm{~m}, 21 \mathrm{H}), 1.51-1.61(\mathrm{~m}, 2 \mathrm{H}), 1.68(\mathrm{~s}, 3 \mathrm{H})$, $1.76(\mathrm{~s}, 3 \mathrm{H},(2 E)-2$-enyl isomer), 1.81 (s, 3H, (2Z)-2-enyl isomer), $2.02(\mathrm{t}, J=7.5 \mathrm{~Hz}, 4 \mathrm{H}), 2.08-2.13$ $(\mathrm{m}, 2 \mathrm{H},(2 \mathrm{E})$-2-enyl and (2Z)-2-enyl isomers), 2.25-2.33 (m, 2H), $2.33(\mathrm{~s}, 3 \mathrm{H}), 2.58(\mathrm{t}, J=7.5 \mathrm{~Hz}, 2 \mathrm{H})$, $3,98(\mathrm{~s}, 1 \mathrm{H}, 1$-enyl isomer), $4.01(\mathrm{~s}, 1 \mathrm{H}, 1$-enyl isomer), 4.31 (t, $J=6.6 \mathrm{~Hz}, 1 \mathrm{H},(2 \mathrm{Z})-2$-enyl isomer), $4.61(\mathrm{t}, J=6.6 \mathrm{~Hz}, 1 \mathrm{H},(2 E)$-2-enyl isomer), 5.19 (t, $J=7.2 \mathrm{~Hz}, 1 \mathrm{H}), 6.98-7.00(\mathrm{~m}, 3 \mathrm{H}), 7.16$ (t, $J=7.2$ $\mathrm{Hz}, 1 \mathrm{H}) ;{ }^{13} \mathrm{C} \mathrm{NMR}\left(\mathrm{CDCl}_{3}, 75 \mathrm{MHz}\right.$, note: all peaks could not be determined exactly.) $\delta 12.6$ (3C, TIPS), 13.2, 18.0 (6C, TIPS), 21.4, 23.0, 23.3 (2 C), 23.8, 25.3, 29.9, 31.3, 31.3, 31.9, 36.3, 36.4, 36.6, 88.9, 107.5, 124.4, 124.6, 125.4, 126.4, 126.4, 128.1, 129.2, 135.7, 135.9, 137.6, 142.3, 146.7, 159.5; HRMS (FAB) $m / z$ calcd for $\mathrm{C}_{26} \mathrm{H}_{45} \mathrm{OSi}(\mathrm{M}+\mathrm{H})$ 401.3240, found 401.3236.

General Procedure for the Synthesis of tert-Butyldiphenylsilyl Enol Ethers 4, 7, and 10 from Ketones 34 (Step l, Scheme 4): To a AgOTf (257mg, $1.0 \mathrm{mmol})$ in $\mathrm{CH}_{2} \mathrm{Cl}_{2}(2.0 \mathrm{~mL})$ in a round bottom flask covered with aluminum foil was added TBDPSCl $(260 \mu \mathrm{L}, 1.0 \mathrm{mmol})$ and the resulting suspension was stirred for 2 days at room temperature under $\mathrm{N}_{2}$ atmosphere. The mixture was then added via cannula to a solution of $\mathbf{3 4}(0.50 \mathrm{mmol})$ in dry $\mathrm{CH}_{2} \mathrm{Cl}_{2}(1 \mathrm{~mL})$ was added $\mathrm{Et}_{3} \mathrm{~N}(209 \mu \mathrm{L}, 1.50 \mathrm{mmol})$ at $0{ }^{\circ} \mathrm{C}$. The resulting mixture was warmed to room temperature while being stirred for $12 \mathrm{~h}$. After the reaction was quenched with saturated $\mathrm{NaHCO}_{3}$ and the aqueous layer was extracted with $\mathrm{Et}_{2} \mathrm{O}$ (twice), the combined organic layers were dried over anhydrous $\mathrm{MgSO}_{4}$ and concentrated in vacuo. The residue was purified by flash chromatography on silica gel to give 4e, 7, or $\mathbf{1 0}$ as a colorless oil. The regio- and stereo-isomeric ratio ${ }^{16}$ of products was determined by ${ }^{1} \mathrm{H}$ NMR analysis. 


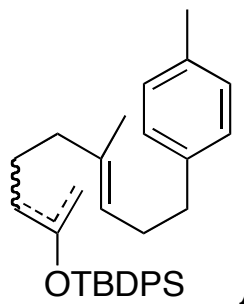

A Mixture (4e) of tert-Butyl((2Z,6E)- and $(2 E, 6 E)-6$-methyl-9-p-tolylnona-2,6-dien-2yloxy)diphenylsilane and $(E)$-tert-Butyl(6-methyl-9-p-tolylnona-1,6-dien-2-yloxy)diphenylsilane: $84 \%$ yield, $(2 E)$-2-enyl:(2Z)-2-enyl:1-enyl $\mathbf{4 e}=3: 37: 60$. Column chromatography (hexane-EtOAc $=$ 50:1); colorless oil; TLC, $R_{\mathrm{f}}=0.70$ (hexane-EtOAc $\left.=4: 1\right) ;{ }^{1} \mathrm{H} \mathrm{NMR}\left(\mathrm{CDCl}_{3}, 300 \mathrm{MHz}\right.$, note: all peaks could not be determined exactly.) $\delta 1.04$ (s, 9H, (2E)-2-enyl isomer), 1.07 (s, 9H, (2Z)-2-enyl isomer), 1.43 (s, 3H, (2Z)-2-enyl isomer), 1.50 (s, 3H, (2E)-2-enyl isomer), 1.56 (s, 3H), 1.69 (q, J = 7.5 Hz, 2H), 1.95-2.11 (m, 3H), 2.20-2.33 (m, 3H), $2.30(\mathrm{~s}, 3 \mathrm{H}), 2.60(\mathrm{t}, J=7.8 \mathrm{~Hz}, 2 \mathrm{H}), 3,71$ (s, 1H, 1-enyl isomer), 3.91 (s, 1H, 1-enyl isomer), 4.34 (t, $J=6.6 \mathrm{~Hz}, 1 \mathrm{H},(2 Z)$-2-enyl isomer), 4.50 (t, $J=7.5 \mathrm{~Hz}, 1 \mathrm{H},(2 E)$-2enyl isomer), 5.16-5.22 (m, 1H), $7.08(\mathrm{~s}, 4 \mathrm{H}), 7.34-7.44(\mathrm{~m}, 6 \mathrm{H}), 7.70-7.76(\mathrm{~m}, 4 \mathrm{H}) ;{ }^{13} \mathrm{C} \mathrm{NMR}\left(\mathrm{CDCl}_{3}\right.$, $75 \mathrm{MHz}$, note: all peaks could not be determined exactly.) $\delta 15.8,15.9,19.3,19.4,21.0,22.8,24.0,25.1$, $26.5,26.7,30.1,35.7,36.1,39.0,39.6,91.4,107.7,123.6,124.0,127.6,128.3,128.9,129.7$, 132.9, $134.3,135.3,135.3,135.5,135.8,139.3,147.0,158.8$.

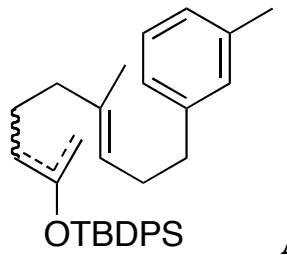

A Mixture (4f) of tert-Butyl $((2 Z, 6 E)$ - and $(2 E, 6 E)-6$-methyl-9-m-tolylnona-2,6-dien2-yloxy)diphenylsilanes and (E)-tert-Butyl(6-methyl-9-m-tolylnona-1,6-dien-2yloxy)diphenylsilane: $\quad 65 \%$ yield, $(2 E)$-2-enyl:(2Z)-2-enyl:1-enyl $\mathbf{4 f}=4: 34: 62$ Column chromatography (hexane-EtOAc $=50: 1$ to 20:1); colorless oil; TLC, $R_{\mathrm{f}}=0.70$ (hexane-EtOAc $=4: 1$ ); ${ }^{1} \mathrm{H} \mathrm{NMR}\left(\mathrm{CDCl}_{3}, 300 \mathrm{MHz}\right.$, note: all peaks could not be determined exactly.) $\delta 1.04$ (s, 9H, 1-enyl isomer), 1.06 (s, 9H, (2Z)-2-enyl isomer), 1.43 (s, 3H, (2Z)-2-enyl isomer), 1.50 (s, 3H, (2E)-2-enyl isomer), 1.62-1.78 (m, 2H), 1.94-2.12 (m, 3H), 2.14-2.32 (m, 3H), $2.32(\mathrm{~s}, 3 \mathrm{H}), 2.60(\mathrm{t}, J=7.5 \mathrm{~Hz}$, $2 \mathrm{H}), 3,71$ (s, 1H, 1-enyl isomer), $3.90(\mathrm{~s}, 1 \mathrm{H}, 1$-enyl isomer), 4.34 (t, $J=7.2 \mathrm{~Hz}, 1 \mathrm{H},(2 Z)$-2-enyl isomer), $4.51(\mathrm{t}, J=8.7 \mathrm{~Hz}, 1 \mathrm{H},(2 E)$-2-enyl isomer), $5.19(\mathrm{t}, J=7.2 \mathrm{~Hz}, 1 \mathrm{H}), 6.97-7.00(\mathrm{~m}, 3 \mathrm{H}), 7.18$ $(\mathrm{t}, J=7.2 \mathrm{~Hz}, 1 \mathrm{H}), 7.32-7.45(\mathrm{~m}, 6 \mathrm{H}), 7.69-7.76(\mathrm{~m}, 4 \mathrm{H}) ;{ }^{13} \mathrm{C} \mathrm{NMR}\left(\mathrm{CDCl}_{3}, 75 \mathrm{MHz}\right.$, note: all peaks could not be determined exactly.) $\delta 15.8,15.9,19.3,19.4,21.4,22.8,24.0,25.1,26.5,26.7,30.0,36.0$, $36.1(2 \mathrm{C}), 39.0,39.6,91.4,107.7,123.6,124.0,125.4,126.4(2 \mathrm{C}), 127.6,128.1,129.3,129.7,132.9$, $134.3,135.3,135.5(2 \mathrm{C}), 135.8,137.7,142.3,142.4,147.0,158.7$.

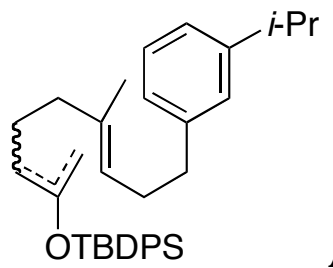
dien-2-yloxy)diphenylsilane and (E)-tert-Butyl(9-(3-isopropylphenyl)-6-methylnona-1,6-dien-2 yloxy)diphenylsilane: $\quad 93 \%$ yield, (2E)-2-enyl:(2Z)-2-enyl:1-enyl $\mathbf{4 h}=<1: 31: 69$. Column chromatography (hexane-EtOAc $=50: 1$ ); colorless oil; TLC, $R_{\mathrm{f}}=0.34$ (hexane-EtOAc $=25: 1$ ); IR (film) 2961, 1932, 2859, 2360, 1630, 1429, $1112 \mathrm{~cm}^{-1} ;{ }^{1} \mathrm{H} \mathrm{NMR}\left(\mathrm{CDCl}_{3}, 300 \mathrm{MHz}\right.$, note: all peaks could not be determined exactly.) $\delta 1.03$ (s, 9H, 1-enyl isomer), 1.06 (s, 9H, (2Z)-2-enyl isomer), 1.24 $(\mathrm{d}, J=6.9 \mathrm{~Hz}, 6 \mathrm{H}), 1.50(\mathrm{~s}, 3 \mathrm{H},(2 \mathrm{E})$-2-enyl isomer), $1.54(\mathrm{~s}, 3 \mathrm{H}), 1.62-1.72(\mathrm{~m}, 2 \mathrm{H}, 1$-enyl isomer), 1.94-2.35 (m, 6H), $2.62(\mathrm{t}, J=7.8 \mathrm{~Hz}, 2 \mathrm{H}), 2.80-2.94(\mathrm{~m}, 1 \mathrm{H}), 3,70$ (s, 1H, 1-enyl isomer), $3.90(\mathrm{~s}, 1 \mathrm{H}$, 1-enyl isomer), $4.34(\mathrm{t}, J=7.2 \mathrm{~Hz}, 1 \mathrm{H},(2 \mathrm{Z})$-2-enyl isomer), $5.20(\mathrm{t}, J=6.6 \mathrm{~Hz}, 1 \mathrm{H}), 7.00-7.05(\mathrm{~m}, 3 \mathrm{H})$, 7.18-7.23 (m, 1H), 7.35-7.46 (m, 6H), 7.71-7.75 (m, 4H); ${ }^{13} \mathrm{C} \mathrm{NMR}\left(\mathrm{CDCl}_{3}, 75 \mathrm{MHz}\right.$, note: all peaks could not be determined exactly.) $\delta 15.8,15.9,19.3,19.4,22.8,24.1,25.1,26.5,26.7,30.0,34.1,36.1$, 
36.2, 36.3, 39.0, 39.6, 91.5, 107.7, 123.7 (2C), 124.0, 125.9, 126.6, 127.5, 127.6, 125.2, 129.7, 133.0, $134.3,135.3,135.4,135.5,135.8,142.3,142.4,147.0,148.7,158.6$; HRMS (FAB) $\mathrm{m} / z$ calcd for $\mathrm{C}_{35} \mathrm{H}_{47} \mathrm{OSi}(\mathrm{M}+\mathrm{H})$ 511.3396, found 511.3380.

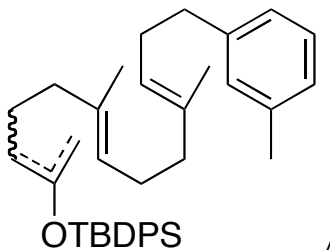

A Mixture $(7 \mathrm{c})$ of tert-Butyl $((2 E, 6 E, 10 E)$ - and $(2 Z, 6 E, 10 E)-6,10$-dimethyl-13-mtolyltrideca-2,6,10-trien-2-yloxy)diphenylsilanes and tert-Butyl ((6E,10E)-6,10-dimethyl-13-mtolyltrideca-1,6,10-trien-2-yloxy)diphenylsilane: $81 \%$ yield, (2E)-2-:(2Z)-2-:1-enyl 7c = 3:26:11. Column chromatography (hexane-EtOAc $=50: 1$ ); colorless oil; TLC, $R_{\mathrm{f}}=0.76$ (hexane-EtOAc $=4: 1$ ); ${ }^{1} \mathrm{H}$ NMR $\left(\mathrm{CDCl}_{3}, 300 \mathrm{MHz}\right.$, note: all peaks could not be determined exactly.) $\delta 1.04$ (s, 9H, 1-enyl isomer), $1.06(\mathrm{~s}, 9 \mathrm{H},(2 \mathrm{Z})$-enyl isomer), $1.47(\mathrm{~s}, 3 \mathrm{H},(2 \mathrm{Z})$-enyl isomer), $1.50(\mathrm{~s}, 3 \mathrm{H}$, (2E)-enyl isomer), $1.57(\mathrm{~s}, 3 \mathrm{H}), 1.60(\mathrm{~s}, 3 \mathrm{H}), 1.64-1.75(\mathrm{~m}, 2 \mathrm{H}), 1.94-2.12(\mathrm{~m}, 8 \mathrm{H}), 2.21-2.31(\mathrm{~m}, 2 \mathrm{H}), 2.31(\mathrm{~S}, 3 \mathrm{H}), 2.59$ $(\mathrm{t}, J=7.8 \mathrm{~Hz}, 2 \mathrm{H}), 3,72(\mathrm{~s}, 1 \mathrm{H}, 1$-enyl isomer), $3.92(\mathrm{~s}, 1 \mathrm{H}, 1$-enyl isomer), $4.35(\mathrm{t}, J=6.9 \mathrm{~Hz}, 1 \mathrm{H}$, (2Z)-enyl isomer), $4.51(\mathrm{t}, J=6.6 \mathrm{~Hz}, 1 \mathrm{H},(2 E)$-enyl isomer), $5.13(\mathrm{t}, J=6.6 \mathrm{~Hz}, 1 \mathrm{H}), 5.20(\mathrm{t}, J=6.9 \mathrm{~Hz}$, $1 \mathrm{H}), 6.97-6.99(\mathrm{~m}, 3 \mathrm{H}), 7.15(\mathrm{t}, J=752 \mathrm{~Hz}, 1 \mathrm{H}), 7.34-7.43(\mathrm{~m}, 6 \mathrm{H}), 7.69-7.76(\mathrm{~m}, 4 \mathrm{H}) ;{ }^{13} \mathrm{C} \mathrm{NMR}$ $\left(\mathrm{CDCl}_{3}, 75 \mathrm{MHz}\right.$, note: all peaks could not be determined exactly.) $\delta \mathrm{15.8}, 15.9,16.0$ (2C), 19.3, 19.4, 21.4, 22.8, 24.1, 25.1, 26.5, 26.6, 30.0, 36.0, 39.0, 39.7 (2C), 39.8, 65.8, 91.5, 107.8, 123.7 82C), 124.1, $124.5,125.4,126.4,127.6,128.1,129.2,129.7,132.9,134.3,134.7,135.1,135.3,135.4,135.5,135.6$, $137.6,142.3,146.9,158.6$.

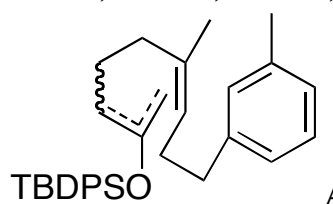

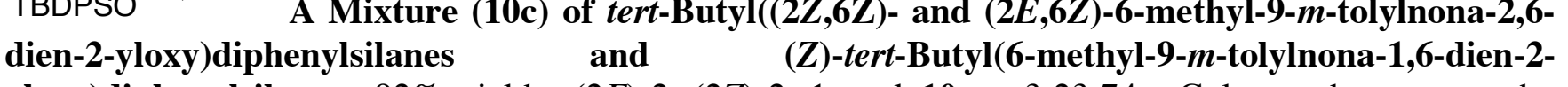
yloxy)diphenylsilane: $92 \%$ yield. $(2 E)-2-:(2 Z)-2-: 1-$ enyl 10c $=3: 23: 74$. Column chromatography (hexane-EtOAc $=50: 1)$; colorless oil; TLC, $R_{\mathrm{f}}=0.76$ (hexane-EtOAc $\left.=4: 1\right) ;{ }^{1} \mathrm{H} \mathrm{NMR}\left(\mathrm{CDCl}_{3}, 300\right.$ $\mathrm{MHz}$, note: all peaks could not be determined exactly.) $\delta 1.04$ (s, 9H, 1-enyl isomer), 1.06 (s, 9H, (2Z)2-enyl isomer), $1.50(\mathrm{~s}, 3 \mathrm{H},(2 \mathrm{Z})$-2-enyl isomer), $1.54(\mathrm{~s}, 3 \mathrm{H},(2 \mathrm{E})$-2-enyl isomer), 1.65-1.75 (m, 2H), $1.70(\mathrm{~s}, 3 \mathrm{H}), 2.08(\mathrm{q}, J=7.5 \mathrm{~Hz}, 4 \mathrm{H}), 2.20-2.33(\mathrm{~m}, 2 \mathrm{H}), 2.29(\mathrm{~s}, 3 \mathrm{H}), 2.56(\mathrm{t}, J=8.7 \mathrm{~Hz}, 2 \mathrm{H}), 3,74(\mathrm{~s}$, $1 \mathrm{H}, 1$-enyl isomer), 3.92 (s, 1H, 1-enyl isomer), $4.35(\mathrm{t}, J=6.6 \mathrm{~Hz}, 1 \mathrm{H},(2 \mathrm{Z})$-2-enyl isomer), $4.51(\mathrm{t}, J=$ $6.6 \mathrm{~Hz}, 1 \mathrm{H},(2 E)-2$-enyl isomer), $5.21(\mathrm{t}, J=6.6 \mathrm{~Hz}, 1 \mathrm{H}), 6.96-6.98(\mathrm{~m}, 3 \mathrm{H}), 7.14(\mathrm{t}, J=7.2 \mathrm{~Hz}, 1 \mathrm{H})$, 7.31-7.40 (m, 6H), 7.70-7.76 (m, 4H); ${ }^{13} \mathrm{C} \mathrm{NMR}\left(\mathrm{CDCl}_{3}, 75 \mathrm{MHz}\right.$, note: all peaks could not be determined exactly.) $\delta 19.3,19.4,21.4,22.9,23.3,23.4,23.8,25.2,26.5,26.7,29.9,31.3,31.8,36.3$, $36.4,91.5,107.7,124.5,124.8,125.5,126.4$ (2C), 127.6, 127.7, 128.1, 129.2, 129.7, 132.9, 134.2, 135.3, $135.4,135.5,135.8,137.6,142.2,142.3,147.8,158.5$.

General Procedure for the Synthesis of Triisopropylsilyl Enol Ethers 14 from Aldehydes 13 (Step m, Scheme 4): To a solution of 13 (1.17 mmol) in dry $\mathrm{CH}_{2} \mathrm{Cl}_{2}-\mathrm{Et}_{3} \mathrm{~N}(1: 1 \mathrm{v} / \mathrm{v}, 4 \mathrm{~mL})$ was added TIPSOTf $(338 \mu \mathrm{L}, 1.30 \mathrm{mmol})$ dropwise at $-78{ }^{\circ} \mathrm{C}$. The mixture was stirred for $2 \mathrm{~h}$ while the temperature was allowed to warm to $0{ }^{\circ} \mathrm{C}$. After the reaction was quenched with saturated $\mathrm{NaHCO}_{3}$, the aqueous layer was extracted with $\mathrm{Et}_{2} \mathrm{O}$ (twice). The combined organic layers were dried over anhydrous $\mathrm{MgSO}_{4}$ and concentrated in vacuo. The $(1 E) /(1 Z)$-isomeric ratio ${ }^{17}$ of crude products 14 was determined to be $\left\langle 5:>95\right.$ by ${ }^{1} \mathrm{H}$ NMR analysis. The residue was purified by flash chromatography $\left(\mathrm{SiO}_{2}\right.$, hexane $\rightarrow$ hexane:EtOAc $=100: 1)$ to give $(1 Z)-\mathbf{1 4}(>99 \% 1 Z)$ as a colorless oil. (1E)-14 was partially obtained by flash chromatography. 


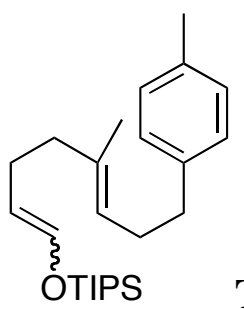
$>99 \%$ 1Z. TLC, $R_{\mathrm{f}}=0.71$ (hexane-EtOAc $\left.=4: 1\right), 0.33$ (hexane); ${ }^{1} \mathrm{H}$ NMR $\left(\mathrm{CDCl}_{3}, 300 \mathrm{MHz}\right) \delta 1.03-$ $1.19(\mathrm{~m}, 21 \mathrm{H}), 1.56(\mathrm{~s}, 3 \mathrm{H}), 2.00(\mathrm{t}, J=7.5 \mathrm{~Hz}, 1 \mathrm{H}), 2.16-2.32(\mathrm{~m}, 4 \mathrm{H}), 2.32(\mathrm{~s}, 3 \mathrm{H}), 2.59$ (dd, $J=6.9$, $8.7 \mathrm{~Hz}, 2 \mathrm{H}), 4.36(\mathrm{td}, J=5.7,7.0 \mathrm{~Hz}, 1 \mathrm{H}), 5.19(\mathrm{tm}, J=7.2 \mathrm{~Hz}, 1 \mathrm{H}), 6.26(\mathrm{dt}, J=1.5,5.7 \mathrm{~Hz}, 1 \mathrm{H}), 7.08$ (s, 4H); ${ }^{13} \mathrm{C}$ NMR $\left(\mathrm{CDCl}_{3}, 75 \mathrm{MHz}\right) \delta 12.0$ (3C, TIPS), 15.8, 17.7 (6C, TIPS), 21.0, 22.3, 30.1, 35.7, $39.6,109.5,123.7,128.3,128.9,135.0,135.7,138.9,139.4$.

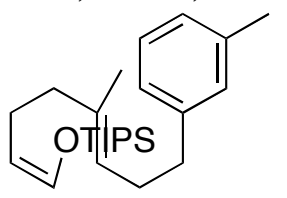

Triisopropyl((1Z,5E)-5-methyl-8-m-tolylocta-1,5-dienyloxy)silane $\quad(14 b): \quad>95 \%$ yield. $>99 \%$ 1Z. Column chromatography (hexane); colorless oil; TLC, $R_{\mathrm{f}}=0.74$ (hexane-EtOAc $=$ 4:1), $R_{\mathrm{f}}=0.17$ (hexane); ${ }^{1} \mathrm{H}$ NMR $\left(\mathrm{CDCl}_{3}, 300 \mathrm{MHz}\right) \delta 1.03-1.19(\mathrm{~m}, 21 \mathrm{H}), 1.57(\mathrm{~s}, 3 \mathrm{H}), 2.04(\mathrm{t}, J=7.6$ $\mathrm{Hz}, 2 \mathrm{H}), 2.17-2.33(\mathrm{~m}, 4 \mathrm{H}), 2.33(\mathrm{~s}, 3 \mathrm{H}), 2.59(\mathrm{t}, J=7.8 \mathrm{~Hz}, 2 \mathrm{H}), 4.37(\mathrm{td}, J=5.7,6.4 \mathrm{~Hz}, 1 \mathrm{H}), 5.20$ (tm, $J=6.9 \mathrm{~Hz}, 1 \mathrm{H}), 6.26(\mathrm{dt}, J=1.2,5.7 \mathrm{~Hz}, 1 \mathrm{H}), 6.98-7.00(\mathrm{~m}, 3 \mathrm{H}), 7.16(\mathrm{t}, J=7.5 \mathrm{~Hz}, 1 \mathrm{H})$.

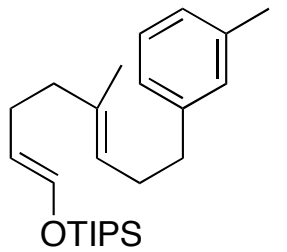

Triisopropyl((1E,5E)-5-methyl-8-m-tolylocta-1,5-dienyloxy)silane $\quad(14 b): \quad 14 b$ $((1 E):(1 Z)=76: 24)$ was partially obtained by flash chromatography $\left(\mathrm{SiO}_{2}\right.$, hexane:EtOAc $\left.=100: 1\right)$. Colorless oil; TLC, $R_{\mathrm{f}}=0.74$ (hexane-EtOAc $\left.=4: 1\right), 0.17$ (hexane); ${ }^{1} \mathrm{H}$ NMR $\left(\mathrm{CDCl}_{3}, 300 \mathrm{MHz}\right) \delta$ $1.03-1.20(\mathrm{~m}, 21 \mathrm{H}), 1.55$ (s, 3H), 1.90-2.10 (brs, $4 \mathrm{H}), 2.23-2.33$ (m, 2H), 2.33 (s, 3H), 2.59 (t, $J=8.1$ $\mathrm{Hz}, 2 \mathrm{H}), 4.94-5.02(\mathrm{~m}, 1 \mathrm{H}), 5.19(\mathrm{t}, J=6.9 \mathrm{~Hz}, 1 \mathrm{H}), 6.31(\mathrm{~d}, J=12.0 \mathrm{~Hz}, 1 \mathrm{H}), 6.98-7.00(\mathrm{~m}, 3 \mathrm{H}), 7.17$ $(\mathrm{t}, J=7.6 \mathrm{~Hz}, 1 \mathrm{H})$.

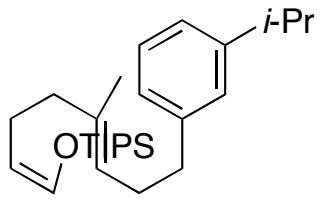

Triisopropyl((1Z,5E)-8-(3-isopropylphenyl)-5-methylocta-1,5-dienyloxy)silane (14c): $>95 \%$ yield. $>99 \% 1 Z$. Column chromatography (hexane); colorless oil; TLC, $R_{\mathrm{f}}=0.70$ (hexane-EtOAc = 4:1), 0.22 (hexane); IR (film) 2961, 2946, 2868, 1652, 1464, 1118. $883 \mathrm{~cm}^{-1} ;{ }^{1} \mathrm{H}$ NMR $\left(\mathrm{CDCl}_{3}, 300 \mathrm{MHz}\right) \delta 1.03-1.19(\mathrm{~m}, 21 \mathrm{H}), 1.24(\mathrm{~d}, J=6.9 \mathrm{~Hz}, 6 \mathrm{H}), 1.56(\mathrm{~s}, 3 \mathrm{H}), 2.01(\mathrm{t}, J=7.5$ $\mathrm{Hz}, 2 \mathrm{H}), 2.17-2.33(\mathrm{~m}, 4 \mathrm{H}), 2.62(\mathrm{t}, J=7.8 \mathrm{~Hz}, 2 \mathrm{H}), 2.80-2.94(\mathrm{~m}, 1 \mathrm{H}), 4.36(\mathrm{dd}, J=6.9,12.9 \mathrm{~Hz}, 1 \mathrm{H})$, $5.21(\mathrm{dt}, J=0.9,6.9 \mathrm{~Hz}, 1 \mathrm{H}), 6.25(\mathrm{brd}, J=5.7 \mathrm{~Hz}, 1 \mathrm{H}), 7.00-7.05(\mathrm{~s}, 3 \mathrm{H}), 7.17-7.23(\mathrm{~m}, 1 \mathrm{H}) ;{ }^{13} \mathrm{C}$ NMR $\left(\mathrm{CDCl}_{3}, 75 \mathrm{MHz}\right) \delta 11.9$ (3C, TIPS), 15.8, 17.7 (6C, TIPS), 22.2, 24.0 (2C), 30.1, 34.1, 36.2, 39.6, 109.4, 123.6, 123.7, 125.9, 126.6, 128.1, 135.7, 138.9, 142.4, 148.7; HRMS (FAB) $\mathrm{m} / \mathrm{z}$ calcd for $\mathrm{C}_{27} \mathrm{H}_{46} \mathrm{OSiNa}(\mathrm{M}+\mathrm{Na})$ 437.3216, found 437.3205.

\section{Preparation of 1-(Chloromethyl)-3-isopropylbenzene (39)}

1-(Chloromethyl)-3-isopropylbenzene (39), which was a starting material of Butsugan coupling to synthesize $\mathbf{4 h}$ and $\mathbf{1 4 c}$, was prepared as shown in Scheme 4. 
Scheme 4. Preparation of $\mathbf{3 9}^{a}$

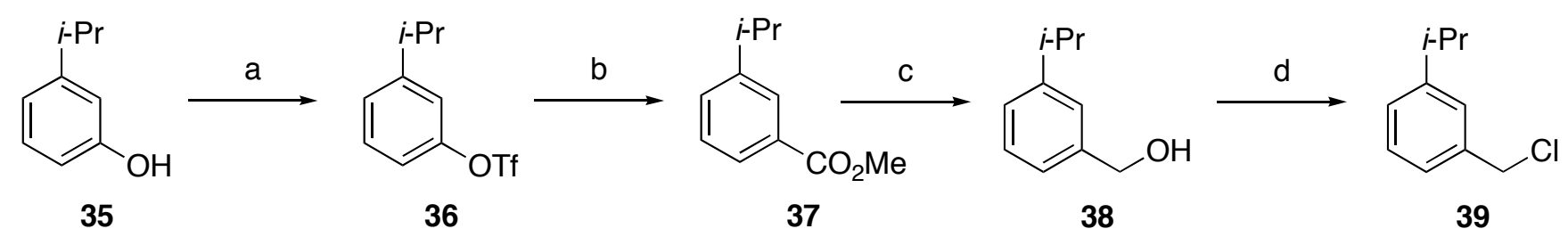

a Reagents and conditions: a) $\mathrm{Tf}_{2} \mathrm{O}$, pyridine, $\mathrm{ClCH}_{2} \mathrm{CH}_{2} \mathrm{Cl}, 0{ }^{\circ} \mathrm{C}$ to rt, $1 \mathrm{~h}$; b) $\mathrm{Pd}(\mathrm{OAc})_{2}$, dppp, $i-\mathrm{Pr}_{2} \mathrm{NEt}$, $\mathrm{MeOH}, \mathrm{CO}, \mathrm{DMSO}, 80^{\circ} \mathrm{C}, 12 \mathrm{~h}$; c) $\mathrm{LiAlH}_{4}, \mathrm{THF}, 0^{\circ} \mathrm{C}, 1 \mathrm{~h}$; d) $\mathrm{SO}_{2} \mathrm{Cl}$, pyridine, benzene, reflux, $5 \mathrm{~h}$.<smiles>CCCc1cccc(Cl)c1</smiles>

OTf 3-Isopropylphenyl Trifluoromethanesulfonate (36) (Step a, Scheme 4): To a solution of $35(4.11 \mathrm{~mL}, 30.0 \mathrm{mmol})$ in dichloroethane $(30 \mathrm{~mL})$ was added pyridine $(4.81 \mathrm{~mL}, 60.0 \mathrm{mmol})$ and $\mathrm{Tf}_{2} \mathrm{O}(10.1 \mathrm{~mL}, 60.0 \mathrm{mmol})$ at $0{ }^{\circ} \mathrm{C}$. After stirring for $1 \mathrm{~h}$ at room temperature, the reaction mixture was poured into brine, washed with $\mathrm{H}_{2} \mathrm{O}$ (twice), and extracted with $\mathrm{Et}_{2} \mathrm{O}$ (twice). Solvents were removed in vacuo and the crude oil was distilled $\left(50-55^{\circ} \mathrm{C}, 1\right.$ torr) to give $36(7.73 \mathrm{~g}, 28.8 \mathrm{mmol})$ as a colorless oil in $96 \%$ yield. TLC, $R_{\mathrm{f}}=0.65$ (hexane-EtOAc $\left.=4: 1\right) ;{ }^{1} \mathrm{H} \mathrm{NMR}\left(\mathrm{CDCl}_{3}, 300 \mathrm{MHz}\right) \delta 1.26(\mathrm{~d}, J=6.9 \mathrm{~Hz}$, $6 \mathrm{H}), 2.89-3.02(\mathrm{~m}, 1 \mathrm{H}), 7.07-7.12(\mathrm{~m}, 2 \mathrm{H}), 7.24-7.27(\mathrm{~m}, 1 \mathrm{H}), 7.36(\mathrm{t}, J=7.8 \mathrm{~Hz}, 1 \mathrm{H})$; ${ }^{13} \mathrm{C} \mathrm{NMR}$ $\left(\mathrm{CDCl}_{3}, 75 \mathrm{MHz}\right) \delta 23.6(2 \mathrm{C}), 33.9,118.5,118.8$ (q, $\left.J_{\mathrm{CF}}=319 \mathrm{~Hz}\right), 119.3,126.5,130.0,149.7,151.9$; ${ }^{19} \mathrm{~F} \mathrm{NMR}\left(\mathrm{CDCl}_{3}, 282 \mathrm{MHz}\right) \delta-73.7(\mathrm{~s}, 3 \mathrm{~F})$.<smiles>CCCc1cccc(Cl)c1</smiles>

$\mathrm{CO}_{2} \mathrm{Me}$ Methyl 3-Isopropylbenzoate (37) (Step b, Scheme 5): 36 (6.71g, $25.0 \mathrm{mmol}$ ), $\mathrm{Pd}(\mathrm{OAc})_{2}(853 \mathrm{mg}, 3.80 \mathrm{mmol})$, dppp (1.57 g, $\left.3.80 \mathrm{mmol}\right), i$-Pr $2 \mathrm{NEt}(9.6 \mathrm{~mL}, 55.0 \mathrm{mmol})$ and $\mathrm{MeOH}$ $(25 \mathrm{~mL}, 625 \mathrm{mmol})$ in DMSO $(100 \mathrm{~mL})$ was sealed and quickly cycled three times between open-air (in draft) and $\mathrm{CO}$ atmosphere. The $\mathrm{CO}$ pressure in the flask was increased to ca. 1.5 atm and partially immersed in an $80^{\circ} \mathrm{C}$ oil bath. After $12 \mathrm{~h}$, the mixture was cooled to room temperature and poured into brine, extracted with $\mathrm{Et}_{2} \mathrm{O}$ (twice), washed with brine (twice) and water (3 times). The combined organic layers were dried over anhydrous $\mathrm{MgSO}_{4}$ and concentrated in vacuo. The residue was passed a short pad of silica gel to give 37, which was used for next step without further purification. TLC, $R_{\mathrm{f}}=$ 0.62 (hexane-EtOAc = 4:1); ${ }^{1} \mathrm{H} \mathrm{NMR}\left(\mathrm{CDCl}_{3}, 300 \mathrm{MHz}\right) \delta 1.27(\mathrm{~d}, J=6.9 \mathrm{~Hz}, 6 \mathrm{H}), 2.89-3.04(\mathrm{~m}, 1 \mathrm{H})$, $3.92(\mathrm{~s}, 3 \mathrm{H}), 7.36(\mathrm{t}, J=7.5 \mathrm{~Hz}, 1 \mathrm{H}), 7.43(\mathrm{dt}, J=1.5,7.5 \mathrm{~Hz}, 1 \mathrm{H}), 8.76(\mathrm{dt}, J=1.5,6.0 \mathrm{~Hz}, 1 \mathrm{H}), 7.91$ $(\mathrm{t}, J=1.5 \mathrm{~Hz}, 1 \mathrm{H})$.<smiles>CCCc1cccc(CO)c1</smiles>

(3-Isopropylphenyl)methanol (38) (Step c, Scheme 4): To a solution of 37 (1.10 g, 6.15 mmol $)$ in THF $(20 \mathrm{~mL})$ was added $\mathrm{LiAlH}_{4}(350 \mathrm{mg}, 9.23 \mathrm{mmol})$ slowly at $0{ }^{\circ} \mathrm{C}$. After stirring for $1 \mathrm{~h}$ at $0{ }^{\circ} \mathrm{C}$, excess of $\mathrm{LiAlH}_{4}$ was decomposed with EtOAc and $\mathrm{H}_{2} \mathrm{O}$. After that $\mathrm{Na}_{2} \mathrm{SO}_{4}$ was added and stirred for $1-2 \mathrm{~h}$ at room temperature. The resulting mixture was through celite and the solvent evaporated under vacuum. The residue was purified by flash chromatography on silica gel eluting with hexane$\mathrm{EtOAc}=5: 1$ to give $38(896 \mathrm{mg}, 5.97 \mathrm{mmol}, 97 \%$ for 2 steps $)$ as a colorless oil. TLC, $R_{\mathrm{f}}=0.62$ (hexane-EtOAc $=4: 1) ;{ }^{1} \mathrm{H}$ NMR $\left(\mathrm{CDCl}_{3}, 300 \mathrm{MHz}\right) \delta 1.25(\mathrm{~d}, J=6.9 \mathrm{~Hz}, 6 \mathrm{H}), 1.85-1.94(\mathrm{~m}, \mathrm{OH})$, 2.84-2.98 (m, 1H), $4.65(\mathrm{~d}, J=5.7 \mathrm{~Hz}, 2 \mathrm{H}), 7.15-7.31(\mathrm{~m}, 4 \mathrm{H}) ;{ }^{13} \mathrm{C} \mathrm{NMR}\left(\mathrm{CDCl}_{3}, 75 \mathrm{MHz}\right) \delta 23.8(2 \mathrm{C})$, 
$33.9,64.7,124.3,124.9,125.3,128.2,140.7,148.8$.

$\mathrm{Cl}_{1}^{i-\mathrm{Pr}}$

1-(Chloromethyl)-3-isopropylbenzene (39) (Step d, Scheme 4): To a solution of 38 (793

$\mathrm{mg}, 5.28 \mathrm{mmol}$ ) in benzene $(6 \mathrm{~mL})$ was added pyridine (one drop) and $\mathrm{SOCl}_{2}(616 \mu \mathrm{L}, 8.45 \mathrm{mmol})$ dropwise at $\mathrm{rt}$ and the resulting mixture was heated to reflux at $80^{\circ} \mathrm{C}$. After stirring for $5 \mathrm{~h}$, the solution was cooled and concentrated under vacuum. The residue was extracted with $\mathrm{Et}_{2} \mathrm{O}$, and washed with water and brine. The combined organic layers were dried over anhydrous $\mathrm{MgSO}_{4}$ and concentrated in vacuo and the residue was purified by short column chromatography on silica gel eluting with hexane to give $39(821 \mathrm{mg}, 4.87 \mathrm{mmol}, 92 \%)$ as a colorless oil. TLC, $R_{\mathrm{f}}=0.48$ (hexane-EtOAc $\left.=25: 1\right) ;{ }^{1} \mathrm{H}$ NMR $\left(\mathrm{CDCl}_{3}, 300 \mathrm{MHz}\right) \delta 1.25(\mathrm{~d}, J=7.2 \mathrm{~Hz}, 6 \mathrm{H}), 2.85-2.99(\mathrm{~m}, 1 \mathrm{H}), 4.59(\mathrm{~s}, 2 \mathrm{H}), 7.17-7.32(\mathrm{~m}, 4 \mathrm{H}) ;{ }^{13} \mathrm{C}$ $\mathrm{NMR}\left(\mathrm{CDCl}_{3}, 75 \mathrm{MHz}\right) \delta 23.8$ (2C), 33.9, 42.3, 125.9, 126.3, 126.6, 128.6, 137.3, 149.2.

General Procedure for the $\mathrm{SnCl}_{4}$-Promoted Cyclization (Tables 1-4 and Scheme 2): To a flame-dried schlenk charged with toluene or $\mathrm{CH}_{2} \mathrm{Cl}_{2}(2 \mathrm{~mL})$ was added a $0.1 \mathrm{M}$ solution of $\mathrm{SnCl}_{4}$ in hexane or $\mathrm{CH}_{2} \mathrm{Cl}_{2}(100 \mu \mathrm{L}, 10 \mu \mathrm{mol})$, and the mixture was cooled to $-78^{\circ} \mathrm{C}$. To this solution was added a $1.0 \mathrm{M}$ solution of starting material $(\mathbf{1}, 4,7, \mathbf{1 0}, \mathbf{1 3}, \mathbf{1 4})$ in toluene or $\mathrm{CH}_{2} \mathrm{Cl}_{2}(100 \mu \mathrm{L}, 0.1 \mathrm{mmol})$ dropwise over $1 \mathrm{~min}$. The reaction mixture was quenched with saturated aqueous $\mathrm{NaHCO}_{3}$, and the aqueous phase was extracted with ether. The combined organic layers were dried over anhydrous $\mathrm{MgSO}_{4}$ and concentrated in vacuo. The crude product was analyzed directly or purified by column chromatography on silica gel to give cyclic compounds.

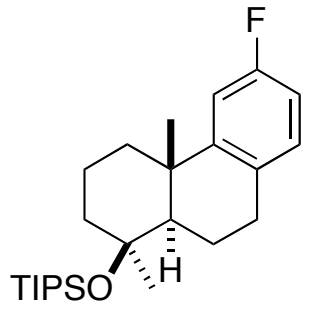

$\left(1 S^{*}, 4 \mathrm{a} S *, 10 \mathrm{a} R *\right)$-6-Fluoro-1,4a-dimethyl-1,2,3,4,4a,9,10,10a-octahydrophenanthren-1-yloxy)triisopropylsilane (5aß) (Entry 1, Table 1): Column chromatography (hexane); amorphous solid; TLC, $R_{\mathrm{f}}=0.76$ (hexane-EtOAc $=4: 1$ ), 0.50 (hexane); IR (film) 2945, 2868, 1496, 1465, 1051, $883 \mathrm{~cm}^{-1} ;{ }^{1} \mathrm{H}$ NMR $\left(\mathrm{CDCl}_{3}, 500 \mathrm{MHz}\right) \delta 1.09-1.13(\mathrm{~m}, 21 \mathrm{H}), 1.19-1.45(\mathrm{~m}, 3 \mathrm{H})$, $1.29(\mathrm{~s}, 3 \mathrm{H}), 1.33(\mathrm{~s}, 3 \mathrm{H}), 1.53-1.59(\mathrm{~m}, 1 \mathrm{H}), 1.86-1.89(\mathrm{~m}, 1 \mathrm{H}), 1.90-2.07(\mathrm{~m}, 3 \mathrm{H}), 2.18-2.21(\mathrm{~m}, 1 \mathrm{H})$, $2.78-2.85(\mathrm{~m}, 1 \mathrm{H}), 2.95(\mathrm{dd}, J=7.0,17.0 \mathrm{~Hz}, 1 \mathrm{H}), 6.76(\mathrm{dt}, J=2.5,8.5 \mathrm{~Hz}, 1 \mathrm{H}), 6.93(\mathrm{dd}, J=2.5,11.0$ $\mathrm{Hz}, 1 \mathrm{H}), 6.98(\mathrm{dd}, J=6.0,8.5 \mathrm{~Hz}, 1 \mathrm{H}) ;{ }^{13} \mathrm{C} \mathrm{NMR}\left(\mathrm{CDCl}_{3}, 125 \mathrm{MHz}\right) \delta 14.1$ (3C), $18.4(3 \mathrm{C}), 18.5,18.6$ (3C), 18.8, 24.7, 28.8, 30.3, 37.7, 38.5, 41.3, 50.5, 74.9, 110.6, 112.0, 130.0, 130.5, 151.9, 161.2 (d, $J_{\mathrm{CF}}$ $=240 \mathrm{~Hz}) ;{ }^{19} \mathrm{~F} \mathrm{NMR}\left(\mathrm{CDCl}_{3}, 282 \mathrm{MHz}\right) \delta-118.5(\mathrm{dd}, J=9.0,18.0 \mathrm{~Hz}) ; \mathrm{HRMS}(\mathrm{EI}) \mathrm{m} / z$ calcd for $\mathrm{C}_{22} \mathrm{H}_{34} \mathrm{FOSi}(\mathrm{M}-i$-Pr) 361.2363, found 361.2355. No detectable amounts of 5a $\mathbf{\alpha}$ and $\mathbf{6 a}$ were obtained.<smiles>CC12CCC[C@@](C)(OS(=O)(F)(F)F)[C@@H]1CCc1ccccc12</smiles>

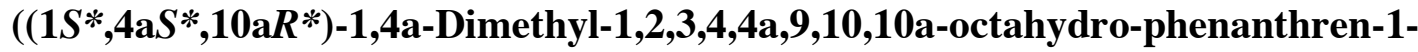
yloxy)triisopropylsilane $(\mathbf{5 b} \boldsymbol{\beta})$ (Entry 2, Table 1): Column chromatography (hexane); amorphous solid; TLC, $R_{\mathrm{f}}=0.76$ (hexane-EtOAc = 4:1), 0.33 (hexane); IR (film) 2945, 2868, 1464, 1051, 993, 883 $\mathrm{cm}^{-1} ;{ }^{1} \mathrm{H}$ NMR $\left(\mathrm{CDCl}_{3}, 500 \mathrm{MHz}\right) \delta 1.04-1.16(\mathrm{~m}, 21 \mathrm{H}), 1.26-1.40(\mathrm{~m}, 2 \mathrm{H}), 1.31(\mathrm{~s}, 3 \mathrm{H}), 1.33(\mathrm{~s}, 3 \mathrm{H})$, $1.44(\mathrm{dt}, J=3.5,13.0 \mathrm{~Hz}, 1 \mathrm{H}), 1.53-1.59(\mathrm{~m}, 1 \mathrm{H}), 1.88(\mathrm{dd}, J=1.5,14.0 \mathrm{~Hz}, 1 \mathrm{H}), 1.95-2.08(\mathrm{~m}, 3 \mathrm{H})$, $2.30(\mathrm{dm}, J=12.5 \mathrm{~Hz}, 1 \mathrm{H}), 2.84-2.91(\mathrm{~m}, 1 \mathrm{H}), 2.98(\mathrm{ddd}, J=2.5,7.0,17.6 \mathrm{~Hz}, 1 \mathrm{H}), 7.04-7.08(\mathrm{~s}, 2 \mathrm{H})$, $7.11(\mathrm{dt}, J=12.0,17.0 \mathrm{~Hz}, 1 \mathrm{H}), 7.25-7.26(\mathrm{~m}, 1 \mathrm{H}) ;{ }^{13} \mathrm{C} \mathrm{NMR}\left(\mathrm{CDCl}_{3}, 125 \mathrm{MHz}\right) \delta 14.1(3 \mathrm{C}), 18.4(3 \mathrm{C})$, 
18.56 (3C), 18.62, 18.9, 24.8, 29.6, 30.3, 37.5, 38.6, 41.4, 50.8, 75.0, 124.1, 125.1, 125.5, 128.9, 135.2, 149.8; HRMS (EI) $\mathrm{m} / z$ calcd for $\mathrm{C}_{22} \mathrm{H}_{35} \mathrm{OSi}(\mathrm{M}-i$-Pr) 343.2457 , found 343.2445 . The $\mathbf{5 b \boldsymbol { \alpha }} / \mathbf{5} \mathbf{b} \boldsymbol{\beta}$ ratio was determined by GC/MS analysis of crude products: GC/MS $t_{\mathrm{R}}=23.1 \mathrm{~min}$ for $\mathbf{5 b \boldsymbol { \alpha } ,} 23.9 \mathrm{~min}$ for $\mathbf{5 b} \boldsymbol{\mathbf { b }}$. No detectable amount of $\mathbf{6 b}$ was obtained.<smiles>Cc1ccc2c(c1)C1(C)CCCC(O[I+])C1CC2</smiles>

Triisopropyl $\left(\left(1 S^{*}, 4 \mathrm{a} S^{*}, 10 \mathrm{a} R^{*}\right)\right.$-1,4a,6-trimethyl-1,2,3,4,4a,9,10,10a-octahydrophenanthren-1-yloxy)silane (5c $\beta$ ) (Entries 3-6, Table 1): Column chromatography (hexane); white solid; TLC, $R_{\mathrm{f}}=0.76$ (hexane-EtOAc $=4: 1$ ), 0.56 (hexane); IR (film) 3006, 2943, 2868, 1499, 1464, 1374, 1052, 993, $883 \mathrm{~cm}^{-1}$; ${ }^{\mathrm{H}} \mathrm{NMR}\left(\mathrm{CDCl}_{3}, 500 \mathrm{MHz}\right) \delta 1.00-1.16(\mathrm{~m}, 21 \mathrm{H}), 1.25-1.33(\mathrm{~m}, 1 \mathrm{H})$, $1.30(\mathrm{~s}, 3 \mathrm{H}), 1.32(\mathrm{~s}, 3 \mathrm{H}), 1.35(\mathrm{dt}, J=4.0,13.5 \mathrm{~Hz}, 1 \mathrm{H}), 1.43(\mathrm{dt}, J=3.5,13.0 \mathrm{~Hz}, 1 \mathrm{H}), 1.53-1.58(\mathrm{~m}$, $1 \mathrm{H}), 1.87$ (ddd, $J=3.0,4.5,13.5 \mathrm{~Hz}, 1 \mathrm{H}), 1.93-2.06(\mathrm{~m}, 3 \mathrm{H}), 2.28-2.31(\mathrm{~m}, 1 \mathrm{H}), 2.29(\mathrm{~s}, 3 \mathrm{H}), 2.79-$ $2.86(\mathrm{~m}, 1 \mathrm{H}), 2.94(\mathrm{ddd}, J=1.0,10.0,16.0 \mathrm{~Hz}, 1 \mathrm{H}), 6.89(\mathrm{dd}, J=1.0,7.5 \mathrm{~Hz}, 1 \mathrm{H}), 6.94(\mathrm{~d}, J=7.5 \mathrm{~Hz}$, 1H), $7.06(\mathrm{~s}, 1 \mathrm{H}) ;{ }^{13} \mathrm{C} \mathrm{NMR}\left(\mathrm{CDCl}_{3}, 125 \mathrm{MHz}\right) \delta 14.1(3 \mathrm{C}), 18.5$ (3C), $18.6(3 \mathrm{C}), 18.7,19.0,21.3,24.8$, 29.2, 30.4, 37.5, 38.6, 41.4, 50.9, 75.1, 124.6, 126.0, 128.8, 132.0, 134.7, 149.7; HRMS (EI) $\mathrm{m} / z$ calcd for $\mathrm{C}_{26} \mathrm{H}_{44} \mathrm{OSi}(\mathrm{M}) 400.3161$, found 400.3145 . The $\mathbf{5} \mathbf{c} / \mathbf{6}$ ratio was determined by ${ }^{1} \mathrm{H}$ NMR analysis of crude products: $6 \mathbf{c}$ was characterized by ${ }^{1} \mathrm{H}$ NMR chemical shifts $[\delta 4.59(\mathrm{~s}, 1 \mathrm{H}), 4.84(\mathrm{~s}, 1 \mathrm{H}), 5.43$ (brs, $1 \mathrm{H})$, other resonances could not be discerned.] and GC/MS analysis $(\mathrm{m} / \mathrm{z} 226[\mathrm{M}+1])$. The $\mathbf{5} \mathbf{c} \boldsymbol{\alpha} / \mathbf{5 c} \boldsymbol{\beta}$ ratio was determined by chiral HPLC analysis of crude products: HPLC (four linear OD-H columns, hexane $-i-\mathrm{PrOH}=2000 / 1$, flow rate $=0.3 \mathrm{~mL} / \mathrm{min}$ ) $t_{\mathrm{R}}=52.2 \mathrm{~min}$ for $\mathbf{5} \mathbf{c} \boldsymbol{\alpha}$ (inseparable enantiomers), 54.8 and $67.7 \mathrm{~min}$ for $\mathbf{5 b} \boldsymbol{\beta}$ enantiomers.

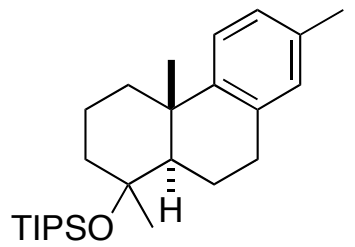

Triisopropyl $\left(\left(4 \mathrm{a} S^{*}, 10 \mathrm{a} R^{*}\right)-1,4 \mathrm{a}, 7\right.$-trimethyl-1,2,3,4,4a,9,10,10a-octahydrophenanthren-1-yloxy)silane (5d) (Entry 7, Table 1): Column chromatography (hexane); colorless oil; TLC, $R_{\mathrm{f}}=0.80$ (hexane-EtOAc $=4: 1$ ), 0.44 for $\alpha$ and 0.42 for $\beta$ (hexane); ${ }^{1} \mathrm{H}$ NMR $\left(\mathrm{CDCl}_{3}, 300 \mathrm{MHz}\right) \delta 1.00-2.34(\mathrm{~m}, 9 \mathrm{H}), 1.04-1.12(\mathrm{~m}, 21 \mathrm{H}), 1.15(\mathrm{~s}, 3 \mathrm{H}$ for $\alpha), 1.26(\mathrm{~s}, 3 \mathrm{H}$ for $\alpha), 1.30$ (s, $3 \mathrm{H}$ for $\beta$ ), 1.32 (s, $3 \mathrm{H}$ for $\beta$ ), $2.27(\mathrm{~s}, 3 \mathrm{H}), 2.77-3.00(\mathrm{~m}, 2 \mathrm{H}), 6.88-7.22(\mathrm{~m}, 3 \mathrm{H})$. The $\mathbf{5 d} / \mathbf{6 d}$ ratio was determined by ${ }^{1} \mathrm{H}$ NMR analysis of crude products: $\mathbf{6 d}$ was characterized by ${ }^{1} \mathrm{H}$ NMR chemical shifts [ $\delta 4.59(\mathrm{~s}, 1 \mathrm{H}), 4.85(\mathrm{~s}, 1 \mathrm{H}), 5.43$ (brs, $1 \mathrm{H})$, other resonances could not be discerned.] and GC/MS analysis $(\mathrm{m} / \mathrm{z}, 226[\mathrm{M}+1])$. The $\mathbf{5} \mathbf{d} \boldsymbol{\alpha} / \mathbf{5 d} \boldsymbol{\mathbf { \beta }}$ ratio was determined by chiral HPLC analysis of crude products: HPLC (two linear OD-H columns, hexane $-i-\mathrm{PrOH}=2000 / 1$, flow rate $=0.5 \mathrm{~mL} / \mathrm{min}$ ) $t_{\mathrm{R}}=$ 14.8 and $16.0 \mathrm{~min}$ for $\mathbf{5} \mathbf{d} \boldsymbol{\alpha}$ enantiomers, $17.1 \mathrm{~min}$ for $\mathbf{5} \mathbf{d} \boldsymbol{\beta}$ (inseparable enantiomers).

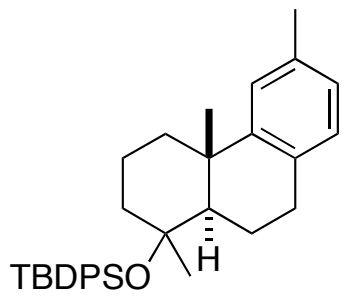

tert-Butyldiphenyl $\left(\left(4 \mathrm{a} S^{*}, 10 \mathrm{a} R^{*}\right)\right.$-1,4a,6-trimethyl-1,2,3,4,4a,9,10,10aoctahydrophenanthren-1-yloxy)silane (5e) (Entry 8, Table 1): Column chromatography (hexaneEtOAc $=50: 1$ ); amorphous solid; TLC, $R_{\mathrm{f}}=0.77$ (hexane-EtOAc $=4: 1$ ), 0.19 (hexane); ${ }^{1} \mathrm{H}$ NMR $\left(\mathrm{CDCl}_{3}, 500 \mathrm{MHz}\right) \delta 0.81-2.55(\mathrm{~m}, 11 \mathrm{H}), 1.02(\mathrm{~s}, 9 \mathrm{H}$ for $\alpha), 1.05$ (s, $9 \mathrm{H}$ for $\beta$ ), 1.08 (s, 3H for $\beta$ ), 1.10 (s, 3H for $\alpha$ ), 1.16 (s, 3H for $\alpha), 1.50$ (s, 3H for $\beta$ ), $2.30(\mathrm{~s}, 3 \mathrm{H}), 2.80-3.05(\mathrm{~m}, 2 \mathrm{H}), 6.90-7.09(\mathrm{~m}, 3 \mathrm{H})$, 7.30-7.44 (m, 6H), 7.66-7.75 (m, 4H for $\alpha), 7.79-7.88(\mathrm{~m}, 4 \mathrm{H}$ for $\beta)$. The $\mathbf{5 e} \boldsymbol{\alpha} / \mathbf{5 e} \boldsymbol{\beta} / \mathbf{6 e}(=\mathbf{6 c})$ ratio was 
determined by ${ }^{1} \mathrm{H}$ NMR analysis of crude products.

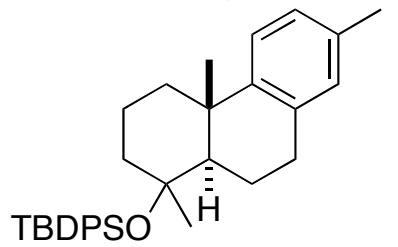

tert-Butyldiphenyl $((4 \mathrm{a} S *, 10 \mathrm{a} R *)-1,4 a, 7-t r i m e t h y l-1,2,3,4,4 \mathrm{a}, 9,10,10 \mathrm{a}-$ octahydrophenanthren-1-yloxy)silane (5f) (Entry 9, Table 1): Column chromatography (hexaneEtOAc $=50: 1$ ); amorphous solid; TLC, $R_{\mathrm{f}}=0.73$ (hexane-EtOAc $=4: 1$ ), 0.16 (hexane); ${ }^{1} \mathrm{H}$ NMR $\left(\mathrm{CDCl}_{3}, 500 \mathrm{MHz}\right) \delta 1.00-2.49(\mathrm{~m}, 9 \mathrm{H}), 1.02(\mathrm{~s}, 9 \mathrm{H}$ for $\alpha), 1.05(\mathrm{~s}, 9 \mathrm{H}$ for $\beta), 1.07$ (s, 3H for $\left.\beta\right), 1.09$ (s, $3 \mathrm{H}$ for $\alpha$ ), $1.16(\mathrm{~s}, 3 \mathrm{H}$ for $\alpha$ ), 1.49 (s, 3H for $\beta$ ), 2.28 (s, 3H for $\beta$ ), 2.29 (s, 3H for $\alpha$ ), 2.80-3.12 (m, 2H), 6.89-6.97 (m, 2H), 7.12-7.19 (m, 1H), 7.31-7.44 (m, 6H), 7.66-7.76 (m, 4H for $\alpha), 7.79-7.88(\mathrm{~m}$, $4 \mathrm{H}$ for $\beta)$. The $\mathbf{5 f} \boldsymbol{\alpha} / \mathbf{5 f} \beta / \mathbf{6 f}(=\mathbf{6 d})$ ratio was determined by ${ }^{1} \mathrm{H} \mathrm{NMR}$ analysis of crude products.

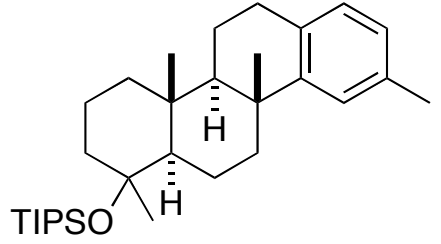

Triisopropyl $\left(\left(4 \mathrm{a} R^{*}, 4 \mathrm{~b} R^{*}, 10 \mathrm{~b} R^{*}, 12 \mathrm{a} R^{*}\right)-1,4 \mathrm{a}, 9,10 \mathrm{~b}\right.$-tetramethyl1,2,3,4,4a,4b,5,6,10b,11,12,12a-dodecahydrochrysen-1-yloxy)silane (8a) (entry 1, Table 2): Column chromatography (hexane); colorless oil (as an inseparable mixture of 8a and 9a); TLC, $R_{\mathrm{f}}=0.81$ (hexane-EtOAc = 4:1), 0.37 (hexane); ${ }^{1} \mathrm{H}$ NMR $\left(\mathrm{CDCl}_{3}, 300 \mathrm{MHz}\right) \delta 0.80-3.00(\mathrm{~m}, 16 \mathrm{H}), 0.88(\mathrm{~s}, 3 \mathrm{H}$ for $\alpha), 1.02-1.12(\mathrm{~m}, 21 \mathrm{H}), 1.07$ (s, 3H for $\beta$ ), $1.18(\mathrm{~s}, 3 \mathrm{H}$ for $\alpha), 1.20$ (s, 3H for $\alpha), 1.24$ (s, 3H for $\beta$ ), $1.27(\mathrm{~s}, 3 \mathrm{H}$ for $\beta), 2.30(\mathrm{~s}, 3 \mathrm{H}), 6.89-7.11(\mathrm{~m}, 3 \mathrm{H})$. The $\mathbf{8 a} / \mathbf{9 a}$ ratio was determined by ${ }^{1} \mathrm{H}$ NMR analysis of crude products: 9a was characterized by ${ }^{1} \mathrm{H}$ NMR chemical shifts $[\delta 4.53$ (s, 1H), 4.73 (s, $1 \mathrm{H}), 5.30$ (1brs, $1 \mathrm{H})$, other resonances could not be discerned.]. The $\mathbf{8 a} \boldsymbol{\alpha} / \mathbf{8 a} \boldsymbol{\beta}$ ratio was determined by the separation of the corresponding alcohols after desilylation with TBAF.

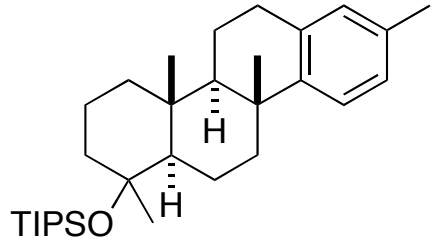

Triisopropyl $((4 \mathrm{a} R *, 4 \mathrm{~b} R *, 10 \mathrm{~b} R *, 12 \mathrm{a} R *)-1,4 \mathrm{a}, 8,10 \mathrm{~b}-$ tetramethyl1,2,3,4,4a,4b,5,6,10b,11,12,12a-dodecahydrochrysen-1-yloxy)silane (8b) (Entry 2, Table 2): Column chromatography (hexane); colorless oil (as an inseparable mixture of $\mathbf{8 b}$ and $\mathbf{9 b}$ ); TLC, $R_{\mathrm{f}}=$ 0.85 (hexane-EtOAc $=4: 1), R_{\mathrm{f}}=0.50$ (hexane); ${ }^{1} \mathrm{H}$ NMR $\left(\mathrm{CDCl}_{3}, 300 \mathrm{MHz}\right) \delta 0.82-2.44(\mathrm{~m}, 14 \mathrm{H})$, $0.88(\mathrm{~s}, 3 \mathrm{H}$ for $\alpha), 1.01-1.11(\mathrm{~m}, 21 \mathrm{H}), 1.07$ (s, 3H for $\beta$ ), $1.18(\mathrm{~s}, 3 \mathrm{H}$ for $\alpha), 1.19$ (s, 3H for $\alpha), 1.23$ (s, $3 \mathrm{H}$ for $\beta), 1.26(\mathrm{~s}, 3 \mathrm{H}$ for $\beta), 2.27(\mathrm{~s}, 3 \mathrm{H}), 2.74-2.93(\mathrm{~m}, 2 \mathrm{H}), 6.86-7.19(\mathrm{~m}, 3 \mathrm{H})$. The 8b/9b ratio was determined by ${ }^{1} \mathrm{H}$ NMR analysis of crude products: 9b was characterized by ${ }^{1} \mathrm{H}$ NMR chemical shifts [ $\delta 4.53(\mathrm{~s}, 1 \mathrm{H}), 4.74(\mathrm{~s}, 1 \mathrm{H}), 5.31$ (1brs, 1H), other resonances could not be discerned.]. The $\mathbf{8 b \boldsymbol { \alpha }} / \mathbf{8 b} \boldsymbol{\beta}$ ratio was determined by chiral HPLC analysis of crude products: HPLC (two linear OD-H columns, hexane $-i-\mathrm{PrOH}=2000: 1$, flow rate $=0.3 \mathrm{~mL} / \mathrm{min}$ ) $t_{\mathrm{R}}=26.2$ and $30.5 \mathrm{~min}$ for $\mathbf{8 b} \alpha$ enantiomers, 28.5 and $38.2 \mathrm{~min}$ for $\mathbf{8 b} \beta$ enantiomers.

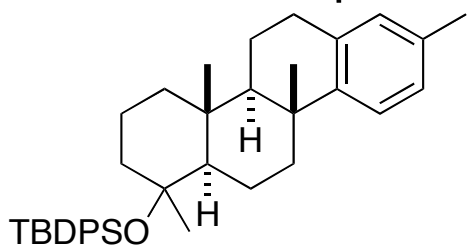

tert-Butyldiphenyl $\left(\left(4 \mathrm{a} R^{*}, 4 \mathrm{~b} R^{*}, 10 \mathrm{~b} R^{*}, 12 \mathrm{a} R^{*}\right)-1,4 \mathrm{a}, 8,10 \mathrm{~b}\right.$-tetramethyl1,2,3,4,4a,4b,5,6,10b,11,12,12a-dodecahydrochrysen-1-yloxy)silane (8c) (Entry 3, Table 2): Column chromatography (hexane-EtOAc $=50: 1$ ); amorphous solid; TLC, $R_{\mathrm{f}}=0.65$ (hexane-EtOAc $=$ 4:1), 0.18 (hexane); ${ }^{1} \mathrm{H} \mathrm{NMR}\left(\mathrm{CDCl}_{3}, 300 \mathrm{MHz}\right) \delta 0.77-2.50(\mathrm{~m}, 14 \mathrm{H}), 0.84(\mathrm{~s}, 3 \mathrm{H}$ for $\alpha), 0.90(\mathrm{~s}, 3 \mathrm{H}$ for $\beta$ ), 1.01 (s, 9H for $\alpha$ ), 1.03 (s, 3H for $\beta$ ), 1.06 (s, 9H for $\beta$ ), 1.12 (s, 3H for $\alpha$ ), 1.22 (s, 3H for $\alpha$ ), 
$1.45(\mathrm{~s}, 3 \mathrm{H}$ for $\beta), 2.30(\mathrm{~s}, 3 \mathrm{H}), 2.75-2.99(\mathrm{~m}, 2 \mathrm{H}), 6.89(\mathrm{~s}, 1 \mathrm{H}), 6.98(\mathrm{~d}, J=7.8 \mathrm{~Hz}, 1 \mathrm{H}), 7.24(\mathrm{~d}, J=$ $7.8 \mathrm{~Hz}, 1 \mathrm{H}), 7.30-7.44(\mathrm{~m}, 6 \mathrm{H}), 7.30-7.44(\mathrm{~m}, 6 \mathrm{H}), 7.64-7.74(\mathrm{~m}, 4 \mathrm{H}$ for $\alpha), 7.80-7.86(\mathrm{~m}, 4 \mathrm{H}$ for $\beta$ ). The $\mathbf{8 c} \boldsymbol{\alpha} / \mathbf{8 c} \beta$ ratio was determined by ${ }^{1} \mathrm{H}$ NMR analysis of crude products. No detectable amount of $9 \mathbf{c}$ was obtained.<smiles>Cc1ccc2c(c1)C1(C)CCC[C@](C)(OS(C)(F)F)[C@]1(C)CC2</smiles>

Triisopropyl $\left(\left(1 S^{*}, 4 \mathrm{a} S^{*}, 10 \mathrm{a} S^{*}\right)-1,4 \mathrm{a}, 6\right.$-trimethyl-1,2,3,4,4a,9,10,10a-octahydrophenanthren-1-yloxy)silane (11a $\beta$ ) (Entry 1, Table 3): Column chromatography (hexane); colorless oil; TLC, $R_{\mathrm{f}}=0.77$ (hexane-EtOAc = 4:1), 0.35 (hexane); IR (film) 2944, 2866, 2360, 1502, 1464, 1378, 1130, 1054, 1013, $883 \mathrm{~cm}^{-1} ;{ }^{1} \mathrm{H} \mathrm{NMR}\left(\mathrm{CDCl}_{3}, 500 \mathrm{MHz}\right) \delta 0.86(\mathrm{~s}, 3 \mathrm{H}), 1.08(\mathrm{~m}, 21 \mathrm{H})$, $1.33(\mathrm{~s}, 3 \mathrm{H}), 1.33-1.41(\mathrm{~m}, 1 \mathrm{H}), 1.46-1.51(\mathrm{~m}, 1 \mathrm{H}), 1.62(\mathrm{t}, J=6.0 \mathrm{~Hz}, 2 \mathrm{H}), 1.66-1.75(\mathrm{~m}, 1 \mathrm{H}), 1.80(\mathrm{t}$, $J=5.5 \mathrm{~Hz}, 1 \mathrm{H}), 2.00-2.13(\mathrm{~m}, 2 \mathrm{H}), 2.16-2.24(\mathrm{~m}, 1 \mathrm{H}), 2.32(\mathrm{~s}, 3 \mathrm{H}), 2.76-2.90(\mathrm{~m}, 2 \mathrm{H}), 6.90(\mathrm{~d}, J=7.5$ $\mathrm{Hz}, 1 \mathrm{H}), 6.93(\mathrm{~d}, J=7.5 \mathrm{~Hz}, 1 \mathrm{H}), 7.06(\mathrm{~s}, 1 \mathrm{H}) ;{ }^{13} \mathrm{C} \mathrm{NMR}\left(\mathrm{CDCl}_{3}, 125 \mathrm{MHz}\right) \delta 13.8(3 \mathrm{C}), 18.5(3 \mathrm{C})$, 18.6 (3C), 18.8, 20.2, 21.4, 26.3, 27.1, 32.4, 37.1, 38.5, 41.9, 52.4, 76.5, 125.2, 125.9 (2C), 128.6, 133.8, 134.7; HRMS (EI) $\mathrm{m} / \mathrm{z}$ calcd for $\mathrm{C}_{26} \mathrm{H}_{44} \mathrm{OSi}(\mathrm{M})$ 400.3161, found 400.3165. No detectable amounts of $11 \mathrm{a} \alpha$ and $12 \mathrm{a}$ were obtained.<smiles>Cc1ccc2c(c1)CCC1C(O)(OC(F)(F)F)CCCC21C</smiles>

Triisopropyl((4aS*,10a $\left.S^{*}\right)$-1,4a,7-trimethyl-1,2,3,4,4a,9,10,10a-octahydrophenanthren-1-yloxy)silane (11b) (Entry 2, Table 3): Column chromatography (hexane); colorless oil; TLC, $R_{\mathrm{f}}=0.72$ (hexane-EtOAc $\left.=4: 1\right), 0.49$ (hexane); ${ }^{1} \mathrm{H} \mathrm{NMR}\left(\mathrm{CDCl}_{3}, 300 \mathrm{MHz}\right) \delta 0.86(\mathrm{~s}, 3 \mathrm{H}$ for $\beta$ ), 0.96 (s, 3H for $\alpha$ ), 0.98 (s, 3H for $\alpha$ ), 1.06 (brs, 21H), 1.25-2.17 (m, 9H), 1.32 (s, 3H for $\beta$ ), 2.27 (s, $3 \mathrm{H}), 2.72-2.92(\mathrm{~m}, 2 \mathrm{H}), 6.82(\mathrm{~s}, 1 \mathrm{H}$ for $\alpha), 6.85(\mathrm{~s}, 1 \mathrm{H}$ for $\beta), 6.90-6.93(\mathrm{~m}, 1 \mathrm{H}$ for $\alpha), 6.93(\mathrm{~d}, J=8.1$ $\mathrm{Hz}, 1 \mathrm{H}$ for $\beta), 7.13(\mathrm{~d}, J=8.1 \mathrm{~Hz}, 1 \mathrm{H}$ for $\beta), 7.17(\mathrm{~d}, J=8.1 \mathrm{~Hz}, 1 \mathrm{H}$ for $\alpha)$. The $\mathbf{1 1 b} / \mathbf{1 2 b}$ ratio was determined by ${ }^{1} \mathrm{H}$ NMR analysis of crude products. 12b was characterized by ${ }^{1} \mathrm{H}$ NMR chemical shifts [ $\delta 4.74(\mathrm{~s}, 2 \mathrm{H}), 5.42$ (brs, $1 \mathrm{H})$, other resonances could not be discerned.] and GC/MS analysis $(\mathrm{m} / z 226$ $[\mathrm{M}+1])$. The $\mathbf{1 1 b} \boldsymbol{\alpha} / \mathbf{1 1 b} \boldsymbol{\beta}$ ratio was determined by GC/MS analysis of crude products: GC/MS $t_{\mathrm{R}}=23.4$ $\min$ for $\mathbf{5 b} \boldsymbol{b}, 23.5 \mathrm{~min}$ for $\mathbf{5 b} \boldsymbol{b}$.

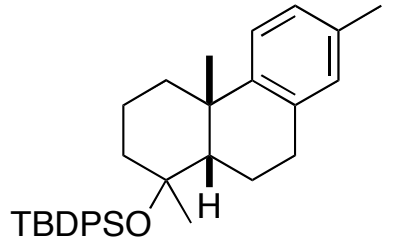

tert-Butyldiphenyl $((4 \mathrm{a} S *, 10 \mathrm{a} S *)-1,4 a, 7-t r i m e t h y l-1,2,3,4,4 a, 9,10,10 a-$ octahydrophenanthren-1-yloxy)silane (11c) (Entry 3, Table 3): Column chromatography (hexaneEtOAc $=50: 1)$; colorless oil; TLC, $R_{\mathrm{f}}=0.74$ (hexane-EtOAc $\left.=4: 1\right), 0.14$ (hexane); ${ }^{1} \mathrm{H}$ NMR $\left(\mathrm{CDCl}_{3}\right.$, $300 \mathrm{MHz}) \delta 0.67(\mathrm{~s}, 3 \mathrm{H}$ for $\beta$ ), $0.80(\mathrm{~s}, 9 \mathrm{H}$ for $\alpha), 1.02$ (s, 9H for $\beta$ ), 1.02-2.34 (m, $8 \mathrm{H}$ for $\alpha$ and $9 \mathrm{H}$ for $\beta$ ), 1.24 (s, 3H for $\alpha$ ), 1.27 (s, 3H for $\beta$ ), 1.28 (s, 3H for $\alpha$ ), 2.25 (s, 3H for $\beta$ ), 2.27 (s, 3H for $\alpha$ ), 2.42$2.53(\mathrm{~m}, 1 \mathrm{H}$ for $\alpha), 2.70-2.91 \mathrm{~m}, 1 \mathrm{H}$ for $\alpha$ and $2 \mathrm{H}$ for $\beta$ ), 3.10-3.22 (m, $1 \mathrm{H}$ for $\alpha), 6.83-7.07(\mathrm{~m}, 3 \mathrm{H})$, 7.21-7.43 (m, 6H), 7.68-7.77 $(\mathrm{m}, 4 \mathrm{H})$. The 11c $\alpha / \mathbf{1 1 c} \beta$ ratio was determined by ${ }^{1} \mathrm{H} \mathrm{NMR}$ analysis of crude products. No detectable amount of $12 \mathrm{c}$ was obtained. 
<smiles>Cc1ccc2c(c1)C1(C)CCCC(OS)C1CC2</smiles>

$((1 R *, 4 \mathrm{a} S *, 10 \mathrm{a} R *)-4 \mathrm{a}, 6-D i m e t h y l-1,2,3,4,4 \mathrm{a}, 9,10,10 \mathrm{a}-0 \mathrm{ctahydrophenanthren-1-}$ yloxy)triisopropylsilane $(\mathbf{1 6 a} \alpha)$ (Entry 3, Table 4): Column chromatography (hexane-EtOAc = 20:1); colorless oil; TLC, $R_{\mathrm{f}}=0.66$ (hexane-EtOAc $=4: 1$ ), 0.13 (hexane); IR (film) 2943, 2867, 1464, 1380, 1114, 1091, 1067, $882 \mathrm{~cm}^{-1}$; ${ }^{1} \mathrm{H}$ NMR $\left(\mathrm{CDCl}_{3}, 500 \mathrm{MHz}\right) \delta 1.00-1.77(\mathrm{~m}, 6 \mathrm{H}), 1.03-1.09(\mathrm{~m}$, 24H), $1.32(\mathrm{~s}, 3 \mathrm{H}), 2.03-2.35(\mathrm{~m}, 3 \mathrm{H}), 2.29(\mathrm{~s}, 3 \mathrm{H}), 2.74-2.87(\mathrm{~m}, 2 \mathrm{H}), 3.76(\mathrm{td}, J=4.2,9.9 \mathrm{~Hz}, 1 \mathrm{H})$, $6.90(\mathrm{~d}, J=7.8 \mathrm{~Hz}, 1 \mathrm{H}), 6.96(\mathrm{~d}, J=7.8 \mathrm{~Hz}, 1 \mathrm{H}), 7.06(\mathrm{~s}, 1 \mathrm{H})$; HRMS (EI) $\mathrm{m} / z$ calcd for $\mathrm{C}_{25} \mathrm{H}_{42} \mathrm{OSi}(\mathrm{M})$ 386.3005, found 386.2991. The 16a $\alpha / \mathbf{1 6 a} \beta$ ratio was determined by ${ }^{1} \mathrm{H}$ NMR analysis of crude products.<smiles>Cc1ccc2c(c1)C1(C)CCCC(OC(F)(F)F)C1CC2</smiles>

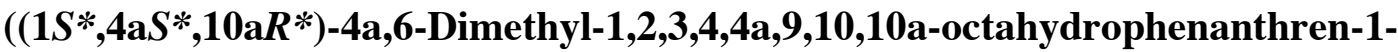
yloxy)triisopropylsilane (16a $\beta$ ) (Entry 3, Table 4): Column chromatography (hexane); white solid; TLC, $R_{\mathrm{f}}=0.76$ (hexane-EtOAc $=4: 1$ ), $R_{\mathrm{f}}=0.56$ (hexane); IR (film) 2943, 2867, 1464, 1132, 1084, 1033, 932, $883 \mathrm{~cm}^{-1} ;{ }^{1} \mathrm{H}$ NMR $\left(\mathrm{CDCl}_{3}, 500 \mathrm{MHz}\right) \delta 0.90-1.14(\mathrm{~m}, 21 \mathrm{H}), 1.32(\mathrm{~s}, 3 \mathrm{H}), 1.38-1.51(\mathrm{~m}$, $3 \mathrm{H}), 1.55-1.64(\mathrm{~m}, 2 \mathrm{H}), 1.86(\mathrm{dd}, J=2.0,13.5 \mathrm{~Hz}, 1 \mathrm{H}), 2.03(\mathrm{tq}, J=3.5,13.5 \mathrm{~Hz}, 1 \mathrm{H}), 2.16(\mathrm{ddt}, J=$ 8.0, 10.8, $13.0 \mathrm{~Hz}, 1 \mathrm{H}), 2.25-2.33(\mathrm{~m}, 1 \mathrm{H}), 2.29(\mathrm{~s}, 3 \mathrm{H}), 2.82-2.92(\mathrm{~m}, 2 \mathrm{H}), 4.16(\mathrm{dd}, J=2.0,5.0 \mathrm{~Hz}$, $1 \mathrm{H}), 6.89(\mathrm{dd}, J=1.5,7.5 \mathrm{~Hz}, 1 \mathrm{H}), 6.94(\mathrm{~d}, J=7.5 \mathrm{~Hz}, 1 \mathrm{H}), 7.05(\mathrm{~s}, 1 \mathrm{H}) ;{ }^{13} \mathrm{C} \mathrm{NMR}\left(\mathrm{CDCl}_{3}, 125 \mathrm{MHz}\right)$ ठ 12.8 (3C), 17.7, 18.3 (6C), 21.3, 23.9, 25.7, 29.3, 34.5, 37.0, 38.2, 46.0, 73.0, 124.8, 126.1, 129.0, 132.0, 134.8, 149.2; HRMS (EI) $m / z$ calcd for $\mathrm{C}_{25} \mathrm{H}_{42} \mathrm{OSi}(\mathrm{M}) 386.3005$, found 386.2991. The $\mathbf{1 6} \mathbf{a} \alpha / \mathbf{1 6} \mathbf{\beta}$ ratio was determined by ${ }^{1} \mathrm{H}$ NMR analysis of crude products.<smiles>Cc1ccc2c(c1)CC[C@@H]1[C@H](OS(C)(F)F)CCC[C@@]21C</smiles>

$\left(\left(1 R^{*}, 4 \mathrm{a} S *, 10 \mathrm{a} R *\right)-4 \mathrm{a}, 7-D i m e t h y l-1,2,3,4,4 \mathrm{a}, 9,10,10 \mathrm{a}-0 \mathrm{ctahydrophenanthren-1-}\right.$ yloxy)triisopropylsilane (16ba) (Entries 4 and 5, Table 4): A small amount of 5-Me-regioisomer was contained; column chromatography (hexane-EtOAc $=20: 1$ ); colorless oil; TLC, $R_{\mathrm{f}}=0.80$ (hexaneEtOAc = 4:1), 0.12 (hexane); ${ }^{1} \mathrm{H}$ NMR $\left(\mathrm{CDCl}_{3}, 300 \mathrm{MHz}\right) \delta 0.97-1.78(\mathrm{~m}, 6 \mathrm{H}), 0.97-1.09(\mathrm{~m}, 24 \mathrm{H})$, 1.21 (s, 3H for 5-Me-regioisomer), 2.03-2.50 (m, 3H), $2.28(\mathrm{~s}, 3 \mathrm{H}$ for 7-Me-regioisomer), $2.50(\mathrm{~s}, 3 \mathrm{H}$ for 5-Me-regioisomer), 2.80-2.87 (m, 2H), $3.76(\mathrm{td}, J=4.5,9.6 \mathrm{~Hz}, 1 \mathrm{H}), 6.89(\mathrm{~s}, 1 \mathrm{H}), 6.94(\mathrm{dm}, J=7.8$ $\mathrm{Hz}, 1 \mathrm{H}), 7.15(\mathrm{~d}, J=7.8 \mathrm{~Hz}, 1 \mathrm{H})$. The $\mathbf{1 6 b} \boldsymbol{\alpha} / \mathbf{1 6 b} \beta$ ratio was determined by ${ }^{1} \mathrm{H} \mathrm{NMR}$ analysis of crude products.<smiles>Cc1ccc2c(c1)CC[C@@H]1[C@H](OS(C)(F)F)CCC[C@@]21C</smiles>

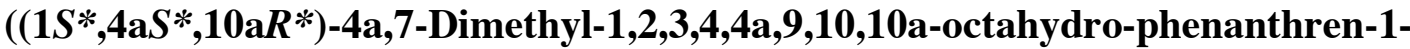
yloxy)triisopropylsilane (16b $\beta$ ) (Entries 4 and 5, Table 4): Column chromatography (hexane); colorless oil; TLC, $R_{\mathrm{f}}=0.42$ (hexane); IR (film) 3006, 2941, 2867, 1496, 1464, 1383, 1084, 1033, 931, $884 \mathrm{~cm}^{-1} ;{ }^{1} \mathrm{H} \mathrm{NMR}\left(\mathrm{CDCl}_{3}, 500 \mathrm{MHz}\right) \delta 1.10(\mathrm{~m}, 21 \mathrm{H}), 1.31(\mathrm{~s}, 3 \mathrm{H}), 1.31-1.66(\mathrm{~m}, 5 \mathrm{H}), 1.87$ (brd, $J=$ 
$13.2 \mathrm{~Hz}, 1 \mathrm{H}), 2.02(\mathrm{tq}, J=3.6,13.5 \mathrm{~Hz}, 1 \mathrm{H}), 2.12-2.30(\mathrm{~m}, 1 \mathrm{H}), 2.27(\mathrm{~s}, 3 \mathrm{H}), 2.84-2.92(\mathrm{~m}, 2 \mathrm{H}), 4.16$ $(\mathrm{d}, J=2.4 \mathrm{~Hz}, 1 \mathrm{H}), 6.87(\mathrm{~s}, 1 \mathrm{H}), 6.94(\mathrm{~d}, J=8.1 \mathrm{~Hz}, 1 \mathrm{H}), 7.14(\mathrm{~d}, J=8.1 \mathrm{~Hz}, 1 \mathrm{H}) ;{ }^{13} \mathrm{C} \mathrm{NMR}\left(\mathrm{CDCl}_{3}\right.$, $125 \mathrm{MHz}) \delta 12.8$ (3C), 17.8, 18.3 (6C), 20.8, 23.8, 25.8, 29.6, 34.5, 36.7, 38.3, 46.1, 72.9, 124.2, 126.4, 129.7, 134.6, 135.1, 146.5; HRMS (EI) $\mathrm{m} / z$ calcd for $\mathrm{C}_{25} \mathrm{H}_{42} \mathrm{OSi}$ (M) 386.3005, found 386.2980. The $\mathbf{1 6 b} \boldsymbol{\alpha} / \mathbf{1 6 b} \beta$ ratio was determined by ${ }^{1} \mathrm{H}$ NMR analysis of crude products.

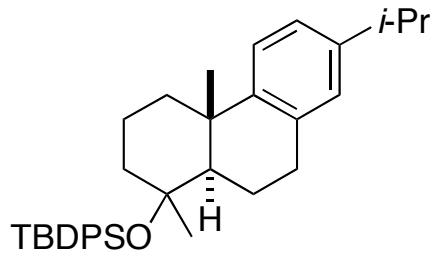

tert-Butyl $\left(\left(1 R^{*}, 4 \mathrm{a} S *, 10 \mathrm{a} R^{*}\right)-7\right.$-isopropyl-1,4a-dimethyl1,2,3,4,4a,9,10,10a-octahydrophenanthren-1-yloxy)diphenylsilane (5h) (Scheme 2): Column chromatography (hexane-EtOAc $=50: 1 \rightarrow 20: 1$ ); amorphous solid; TLC (hexane-EtOAc $=25: 1) R_{\mathrm{f}}=$ 0.35; IR (film) 3004, 2961, 2859, 1471, 1427, 1383, 1107, 1049, 909, $822 \mathrm{~cm}^{-1} ;{ }^{1} \mathrm{H}$ NMR $\left(\mathrm{CDCl}_{3}, 500\right.$ $\mathrm{MHz}) \delta 1.03(\mathrm{~s}, 9 \mathrm{H}), 1.05(\mathrm{~s}, 3 \mathrm{H}), 1.16(\mathrm{~s}, 3 \mathrm{H}), 1.24(\mathrm{~d}, J=7.0 \mathrm{~Hz}, 6 \mathrm{H}), 1.33-1.56(\mathrm{~m}, 5 \mathrm{H}), 1.67-1.76$ $(\mathrm{m}, 1 \mathrm{H}), 1.96(\mathrm{dd}, J=2.0,12.5 \mathrm{~Hz}, 1 \mathrm{H}), 2.10-2.16(\mathrm{~m}, 1 \mathrm{H}), 2.40-2.44(\mathrm{~m}, 1 \mathrm{H}), 2.80-3.00(\mathrm{~m}, 3 \mathrm{H}), 6.92$ (brs, $1 \mathrm{H}$ for $\beta), 6.94(\mathrm{~d}, J=1.5 \mathrm{~Hz}, 1 \mathrm{H}$ for $\alpha), 7.00(\mathrm{dd}, J=2.0,8.0 \mathrm{~Hz}, 1 \mathrm{H}), 7.15(\mathrm{~d}, J=8.0 \mathrm{~Hz}, 1 \mathrm{H}$ for $\alpha), 7.19(\mathrm{~d}, J=8.5 \mathrm{~Hz}, 1 \mathrm{H}$ for $\beta$ ), 7.30-7.43 (m, 6H), 7.66-7.74 (m, 4H for $\alpha$ ), 7.80-7.86 (m, 4H for $\beta$ ); ${ }^{13} \mathrm{C} \mathrm{NMR}\left(\mathrm{CDCl}_{3}, 125 \mathrm{MHz}\right) \delta 18.4,19.5,20.6,24.0$ (3C), 24.6, 27.2, 30.6, 33.4, 37.8, 38.2, 42.1, 52.9, 77.6, 123.9, 124.6, 127.0 (2C), 127.3 (2C), 129.2 (2C), 129.3, 135.1, 136.1 (2C), 136.3 (4C), 145.6, 146.9; HRMS (EI) $m / z$ calcd for $\mathrm{C}_{31} \mathrm{H}_{37} \mathrm{OSi}(\mathrm{M}-t-\mathrm{Bu}) 453.2614$, found 453.2952. The $5 \mathrm{~h} \alpha / 5 \mathrm{~h} \beta$ ratio was determined by ${ }^{1} \mathrm{H}$ NMR analysis of crude products.<smiles>CCCc1ccc2c(c1)CCC1(C(F)(F)F)C(O)CCCC21C</smiles>

Triisopropyl((1S*,4a $\left.S^{*}, 10 \mathrm{a} R *\right)-7$-isopropyl-4a-methyl-1,2,3,4,4a,9,10,10aoctahydrophenanthren-1-yloxy)silane (16c $\beta$ ) (Scheme 2): Column chromatography (hexane); colorless oil; TLC, $R_{\mathrm{f}}=0.41$ (hexane); IR (film) 2930, 2867, 1457, 1085, 1033, $883 \mathrm{~cm}^{-1}$; ${ }^{1} \mathrm{H}$ NMR $\left(\mathrm{CDCl}_{3}, 500 \mathrm{MHz}\right) \delta 1.10(\mathrm{~m}, 21 \mathrm{H}), 1.22(\mathrm{~d}, J=7.0 \mathrm{~Hz}, 6 \mathrm{H}), 1.32(\mathrm{~s}, 3 \mathrm{H}), 1.38-1.65(\mathrm{~m}, 5 \mathrm{H}), 1.86(\mathrm{dm}$, $J=13.5 \mathrm{~Hz}, 1 \mathrm{H}), 2.03(\mathrm{tq}, J=3.5,13.5 \mathrm{~Hz}, 1 \mathrm{H}), 2.13-2.22(\mathrm{~m}, 1 \mathrm{H}), 2.17(\mathrm{~s}, 3 \mathrm{H}), 2.27(\mathrm{brd}, J=13.0 \mathrm{~Hz}$, $1 \mathrm{H}), 2.78-2.86(\mathrm{~m}, 1 \mathrm{H}), 2.88-2.92(\mathrm{~m}, 2 \mathrm{H}), 4.17(\mathrm{dm}, J=2.5 \mathrm{~Hz}, 1 \mathrm{H}), 6.89(\mathrm{~d}, J=1.0 \mathrm{~Hz}, 1 \mathrm{H}), 6.98$ $(\mathrm{dd}, J=1.5,8.0 \mathrm{~Hz}, 1 \mathrm{H}), 7.16(\mathrm{~d}, J=8.0 \mathrm{~Hz}, 1 \mathrm{H}) ;{ }^{13} \mathrm{C} \mathrm{NMR}\left(\mathrm{CDCl}_{3}, 125 \mathrm{MHz}\right) \delta 12.8(3 \mathrm{C}), 17.8,18.3$ (6C), 23.9, 24.0, 25.8, 29.8, 30.9, 33.5, 34.5, 36.8, 38.2, 46.1, 73.0, 123.7, 124.1, 127.0, 134.9, 145.4, 146.8; HRMS (EI) $\mathrm{m} / z$ calcd for $\mathrm{C}_{27} \mathrm{H}_{46} \mathrm{OSi}(\mathrm{M}) 414.3318$, found 414.3312. No detectable amounts of $16 \mathbf{c} \beta$ and 18c were obtained.

General Procedure for the Desilylation of 4-Siloxy Polycycles: To a round-bottomed flask charged with 4-siloxy polycycle $(\mathbf{5}, \mathbf{8}, \mathbf{1 1}, \mathbf{1 6})$ was added tetrabutylammonium fluoride (a 1.0 M THF solution, TCI (Tokyo Chemical Industry Co., Ltd., Japan), 2-10 equiv), and the mixture was refluxed for 2-12 h. The reaction was quenched with water, and the aqueous layer was extracted with EtOAc (3 times). The combined organic layers were dried over anhydrous $\mathrm{MgSO}_{4}$ and concentrated in vacuo. The crude product was purified by column chromatography on silica gel to give the corresponding alcohol almost in quantitative yield. 
<smiles>CC12CCC[C@@H](O)[C@H]1CCc1ccc(F)cc12</smiles>

$\left(1 S^{*}, 4 \mathrm{a} S^{*}, 10 \mathrm{a} R^{*}\right)-6-F l u o r o-1,4 a-d i m e t h y l-1,2,3,4,4 a, 9,10,10 a-o c t a h y d r o-$

phenanthren-1-ol (5a $\beta-O H)$ (Table 1): Column chromatography (hexane-EtOAc = 5:1); colorless oil; TLC, $R_{\mathrm{f}}=0.29$ (hexane-EtOAc = 4:1); IR (film) 3608, 2936, 1587, 1496, 1375, 941, $868 \mathrm{~cm}^{-1}$; ${ }^{1} \mathrm{H}$ NMR $\left(\mathrm{CDCl}_{3}, 500 \mathrm{MHz}\right) \delta 1.03(\mathrm{~s}, \mathrm{OH}), 1.26(\mathrm{~s}, 3 \mathrm{H}), 1.30(\mathrm{~s}, 3 \mathrm{H}), 1.36-1.45(\mathrm{~m}, 3 \mathrm{H}), 1.60-1.66(\mathrm{~m}, 1 \mathrm{H})$, $1.74(\mathrm{~d}, J=14.0 \mathrm{~Hz}, 1 \mathrm{H}), 1.87(\mathrm{ddt}, J=7.5,10.5,13.0 \mathrm{~Hz}, 1 \mathrm{H}), 1.95(\mathrm{ttd}, J=3.5,7.0,14.0 \mathrm{~Hz}, 1 \mathrm{H})$, $2.00-2.05(\mathrm{~m}, 1 \mathrm{H}), 2.23(\mathrm{~d}, J=12.5 \mathrm{~Hz}, 1 \mathrm{H}), 2.82-2.89(\mathrm{~m}, 1 \mathrm{H}), 2.96(\mathrm{dd}, J=7.5,17.0 \mathrm{~Hz}, 1 \mathrm{H}), 6.78$ $(\mathrm{dt}, J=2.5,8.5 \mathrm{~Hz}, 1 \mathrm{H}), 6.94(\mathrm{dd}, J=2.5,11.0 \mathrm{~Hz}, 1 \mathrm{H}), 6.99(\mathrm{dd}, J=6.0,8.5 \mathrm{~Hz}, 1 \mathrm{H}) ;{ }^{13} \mathrm{C}$ NMR $\left(\mathrm{CDCl}_{3}, 125 \mathrm{MHz}\right) \delta 17.8,18.3,24.2,28.5,0.8,37.7,38.1,40.7,48.3,72.1,110.6,112.2,130.1,130.4$, $151.4,161.1\left(\mathrm{~d}, J_{\mathrm{CF}}=240 \mathrm{~Hz}\right) ;{ }^{19} \mathrm{~F} \mathrm{NMR}\left(\mathrm{CDCl}_{3}, 282 \mathrm{MHz}\right) \delta-118.2(\mathrm{dd}, J=6.6,18.2 \mathrm{~Hz})$; HRMS (EI) $m / z$ calcd for $\mathrm{C}_{17} \mathrm{H}_{21} \mathrm{FO}(\mathrm{M}) 248.1576$, found 248.1552 .

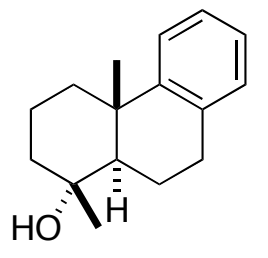

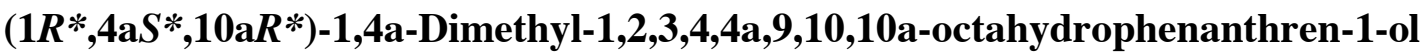
(5bo-OH) (Table 1): Column chromatography (hexane-EtOAc = 5:1); colorless oil; TLC (hexaneEtOAc $=4: 1) R_{\mathrm{f}}=0.22$; IR (film) 3595, 3010, 2930, 2863, 2360, 2320, 1457, $1381 \mathrm{~cm}^{-1} ;{ }^{1} \mathrm{H}$ NMR $\left(\mathrm{CDCl}_{3}, 500 \mathrm{MHz}\right) \delta 1.04(\mathrm{~s}, \mathrm{OH}), 1.17(\mathrm{~s}, 3 \mathrm{H}), 1.24(\mathrm{~s}, 3 \mathrm{H}), 1.37-1.48(\mathrm{~m}, 2 \mathrm{H}), 1.60-1.80(\mathrm{~m}, 4 \mathrm{H})$, 1.84-1.90 (m, 1H), $2.12(\mathrm{dd}, J=7.5,12.5 \mathrm{~Hz}, 1 \mathrm{H}), 2.25-2.28(\mathrm{~m}, 1 \mathrm{H}), 2.90(\mathrm{ddd}, J=7.0,12.5,17.0 \mathrm{~Hz}$, $1 \mathrm{H}), 2.96(\mathrm{dd}, J=6.5,17.0 \mathrm{~Hz}, 1 \mathrm{H}), 7.05(\mathrm{t}, J=6.5 \mathrm{~Hz}, 1 \mathrm{H}), 7.09(\mathrm{dt}, J=1.0,7.0 \mathrm{~Hz}, 1 \mathrm{H}), 7.13(\mathrm{t}, J=$ $7.5 \mathrm{~Hz}, 1 \mathrm{H}), 7.26(\mathrm{~m}, 1 \mathrm{H}) ;{ }^{13} \mathrm{C} \mathrm{NMR}\left(\mathrm{CDCl}_{3}, 125 \mathrm{MHz}\right) \delta 17.9,20.5,23.0,24.5,30.3,37.9,38.5,42.7$, 52.3, 72.4, 124.7, 125.4, 125.7, 129.2, 135.1, 148.9; HRMS (EI) $\mathrm{m} / z$ calcd for $\mathrm{C}_{16} \mathrm{H}_{22} \mathrm{O}(\mathrm{M})$ 230.1671, found 230.1682 .<smiles>CC12CCC[C@@H](O)[C@]1(C)CCc1ccccc12</smiles>

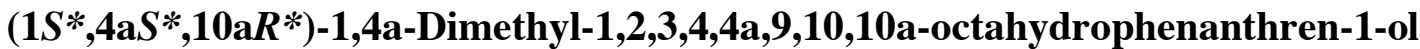

(5b $\beta$-OH) (Table 1): Column chromatography (hexane-EtOAc $=10: 1$ ); colorless oil; TLC, $R_{\mathrm{f}}=0.38$ (hexane-EtOAc = 4:1); IR (film) 3608, 3009, 2936, 1488, 1457, 1375, 1077, $1019 \mathrm{~cm}^{-1}$; ${ }^{1} \mathrm{H}$ NMR $\left(\mathrm{CDCl}_{3}, 500 \mathrm{MHz}\right) \delta 1.05(\mathrm{~s}, \mathrm{OH}), 1.26(\mathrm{~s}, 3 \mathrm{H}), 1.31(\mathrm{~s}, 3 \mathrm{H}), 1.41-1.48(\mathrm{~m}, 3 \mathrm{H}), 1.60-1.66(\mathrm{~m}, 1 \mathrm{H})$, $1.75(\mathrm{dm}, J=13.5 \mathrm{~Hz}, 1 \mathrm{H}), 1.89(\mathrm{ddt}, J=7.5,10.5,13.0 \mathrm{~Hz}, 1 \mathrm{H}), 1.97(\mathrm{ttd}, J=3.5,7.0,14.0 \mathrm{~Hz}, 1 \mathrm{H})$, 2.00-2.06 (m, 1H), $2.23(\mathrm{dd}, J=1.5,13.0 \mathrm{~Hz}), 2.93(\mathrm{ddd}, J=8.0,10.5,17.5 \mathrm{~Hz}, 1 \mathrm{H}), 3.00(\mathrm{ddd}, J=2.0$, 8.0, $17.5 \mathrm{~Hz}, 1 \mathrm{H}), 7.06$ (brt, $J=6.5 \mathrm{~Hz}, 1 \mathrm{H}), 7.08$ (dt, $J=1.0,7.0 \mathrm{~Hz}, 1 \mathrm{H}), 7.13$ (brt, $J=7.0 \mathrm{~Hz}, 1 \mathrm{H}$ ); ${ }^{13} \mathrm{C} \mathrm{NMR}\left(\mathrm{CDCl}_{3}, 125 \mathrm{MHz}\right) \delta 17.8,18.4,24.4,29.3,30.8,37.4,38.1,40.7,48.5,72.2,124.1,125.3$, 125.7, 129.0, 135.0, 149.3; HRMS (EI) $\mathrm{m} / z$ calcd for $\mathrm{C}_{16} \mathrm{H}_{22} \mathrm{O}$ (M) 230.1671, found 230.1682. 
<smiles>Cc1ccc2c(c1)C1(C)CCC[C@@H](C)[C@H]1CC2</smiles>

$(1 R *, 4 \mathrm{a} S *, 10 \mathrm{a} R *)-1,4 \mathrm{a}, 6-$ Trimethyl-1,2,3,4,4a,9,10,10a-octahydrophenanthren-

1-ol (5c $\alpha-O H)$ (Table 1): Column chromatography (hexane-EtOAc = 2:1); colorless oil; TLC, $R_{\mathrm{f}}=$ 0.25 (hexane-EtOAc = 4:1), 0.50 (hexane-EtOAc = 30:1); IR (film) 3596, 3379, 3007, 2929, 2856, 1499, 1457, 1164, $1093 \mathrm{~cm}^{-1}$; ${ }^{1} \mathrm{H}$ NMR $\left(\mathrm{CDCl}_{3}, 300 \mathrm{MHz}\right) \delta 1.04(\mathrm{~s}, \mathrm{OH}), 1.16$ (s, 3H), 1.23 (s, 3H), $1.38-1.80(\mathrm{~m}, 6 \mathrm{H}), 1.84-1.90(\mathrm{dm}, J=12.3 \mathrm{~Hz}, 1 \mathrm{H}), 2.02-2.13(\mathrm{~m}, 1 \mathrm{H}), 2.23-2.30(\mathrm{~m}, 1 \mathrm{H}), 2.30(\mathrm{~s}$, $3 \mathrm{H}), 2.78-2.30(\mathrm{~m}, 2 \mathrm{H}), 6.90-6.97(\mathrm{~m}, 2 \mathrm{H}), 7.06(\mathrm{~s}, 1 \mathrm{H})$; HRMS (EI) $\mathrm{m} / z$ calcd for $\mathrm{C}_{17} \mathrm{H}_{24} \mathrm{O}(\mathrm{M})$ 244.1827, found 244.1833 .<smiles>Cc1ccc2c(c1)C1(C)CCC[C@H](O)[C@H]1CC2</smiles>

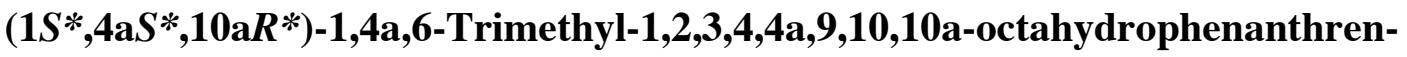

1-ol (5c $\beta$-OH) (Table 1): Column chromatography (hexane-EtOAc $=5: 1$ ); white solid; TLC, $R_{\mathrm{f}}=0.38$ (hexane-EtOAc = 4:1); IR (film) 3607, 3600-3250 (br), 3042, 3007, 2940, 1500, 1456, 1375, $937 \mathrm{~cm}^{-1}$; ${ }^{1} \mathrm{H}$ NMR $\left(\mathrm{CDCl}_{3}, 500 \mathrm{MHz}\right) \delta 1.06(\mathrm{~s}, \mathrm{OH}), 1.26(\mathrm{~s}, 3 \mathrm{H}), 1.31(\mathrm{~s}, 3 \mathrm{H}), 1.40-1.47(\mathrm{~m}, 3 \mathrm{H}), 1.60-1.64(\mathrm{~m}$, $1 \mathrm{H}), 1.75(\mathrm{dm}, J=14.0 \mathrm{~Hz}, 1 \mathrm{H}), 1.86(\mathrm{ddt}, J=7.5,10.5,13.0 \mathrm{~Hz}, 1 \mathrm{H}), 1.97(\mathrm{dtt}, J=3.5,7.0,13.5 \mathrm{~Hz}$, $1 \mathrm{H}), 1.98-2.05(\mathrm{~m}, 1 \mathrm{H}), 2.30(\mathrm{~s}, 3 \mathrm{H}), 2.30-2.34(\mathrm{~m}, 1 \mathrm{H}), 2.83-2.90(\mathrm{~m}, 1 \mathrm{H}), 2.94-2.98(\mathrm{~m}, 1 \mathrm{H}), 6.91$ $(\mathrm{dd}, J=1.0,8.0 \mathrm{~Hz}, 1 \mathrm{H}), 6.95(\mathrm{~d}, J=8.0 \mathrm{~Hz}, 1 \mathrm{H}), 7.07(\mathrm{~s}, 1 \mathrm{H}) ;{ }^{13} \mathrm{C}$ NMR $\left(\mathrm{CDCl}_{3}, 125 \mathrm{MHz}\right) \delta 17.9$, 18.4, 21.3, 24.4, 28.9, 30.8, 37.4, 38.1, 40.7, 48.7, 72.3, 124.6, 126.2, 128.9, 131.8, 134.9, 149.1; HRMS (EI) $\mathrm{m} / z$ calcd for $\mathrm{C}_{17} \mathrm{H}_{24} \mathrm{O}(\mathrm{M}) 244.1827$, found 244.1837 .<smiles>Cc1ccc2c(c1)CC[C@@H]1[C@@H](C)CCCC21C</smiles>

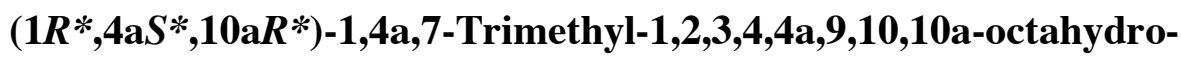

phenanthren-1-ol (5d $\mathbf{\alpha - O H})$ (Table 1): Column chromatography (hexane-EtOAc = 5:1); white solid; TLC, $R_{\mathrm{f}}=0.32$ (hexane-EtOAc = 10:1); IR (film) 3596, 3007, 2930, 2856, 1496, 1457, 1380, 891, 820 $\mathrm{cm}^{-1} ;{ }^{1} \mathrm{H}$ NMR $\left(\mathrm{CDCl}_{3}, 500 \mathrm{MHz}\right) \delta 1.04(\mathrm{~s}, \mathrm{OH}), 1.15(\mathrm{~s}, 3 \mathrm{H}), 1.24(\mathrm{~s}, 3 \mathrm{H}), 1.36-1.45(\mathrm{~m}, 2 \mathrm{H}), 1.59$ $(\mathrm{dd}, J=2.0,13.0 \mathrm{~Hz}, 1 \mathrm{H}), 1.63-1.80(\mathrm{~m}, 3 \mathrm{H}), 1.87(\mathrm{dm}, J=12.5 \mathrm{~Hz}, 1 \mathrm{H}), 2.10(\mathrm{ddt}, J=2.0,7.0,12.5$ $\mathrm{Hz}, 1 \mathrm{H}), 2.23-2.27(\mathrm{~m}, 1 \mathrm{H}), 2.27(\mathrm{~s}, 3 \mathrm{H}), 2.82-2.95(\mathrm{~m}, 2 \mathrm{H}), 6.88(\mathrm{~s}, 1 \mathrm{H}), 6.95(\mathrm{~d}, J=8.0 \mathrm{~Hz}, 1 \mathrm{H})$, $7.15(\mathrm{~d}, J=8.0 \mathrm{~Hz}, 1 \mathrm{H}) ;{ }^{13} \mathrm{C} \mathrm{NMR}\left(\mathrm{CDCl}_{3}, 125 \mathrm{MHz}\right) \delta 17.9,20.6,20.8,20.8,22.9,24.5,30.2,38.1$, 38.2, 42.7, 52.5, 72.4, 124.6, 126.6, 129.7, 134.8, 134.9, 146.1; HRMS (EI) $\mathrm{m} / z$ calcd for $\mathrm{C}_{17} \mathrm{H}_{24} \mathrm{O}(\mathrm{M})$ 244.1827, found 244.1837 .<smiles>Cc1ccc2c(c1)CC[C@@H]1[C@@H](O)CCC[C@]21C</smiles>

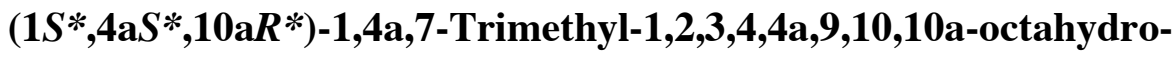


phenanthren-1-ol (5d $\beta$-OH) (Table 1): Column chromatography (hexane-EtOAc $=10: 1)$; white solid; TLC, $R_{\mathrm{f}}=0.49$ (hexane-EtOAc $=4: 1$ ); IR (film) 3608, 3007, 2934, 2859, 1459, 1456, 1375, 934, 879, $820 \mathrm{~cm}^{-1} ;{ }^{1} \mathrm{H}$ NMR $\left(\mathrm{CDCl}_{3}, 500 \mathrm{MHz}\right) \delta 1.06(\mathrm{~s}, \mathrm{OH}), 1.26(\mathrm{~s}, 3 \mathrm{H}), 1.30(\mathrm{~s}, 3 \mathrm{H}), 1.38-1.47(\mathrm{~m}$, $3 \mathrm{H}), 1.62(\mathrm{ttd}, J=3.5,7.0,14.0 \mathrm{~Hz}, 1 \mathrm{H}), 1.74(\mathrm{dm}, J=13.5 \mathrm{~Hz}, 1 \mathrm{H}), 1.87(\mathrm{ddt}, J=7.5,10.5,13.0 \mathrm{~Hz}$, $1 \mathrm{H}), 1.96(\mathrm{ttd}, J=3.5,7.0,14.0 \mathrm{~Hz}, 1 \mathrm{H}), 1.99-2.05(\mathrm{~m}, 1 \mathrm{H}), 2.28(\mathrm{~s}, 3 \mathrm{H}), 2.28-2.33$ (m, 1H), 2.87 (ddd, $J=8.0,10.5,18.0 \mathrm{~Hz}, 1 \mathrm{H}), 2.96(\mathrm{ddd}, J=2.0,7.5,18.0 \mathrm{~Hz}, 1 \mathrm{H}), 6.89(\mathrm{~s}, 1 \mathrm{H}), 6.95(\mathrm{~d}, J=8.0 \mathrm{~Hz}, 1 \mathrm{H})$, $7.16(\mathrm{~d}, J=8.0 \mathrm{~Hz}, 1 \mathrm{H}) ;{ }^{13} \mathrm{C} \mathrm{NMR}\left(\mathrm{CDCl}_{3}, 125 \mathrm{MHz}\right) \delta 17.9,18.4,20.8,24.4,29.2,30.8,37.1,38.2$, 40.8, 48.8, 72.2, 124.0, 126.5, 129.5, 134.7, 134.8, 146.5; HRMS (EI) $\mathrm{m} / z$ calcd for $\mathrm{C}_{17} \mathrm{H}_{24} \mathrm{O}(\mathrm{M})$ 244.1827, found 244.1837.

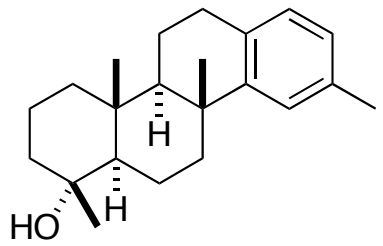

$\left(1 R^{*}, 4 \mathrm{a} R^{*}, 4 \mathrm{~b} R^{*}, 10 \mathrm{~b} R^{*}, 12 \mathrm{a} R^{*}\right)-1,4 \mathrm{a}, 9,10 \mathrm{~b}-$ Tetramethyl1,2,3,4,4a,4b,5,6,10b,11,12,12a-dodecahydrochrysen-1-ol (8a $\alpha-O H) \quad($ Table 2): Column chromatography (hexane-EtOAc $=5: 1$ ); white solid; TLC $R_{\mathrm{f}}=0.31$ (hexane-EtOAc $\left.=4: 1\right)$; IR (film) 3593, 3550-3300 (br), 2930, 2855, 1500, 1458, 1387, 1096, $1062 \mathrm{~cm}^{-1} ;{ }^{1} \mathrm{H} \mathrm{NMR}\left(\mathrm{CDCl}_{3}, 500 \mathrm{MHz}\right) \delta$ 0.86-0.93 (m, 1H), $0.89(\mathrm{~s}, 3 \mathrm{H}), 1.10-2.05(12 \mathrm{H}), 1.17(\mathrm{~s}, 3 \mathrm{H}), 1.20(\mathrm{~s}, 3 \mathrm{H}), 2.29(\mathrm{~s}, 3 \mathrm{H}), 2.43(\mathrm{dd}, J=$ 3.0, $9.5 \mathrm{~Hz}, 1 \mathrm{H}), 2.79$ (ddd, $J=7.5,11.5,17.0 \mathrm{~Hz}, 1 \mathrm{H}), 2.89(\mathrm{dd}, J=6.0,17.0 \mathrm{~Hz}, 1 \mathrm{H}), 6909$ (dd, $J=$ 1.5, 7.5 Hz, 1H), $6.92(\mathrm{~d}, J=7.5 \mathrm{~Hz}, 1 \mathrm{H}), 7.06(\mathrm{~s}, 1 \mathrm{H}) ;{ }^{13} \mathrm{C} \mathrm{NMR}\left(\mathrm{CDCl}_{3}, 125 \mathrm{MHz}\right) \delta 15.7,18.0,18.3$, 19.8, 21.3, 22.7, 26.1, 30.4, 38.1, 38.4, 39.0, 39.9, 42.9, 55.0, 57.7, 72.3, 125.1, 126.1, 128.7, 131.8, 135.0, 149.9; HRMS (EI) $\mathrm{m} / z$ calcd for $\mathrm{C}_{22} \mathrm{H}_{32} \mathrm{O}(\mathrm{M}) 312.2453$, found 312.2441 .<smiles>Cc1ccc2c(c1)C1(C)CCC3C(C)(CCC[C@@]3(C)O)C1CC2</smiles>

$(1 S *, 4 \mathrm{a} R *, 4 \mathrm{~b} R *, 10 \mathrm{~b} R *, 12 \mathrm{a} R *)-1,4 \mathrm{a}, 9,10 \mathrm{~b}-$ Tetramethyl1,2,3,4,4a,4b,5,6,10b,11,12,12a-dodecahydrochrysen-1-ol (8aß-OH) (Table 2): Column chromatography (hexane-EtOAc $=10: 1$ ); amorphous solid; TLC (hexane-EtOAc $=4: 1) R_{\mathrm{f}}=0.50$; IR (film) 3609, 3006, 2927, 2854, 1716, 1500, 1457, 1379, $1262 \mathrm{~cm}^{-1} ;{ }^{1} \mathrm{H} \mathrm{NMR}\left(\mathrm{CDCl}_{3}, 500 \mathrm{MHz}\right) \delta 0.86-$ $0.93(\mathrm{~m}, 1 \mathrm{H}), 0.99(\mathrm{dd}, J=2.5,12.5 \mathrm{~Hz}, 1 \mathrm{H}), 1.08(\mathrm{~s}, 3 \mathrm{H}), 1.20(\mathrm{~s}, 3 \mathrm{H}), 1.23(\mathrm{~s}, 3 \mathrm{H}), 1.26(\mathrm{dd}, J=2.5$, $12.5 \mathrm{~Hz}, 1 \mathrm{H}), 1.34-1.90(\mathrm{~m}, 10 \mathrm{H}), 2.29(\mathrm{~s}, 3 \mathrm{H}), 2.44(\mathrm{dt}, J=3.5,13.0 \mathrm{~Hz}, 1 \mathrm{H}), 2.78$ (ddd, $J=7.5,11.5$, $16.5 \mathrm{~Hz}, 1 \mathrm{H}), 2.90(\mathrm{ddm}, J=6.5,16.5 \mathrm{~Hz}, 1 \mathrm{H}), 6.89(\mathrm{dd}, J=1.0,7.5 \mathrm{~Hz}, 1 \mathrm{H}), 6.92(\mathrm{~d}, J=7.5 \mathrm{~Hz}, 1 \mathrm{H})$, $7.05(\mathrm{~s}, 1 \mathrm{H}) ;{ }^{13} \mathrm{C} \mathrm{NMR}\left(\mathrm{CDCl}_{3}, 125 \mathrm{MHz}\right) \delta$ 15.7, 17.8, 17.9, 18.3, 21.3, 26.1, 30.2, 30.6, 37.5, 37.9, 39.2, 40.2, 40.9, 54.4, 54.5, 72.2, 124.9, 126.1, 128.7, 131.9, 134.9, 150.2; HRMS (EI) $\mathrm{m} / z$ calcd for $\mathrm{C}_{22} \mathrm{H}_{32} \mathrm{O}(\mathrm{M}) 312.2453$, found 312.2441 .

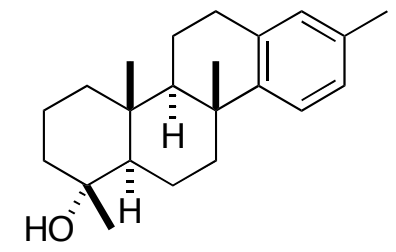

$\left(1 R^{*}, 4 \mathrm{a} R^{*}, 4 \mathrm{~b} R^{*}, 10 \mathrm{~b} R^{*}, 12 \mathrm{a} R^{*}\right)-1,4 \mathrm{a}, 8,10 \mathrm{~b}-$ Tetramethyl1,2,3,4,4a,4b,5,6,10b,11,12,12a-dodecahydrochrysen-1-ol $\quad(8 \mathrm{~b} \alpha-\mathrm{OH}) \quad($ Table 2$): \quad$ Column chromatography (hexane-EtOAc $=5: 1$ ); amorphous solid; TLC, $R_{\mathrm{f}}=0.36$ (hexane-EtOAc $\left.=4: 1\right)$; IR (film) 3598, 3600-3250 (br), 3006, 2931, 2855, 1459, 1387, 1096, $820 \mathrm{~cm}^{-1} ;{ }^{1} \mathrm{H}$ NMR $\left(\mathrm{CDCl}_{3}, 500\right.$ MHz) $\delta$ 0.84-2.43 (m, 14H), 0.90 (s, 3H), 1.17 (s, 3H), 1.19 (s, 3H), 2.27 (s, 3H), 2.77-2.92 (m, 2H), $6.86(\mathrm{~s}, 1 \mathrm{H}), 6.95(\mathrm{~d}, J=8.5 \mathrm{~Hz}, 1 \mathrm{H}), 7.15(\mathrm{~d}, J=8.5 \mathrm{~Hz}, 1 \mathrm{H}) ;{ }^{13} \mathrm{C} \mathrm{NMR}\left(\mathrm{CDCl}_{3}, 125 \mathrm{MHz}\right) \delta 15.7$, 18.0, 18.3, 19.8, 20.8, 22.7, 26.1, 30.7, 37.8, 38.3, 39.0, 40.0, 42.9, 55.1, 57.7, 72.3, 124.6, 126.6, 129.4, 
134.6, 134.8, 147.2; HRMS (EI) $m / z$ calcd for $\mathrm{C}_{22} \mathrm{H}_{32} \mathrm{O}$ (M) 312.2453, found 312.2441.<smiles>Cc1ccc2c(c1)CCC1C2(C)CCC2[C@H](C)CCCC21C</smiles>

$(1 S *, 4 \mathrm{a} R *, 4 \mathrm{~b} R *, 10 \mathrm{~b} R *, 12 \mathrm{a} R *)-1,4 \mathrm{a}, 8,10 \mathrm{~b}-$ Tetramethyl1,2,3,4,4a,4b,5,6,10b,11,12,12a-dodecahydrochrysen-1-ol $\quad(8 \mathrm{~b} \boldsymbol{\beta}-\mathrm{OH}) \quad$ (Table 2): Column chromatography (hexane-EtOAc $=10: 1$ ); amorphous solid; TLC, $R_{\mathrm{f}}=0.56$ (hexane-EtOAc $=4: 1$ ); IR (film) 3608, 3006, 2927, 1457, 1379, 1262, 1092, 1024, $820 \mathrm{~cm}^{-1} ;{ }^{1} \mathrm{H} \mathrm{NMR}\left(\mathrm{CDCl}_{3}, 500 \mathrm{MHz}\right) \delta 0.83-$ $0.94(\mathrm{~m}, 1 \mathrm{H}), 0.99(\mathrm{dd}, J=2.5,12.5 \mathrm{~Hz}, 1 \mathrm{H}), 1.07(\mathrm{~s}, 3 \mathrm{H}), 1.20(\mathrm{~s}, 3 \mathrm{H}), 1.22(\mathrm{~s}, 3 \mathrm{H}), 1.26(\mathrm{dd}, J=2.0$, $12.0 \mathrm{~Hz}, 1 \mathrm{H}), 1.36-1.89(\mathrm{~m}, 10 \mathrm{H}), 2.27(\mathrm{~s}, 3 \mathrm{H}), 2.43(\mathrm{dt}, J=3.0,12.5 \mathrm{~Hz}, 1 \mathrm{H}), 2.79$ (ddd, $J=7.5,11.5$, $17.5 \mathrm{~Hz}, 1 \mathrm{H}), 2.90(\mathrm{dd}, J=7.0,17.5 \mathrm{~Hz}, 1 \mathrm{H}), 6.85(\mathrm{~s}, 1 \mathrm{H}), 6.94(\mathrm{~d}, J=8.0 \mathrm{~Hz}, 1 \mathrm{H}), 7.14(\mathrm{~d}, J=8.0 \mathrm{~Hz}$, $1 \mathrm{H}) ;{ }^{13} \mathrm{C}$ NMR $\left(\mathrm{CDCl}_{3}, 125 \mathrm{MHz}\right) \delta 15.7,17.8(2 \mathrm{C}), 18.3,20.8,26.2,30.6(2 \mathrm{C}), 37.5,37.7,39.2,40.2$, $40.9,54.5$ (2C), 72.2, 124.4, 126.6, 129.4, 134.6, 134.9, 147.5; HRMS (EI) $\mathrm{m} / z$ calcd for $\mathrm{C}_{22} \mathrm{H}_{32} \mathrm{O}(\mathrm{M})$ 312.2453 , found 312.2463 .<smiles>Cc1ccc2c(c1)C1(C)CCC[C@H](O)[C@]1(C)CC2</smiles>

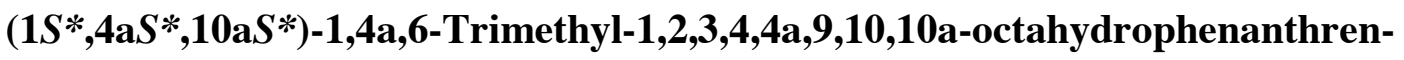

1-ol (11aß-OH) (Table 3): Column chromatography (hexane-EtOAc $=5: 1$ ); white solid; TLC, $R_{\mathrm{f}}=$ 0.23 (hexane-EtOAc = 4:1); IR (film) 3598, 3007, 2930, 2868, 1505, 1457, 1376, 1084, $813 \mathrm{~cm}^{-1} ;{ }^{1} \mathrm{H}$ NMR $\left(\mathrm{CDCl}_{3}, 500 \mathrm{MHz}\right) \delta 1.07(\mathrm{~s}, 3 \mathrm{H}), 1.37-2.00(\mathrm{~m}, 9 \mathrm{H}), 1.50(\mathrm{~s}, 3 \mathrm{H}), 2.30(\mathrm{~s}, 3 \mathrm{H}), 2.73-2.85(\mathrm{~m}$, $2 \mathrm{H}), 6.89(\mathrm{~d}, J=8.0 \mathrm{~Hz}, 1 \mathrm{H}), 6.92(\mathrm{~d}, J=8.0 \mathrm{~Hz}, 1 \mathrm{H}), 7.09(\mathrm{~s}, 1 \mathrm{H}) ;{ }^{13} \mathrm{C} \mathrm{NMR}\left(\mathrm{CDCl}_{3}, 125 \mathrm{MHz}\right) \delta 19.4$, 21.2, 21.4, 29.1, 29.3 (2C), 30.5, 37.0, 38.5, 52.2, 73.8, 126.0, 126.4, 128.4, 133.0, 135.0, 145.9; HRMS (EI) $\mathrm{m} / \mathrm{z}$ calcd for $\mathrm{C}_{17} \mathrm{H}_{24} \mathrm{O}$ (M) 244.1827, found 244.1837 .<smiles>Cc1ccc2c(c1)CC[C@H]1[C@@H](O)CCC[C@]21C</smiles>

\section{$\left(1 R^{*}, 4 \mathrm{a} S^{*}, 10 \mathrm{a} S^{*}\right)-1,4 \mathrm{a}, 7-T r i m e t h y l-1,2,3,4,4 \mathrm{a}, 9,10,10 \mathrm{a}-\mathrm{octahydro}-$}

phenanthren-1-ol (11ba-OH) (Table 3): Column chromatography (hexane-EtOAc = 10:1); colorless oil; TLC, $R_{\mathrm{f}}=0.52$ (hexane-EtOAc $=4: 1$ ); IR (film) 3568, 3006, 2927, 2858, 1719, 1456, 1379, 1261, $1102,818 \mathrm{~cm}^{-1} ;{ }^{1} \mathrm{H}$ NMR $\left(\mathrm{CDCl}_{3}, 500 \mathrm{MHz}\right) \delta 1.17(\mathrm{~s}, 3 \mathrm{H}), 1.23(\mathrm{~s}, 3 \mathrm{H}), 1.37-1.68(\mathrm{~m}, 6 \mathrm{H}), 2.14-2.31$ $(\mathrm{m}, 2 \mathrm{H}), 2.27(\mathrm{~s}, 3 \mathrm{H}), 2.44(\mathrm{dd}, J=3.0,14.5 \mathrm{~Hz}, 1 \mathrm{H}), 2.77(\mathrm{ddd}, J=3.0,8.5,17.5 \mathrm{~Hz}, 1 \mathrm{H}), 3.11-3.19$ $(\mathrm{m}, 1 \mathrm{H}), 6.90(\mathrm{~s}, 1 \mathrm{H}), 6.95(\mathrm{~d}, J=7.5 \mathrm{~Hz}, 1 \mathrm{H}), 7.20(\mathrm{~d}, J=7.5 \mathrm{~Hz}, 1 \mathrm{H}) ;{ }^{13} \mathrm{C} \mathrm{NMR}\left(\mathrm{CDCl}_{3}, 125 \mathrm{MHz}\right) \delta$ 18.1, 18.3, 20.9, 26.3, 28.8, 35.2, 36.7, 37.4, 41.7, 48.6, 73.0, 124.3, 126.8, 130.1, 135.3, 138.1, 138.4; HRMS (EI) $m / z$ calcd for $\mathrm{C}_{17} \mathrm{H}_{24} \mathrm{O}(\mathrm{M}) 244.1827$, found 244.1819 .<smiles>Cc1ccc2c(c1)CC[C@H]1[C@@H](O)CCCC21C</smiles> 
phenanthren-1-ol (11b $\beta$-OH) (Table 3): Column chromatography (hexane-EtOAc = 5:1); colorless oil; TLC, $R_{\mathrm{f}}=0.31$ (hexane-EtOAc $=4: 1$ ); IR (film) 3596, 3550-3250 (br), 3007, 2932, 2868, 1499, 1457, 1377, $822 \mathrm{~cm}^{-1} ;{ }^{1} \mathrm{H}$ NMR $\left(\mathrm{CDCl}_{3}, 500 \mathrm{MHz}\right) \delta 1.08(\mathrm{~s}, 3 \mathrm{H}), 1.37-1.68(\mathrm{~m}, 6 \mathrm{H}), 1.49(\mathrm{~s}, 3 \mathrm{H})$, 1.72-2.00 (m, 4H), $2.27(\mathrm{~s}, 3 \mathrm{H}), 2.73-2.86(\mathrm{~m}, 2 \mathrm{H}), 6.85(\mathrm{~s}, 1 \mathrm{H}), 6.96(\mathrm{~d}, J=8.0 \mathrm{~Hz}, 1 \mathrm{H}), 7.18(\mathrm{~d}, J=$ $8.0 \mathrm{~Hz}, 1 \mathrm{H}) ;{ }^{13} \mathrm{C} \mathrm{NMR}\left(\mathrm{CDCl}_{3}, 125 \mathrm{MHz}\right) \delta 19.3,20.8,21.2,29.3,29.5,29.7,30.5,37.1,38.3,52.2$, 73.8, 125.8, 126.7, 129.1, 134.5, 135.9, 143.2; HRMS (EI) $m / z$ calcd for $\mathrm{C}_{17} \mathrm{H}_{24} \mathrm{O}$ (M) 244.1827, found 244.1837.

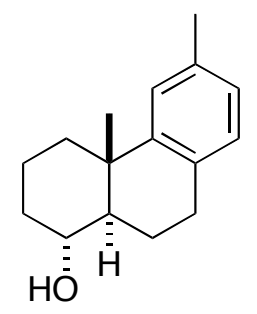

$\left(1 R^{*}, 4 \mathrm{a} S *, 10 \mathrm{a} R *\right)-4 \mathrm{a}, 6$-dimethyl-1,2,3,4,4a,9,10,10a-octahydrophenanthren-1-ol $(\mathbf{1 5 a \alpha})\left(\right.$ Entry 1, Table 4): Column chromatography (hexane-EtOAc = 5:1); colorless oil; $\mathrm{TLC}, R_{\mathrm{f}}=$ 0.21 (hexane-EtOAc = 4:1); ${ }^{1} \mathrm{H}$ NMR $\left(\mathrm{CDCl}_{3}, 500 \mathrm{MHz}\right) \delta 1.09(\mathrm{~s}, 1 \mathrm{H}), 1.21-1.31(\mathrm{~m}, 1 \mathrm{H}), 1.37-1.44$ $(\mathrm{m}, 3 \mathrm{H}), 1.59$ (ddt, $J=7.0,11.5,13.0 \mathrm{~Hz}, 1 \mathrm{H}), 1.70(\mathrm{tq}, J=4.0,14.0 \mathrm{~Hz}, 1 \mathrm{H}), 1.76-1.81(\mathrm{~m}, 1 \mathrm{H}), 2.07-$ $2.14(\mathrm{~m}, 1 \mathrm{H}), 2.18-2.26(\mathrm{~m}, 2 \mathrm{H}), 2.30(\mathrm{~s}, 3 \mathrm{H}), 2.80-2.91(\mathrm{~m}, 2 \mathrm{H}), 3.60(\mathrm{dt}, J=4.5,11.0 \mathrm{~Hz}, 1 \mathrm{H}), 6.92$ $(\mathrm{dd}, J=1.5,7.5 \mathrm{~Hz}, 1 \mathrm{H}), 6.97(\mathrm{~d}, J=7.5 \mathrm{~Hz}, 1 \mathrm{H}), 7.07(\mathrm{~s}, 1 \mathrm{H}) ;{ }^{13} \mathrm{C} \mathrm{NMR}\left(\mathrm{CDCl}_{3}, 125 \mathrm{MHz}\right) \delta 19.6$, 20.6, 21.3, 23.2, 28.9, 36.1, 37.1, 38.2, 49.7, 70.4, 125.2, 126.4, 129.2, 132.2, 134.9, 147.2.<smiles>Cc1ccc2c(c1)C1(C)CCCC(O)C1CC2</smiles>

$\left(1 S^{*}, 4 \mathrm{a} S *, 10 \mathrm{a} R *\right)-4 \mathrm{a}, 6-D i m e t h y l-1,2,3,4,4 \mathrm{a}, 9,10,10 \mathrm{a}-0 \mathrm{ctahydrophenanthren-1-ol}$ (15aß) (Entry 1, Table 4): Column chromatography (hexane-EtOAc = 10:1); amorphous solid; TLC, $R_{\mathrm{f}}=0.30$ (hexane-EtOAc = 4:1); IR (film) 3619, 3008, 2933, 2870, 1499, 1455, 1075, $1000 \mathrm{~cm}^{-1} ;{ }^{1} \mathrm{H}$ NMR $\left(\mathrm{CDCl}_{3}, 500 \mathrm{MHz}\right) \delta 1.32(\mathrm{~s}, 3 \mathrm{H}), 1.43(\mathrm{dt}, J=3.0,13.0 \mathrm{~Hz}, 1 \mathrm{H}), 1.53-1.67(\mathrm{~m}, 4 \mathrm{H}), 1.88(\mathrm{dm}, J$ $=14.0 \mathrm{~Hz}, 1 \mathrm{H}), 2.00(\mathrm{tq}, J=3.5,13.5 \mathrm{~Hz}, 1 \mathrm{H}), 2.07-2.17(\mathrm{~m}, 1 \mathrm{H}), 2.30(\mathrm{~s}, 3 \mathrm{H}), 2.30-2.34(\mathrm{~m}, 1 \mathrm{H})$, 2.9--2.94 (m, 2H), $4.03(\mathrm{~s}, 1 \mathrm{H}), 6.91(\mathrm{~d}, J=7.5 \mathrm{~Hz}, 1 \mathrm{H}), 6.95(\mathrm{~d}, J=7.5 \mathrm{~Hz}, 1 \mathrm{H}), 7.06(\mathrm{~s}, 1 \mathrm{H}) ;{ }^{13} \mathrm{C}$ $\mathrm{NMR}\left(\mathrm{CDCl}_{3}, 125 \mathrm{MHz}\right) \delta 17.3,21.3,22.9,25.4,29.1,33.6,36.8,37.8,44.9,72.3,124.7,126.3,129.1$, 131.8, 135.0, 148.7; HRMS (EI) $\mathrm{m} / z$ calcd for $\mathrm{C}_{16} \mathrm{H}_{22} \mathrm{O}(\mathrm{M}) 230.1671$ found 230.1682 .

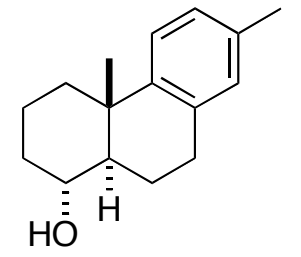

$\left(1 R *, 4 a S^{*}, 10 \mathrm{a} R *\right)-4 a, 7-D i m e t h y l-1,2,3,4,4 \mathrm{a}, 9,10,10 \mathrm{a}-$ octahydrophenanthren-1-ol (15bo) (Entry 2, Table 4): Containing a small amount of 5-Me-regioisomer; column chromatography (hexane-EtOAc $=5: 1$ ); colorless oil; TLC, $R_{\mathrm{f}}=0.21$ (hexane-EtOAc $\left.=4: 1\right) ;{ }^{1} \mathrm{H}$ NMR $\left(\mathrm{CDCl}_{3}, 300\right.$ $\mathrm{MHz}) \delta$ 0.83-1.77 (m, 6H), $1.08(\mathrm{~s}, 3 \mathrm{H}$ for 7-Me-regioisomer), 1.26 (s, 3H for 5-Me-regioisomer), 2.07-2.33 (m, 3H), 2.28 (s, 3H for 7-Me-regioisomer), 2.51 (s, 3H for 5-Me-regioisomer), 2.71-2.96 (m, 2H), $3.66(\mathrm{td}, J=4.2,10.5 \mathrm{~Hz}, 1 \mathrm{H}), 6.90(\mathrm{~s}, 1 \mathrm{H}), 6.96(\mathrm{dm}, J=7.5 \mathrm{~Hz}, 1 \mathrm{H}), 7.16(\mathrm{~d}, J=8.1 \mathrm{~Hz}, 1 \mathrm{H})$. 


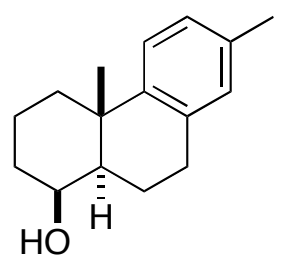

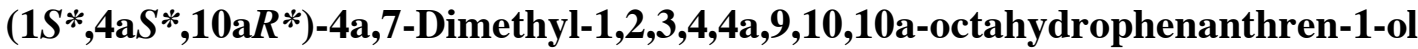
$(\mathbf{1 5 b} \beta)($ Entry 2, Table 4): Column chromatography (hexane-EtOAc $=10: 1$ ); amorphous solid; TLC, $R_{\mathrm{f}}=0.29$ (hexane-EtOAc = 4:1); IR (film) 3619, 3007, 2930, 2870, 1496, 1455, 1159, 1074, 1000, 821 $\mathrm{cm}^{-1} ;{ }^{1} \mathrm{H}$ NMR $\left(\mathrm{CDCl}_{3}, 500 \mathrm{MHz}\right) \delta 1.31(\mathrm{~s}, 3 \mathrm{H}), 1.41(\mathrm{dt}, J=3.0,13.0 \mathrm{~Hz}, 1 \mathrm{H}), 1.54-1.67(\mathrm{~m}, 4 \mathrm{H})$, 1.88 (brd, $J=14.5 \mathrm{~Hz}, 1 \mathrm{H}), 2.00$ (dq, $J=3.5,13.5 \mathrm{~Hz}, 1 \mathrm{H}), 2.07-2.17(\mathrm{~m}, 1 \mathrm{H}), 2.27$ (s, 3H), 2.27-2.32 (m, 1H), 2.92-2.94 (m, 2H), $4.03(\mathrm{~s}, 1 \mathrm{H}), 6.88(\mathrm{~s}, 1 \mathrm{H}), 6.96(\mathrm{~d}, J=7.5 \mathrm{~Hz}, 1 \mathrm{H}), 7.14(\mathrm{~d}, J=7.5 \mathrm{~Hz}$, $1 \mathrm{H}) ;{ }^{13} \mathrm{C}$ NMR $\left(\mathrm{CDCl}_{3}, 125 \mathrm{MHz}\right) \delta 17.3,20.8,22.9,25.5,29.4,33.7,36.5,37.9,45.0,72.2,124.1$, 126.6, 129.7, 134.8 (2C), 146.1; HRMS (EI) $\mathrm{m} / \mathrm{z}$ calcd for $\mathrm{C}_{16} \mathrm{H}_{22} \mathrm{O}$ (M) 230.1671 found, 230.1679.<smiles>CC(C)c1ccc2c(c1)CC[C@H]1[C@@H](C)CCC[C@@]21C</smiles>

$\left(1 R^{*}, 4 \mathrm{a} S^{*}, 10 \mathrm{a} R^{*}\right)-7-$ Isopropyl-1,4a-dimethyl-1,2,3,4,4a,9,10,10a-

octahydrophenanthren-1-ol (22) (Scheme 2): ${ }^{11}$ Column chromatography (hexane-EtOAc = 5:1); amorphous solid; TLC, $R_{\mathrm{f}}=0.20$ (hexane-EtOAc $\left.=4: 1\right) ;{ }^{1} \mathrm{H}$ NMR $\left(\mathrm{CDCl}_{3}, 500 \mathrm{MHz}\right) \delta 1.16(\mathrm{~s}, 3 \mathrm{H})$, $1.24(\mathrm{~d}, J=7.0 \mathrm{~Hz}, 6 \mathrm{H}), 1.24(\mathrm{~s}, 3 \mathrm{H}), 1.36-1.48(\mathrm{~m}, 2 \mathrm{H}), 1.60-1.79(\mathrm{~m}, 4 \mathrm{H}), 1.87(\mathrm{dm}, J=13.0 \mathrm{~Hz}$, $1 \mathrm{H}), 2.11(\mathrm{dd}, J=7.0,12.5 \mathrm{~Hz}, 1 \mathrm{H}), 2.26(\mathrm{dm}, J=13.0 \mathrm{~Hz}, 1 \mathrm{H}), 2.78-2.2 .92(\mathrm{~m}, 3 \mathrm{H}), 6.91(\mathrm{~s}, 1 \mathrm{H}), 7.01$ $(\mathrm{d}, J=8.0 \mathrm{~Hz}, 1 \mathrm{H}), 7.18(\mathrm{~d}, J=8.0 \mathrm{~Hz}, 1 \mathrm{H}) ;{ }^{13} \mathrm{C} \mathrm{NMR}\left(\mathrm{CDCl}_{3}, 125 \mathrm{MHz}\right) \delta 18.0,20.6,22.9,23.9,24.0$, 24.5, 30.4, 33.4, 38.0, 38.2, 42.7, 52.5, 72.5, 123.9, 124.6, 127.0, 134.8, 145.7, 146.4 .<smiles>CCCc1ccc2c(c1)CC[C@@H]1[C@@H](O)CCC[C@@]21C</smiles>

$\left(1 S^{*}, 4 \mathrm{a} S^{*}, 10 \mathrm{a} R *\right)$-7-Isopropyl-4a-methyl-1,2,3,4,4a,9,10,10a-octahydro-

phenanthren-1-ol (15c $\beta)$ (Scheme 2): ${ }^{12}$ Column chromatography (hexane-EtOAc $=5: 1$ ); amorphous solid; TLC, $R_{\mathrm{f}}=0.31$ (hexane-EtOAc $=4: 1$ ); IR (film) 3619, 3004, 2932, 2871, 1497, 1456, 1074, 998, $887,827 \mathrm{~cm}^{-1} ;{ }^{1} \mathrm{H}$ NMR $\left(\mathrm{CDCl}_{3}, 500 \mathrm{MHz}\right) \delta 1.23(\mathrm{~d}, J=7.0 \mathrm{~Hz}, 6 \mathrm{H}), 1.32(\mathrm{~s}, 3 \mathrm{H}), 1.40-1.46(\mathrm{~m}, 1 \mathrm{H})$, $1.52-1.56(\mathrm{~m}, 4 \mathrm{H}), 1.88(\mathrm{dd}, J=3.5,13.5 \mathrm{~Hz}, 1 \mathrm{H}), 2.00(\mathrm{tq}, J=3.5,13.5 \mathrm{~Hz}, 1 \mathrm{H}), 2.07-2.16(\mathrm{~m}, 1 \mathrm{H})$, 2.27-2.32 (m, 1H), 2.79-2.86 (m, 1H), 2.93-2.96 (m, 2H), $4.03(\mathrm{~d}, J=2.5 \mathrm{~Hz}, 1 \mathrm{H}), 6.91(\mathrm{~s}, 1 \mathrm{H}), 7.00$ $(\mathrm{dd}, J=1.5,8.0 \mathrm{~Hz}, 1 \mathrm{H}), 7.17(\mathrm{~d}, J=8.0 \mathrm{~Hz}, 1 \mathrm{H}) ;{ }^{13} \mathrm{C} \mathrm{NMR}\left(\mathrm{CDCl}_{3}, 125 \mathrm{MHz}\right) \delta 17.3,22.9,24.0(2 \mathrm{C})$, 25.5, 29.6, 33.4, 33.6, 36.5, 37.8, 44.9, 72.2, 123.8, 124.0, 127.0, 134.7, 145.6, 146.3; HRMS (EI) $\mathrm{m} / \mathrm{z}$ calcd for $\mathrm{C}_{18} \mathrm{H}_{26} \mathrm{O}$ (M) 258.1984, found 258.1974.

Total Synthesis of 24 from 15c: To a stirred suspension of 15c (50 mg, $0.19 \mathrm{mmol})$ and activated molecular sieves $4 \mathrm{~A}(190 \mathrm{mg})$ in $\mathrm{CH}_{2} \mathrm{Cl}_{2}(8 \mathrm{~mL})$ was added 4-methylmorpholine $N$-oxide (NMO, $67 \mathrm{mg}, 0.57 \mathrm{mmol}$ ) and tetra- $n$-propylammonium perruthenate(VII) (TPAP, $3.5 \mathrm{mg}, 0.01 \mathrm{mmol}$ ) successively at $0{ }^{\circ} \mathrm{C}$ and stirred for $3 \mathrm{~h}$ at room temperature. ${ }^{13}$ The resulting mixture was filtered through a short pad of silica gel and the resulting residue was washed with EtOAc. The filtrate was concentrated and the residue was purified by column chromatography on silica gel (eluent: hexaneEtOAc $=10: 1)$ to gave $\mathbf{2 3}(49 \mathrm{mg}, 0.19 \mathrm{mmol},>99 \%$ yield $)$ as white solid. To a solution of $\mathbf{2 3}$ (256 mg, 
$1.0 \mathrm{mmol})$ in THF $(10 \mathrm{~mL})$ was added $\mathrm{MeLi}\left(1.0 \mathrm{M}\right.$ in $\mathrm{Et}_{2} \mathrm{O}$, Kanto, $\left.1.3 \mathrm{~mL}, 1.3 \mathrm{mmol}\right)$ at $-78{ }^{\circ} \mathrm{C}$. After 30 minutes the reaction was quenched with aqueous $\mathrm{NH}_{4} \mathrm{Cl}$ and extracted with $\mathrm{Et}_{2} \mathrm{O}$ (twice). The combined organic layers were dried over anhydrous $\mathrm{MgSO}_{4}$ and concentrated in vacuo. The crude product was passed through a short pad of silica gel to give pure $\mathbf{2 4}$ (271 $\mathrm{mg}, 0.99 \mathrm{mmol},>99 \%$ yield) as an amorphous solid.<smiles>CCCc1ccc2c(c1)CC[C@@H]1C(=O)CCC[C@]21C</smiles>

\section{$(4 \mathrm{a} S *, 10 \mathrm{a} R *)-7-I s o p r o p y l-4 a-m e t h y l-2,3,4,4 a, 10,10 a-h e x a h y d r o-$}

phenanthren-1(9H)-one (23): ${ }^{14}$ Column chromatography (hexane-EtOAc = 10:1); white solid; TLC, $R_{\mathrm{f}}=0.50$ (hexane-EtOAc = 4:1); IR (film) 3006, 2961, 2872, $1709(\mathrm{C}=\mathrm{O}), 1497,1378,1315,1069,827$ $\mathrm{cm}^{-1} ;{ }^{1} \mathrm{H} \mathrm{NMR}\left(\mathrm{CDCl}_{3}, 500 \mathrm{MHz}\right) \delta 1.05(\mathrm{~s}, 3 \mathrm{H}), 1.23(\mathrm{~d}, J=7.0 \mathrm{~Hz}, 6 \mathrm{H}), 1.81(\mathrm{ddt}, J=6.5,12.3,14.0$ $\mathrm{Hz}, 1 \mathrm{H}), 1.89(\mathrm{dt}, J=5.0,13.5 \mathrm{~Hz}, 1 \mathrm{H}), 1.98-2.16(\mathrm{~m}, 3 \mathrm{H}), 2.34-2.43(\mathrm{~m}, 3 \mathrm{H}), 2.66(\mathrm{dd}, J=2.5,12.5$ $\mathrm{Hz}, 1 \mathrm{H}), 2.77-2.91(\mathrm{~m}, 3 \mathrm{H}), 6.94(\mathrm{~s}, 1 \mathrm{H}), 7.03(\mathrm{dd}, J=1.5,8.0 \mathrm{~Hz}, 1 \mathrm{H}), 7.21(\mathrm{~d}, J=8.0 \mathrm{~Hz}, 1 \mathrm{H}) ;{ }^{13} \mathrm{C}$ NMR $\left(\mathrm{CDCl}_{3}, 125 \mathrm{MHz}\right) \delta 17.8,22.9,23.9,24.2,29.1,33.8,37.3,41.2,42.5,55.7,124.4,125.2,127.6$, 134.9, 143.3, 146.6, $212.7(\mathrm{C}=\mathrm{O})$; HRMS (EI) $\mathrm{m} / \mathrm{z}$ calcd for $\mathrm{C}_{18} \mathrm{H}_{24} \mathrm{O}(\mathrm{M})$ 256.1827, found 256.1826.<smiles>CCCc1ccc2c(c1)CC[C@H]1[C@@H](O)CCC[C@]21C</smiles>

$\left(1 S *, 4 a S^{*}, 10 \mathrm{a} R *\right)-7-I s o p r o p y l-1,4 a-d i m e t h y l-1,2,3,4,4 a, 9,10,10 a-$

octahydrophenanthren-1-ol (24): ${ }^{11}$ Column chromatography (hexane-EtOAc = 10:1); amorphous solid; TLC, $R_{\mathrm{f}}=0.39$ (hexane-EtOAc $\left.=4: 1\right) ;{ }^{1} \mathrm{H} \mathrm{NMR}\left(\mathrm{CDCl}_{3}, 500 \mathrm{MHz}\right) \delta 1.24(\mathrm{~d}, J=7.0 \mathrm{~Hz}, 6 \mathrm{H})$, $1.25(\mathrm{~s}, 3 \mathrm{H}), 1.30(\mathrm{~s}, 3 \mathrm{H}), 1.39-1.48(\mathrm{~m}, 3 \mathrm{H}), 1.59-1.64(\mathrm{~m}, 1 \mathrm{H}), 1.73(\mathrm{dm}, J=13.5 \mathrm{~Hz}, 1 \mathrm{H}), 1.87$ (ddt, $J=7.5,10.5,13.0 \mathrm{~Hz}, 1 \mathrm{H}), 1.96(\mathrm{tq}, J=3.5,14.0 \mathrm{~Hz}, 1 \mathrm{H}), 1.99-2.05(\mathrm{~m}, 1 \mathrm{H}), 2.31(\mathrm{~d}, J=13.0 \mathrm{~Hz}, 1 \mathrm{H})$, 2.78-3.00 (m, 3H), $6.90(\mathrm{~s}, 1 \mathrm{H}), 6.98(\mathrm{~d}, J=8.0 \mathrm{~Hz}, 1 \mathrm{H}), 7.18(\mathrm{~d}, J=8.0 \mathrm{~Hz}, 1 \mathrm{H}) ;{ }^{13} \mathrm{C} \mathrm{NMR}\left(\mathrm{CDCl}_{3}\right.$, $125 \mathrm{MHz}) \delta 17.9,18.4,24.0(2 \mathrm{C}), 24.4,29.4,30.8,33.4,37.1,38.2,40.8,48.7,72.2,123.8,123.9$, $126.8,134.7,145.6,146.7$.

\section{Determination of the Relative Stereochemistry of Polycyclic Products}

The relative stereochemistry of $\mathbf{5} \mathbf{c} \boldsymbol{\alpha}$ and $\mathbf{5} \mathbf{c} \boldsymbol{\beta}$ was determined based on the X-ray diffraction analysis of $\mathbf{5} \mathbf{c} \boldsymbol{\beta}$ (Figure 2). Other relative stereochemistry of $\mathbf{5} \boldsymbol{\alpha}$ and $\mathbf{5} \boldsymbol{\beta}$ was determined as analogues of $\mathbf{5 c} \boldsymbol{\alpha}$ and $\mathbf{5} \mathbf{c} \boldsymbol{\beta}$ in comparison with their ${ }^{1} \mathrm{H}$ and ${ }^{13} \mathrm{C}$ NMR spectra.

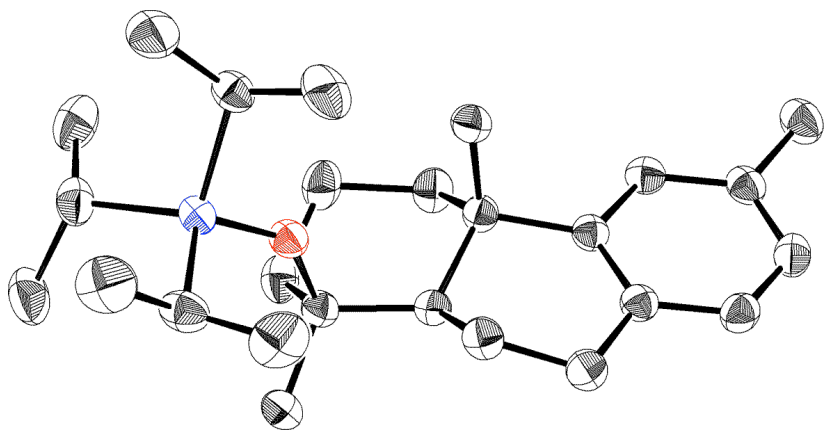


Figure 2. Molecular structure of $5 c \beta$. Carbon, silicon and oxygen atoms are shown in black, blue and red colors, respectively. All hydrogen atoms are omitted for clarity.

The relative stereochemistries of $\mathbf{8} \mathbf{a} \boldsymbol{\alpha}$ and $\mathbf{8 a} \boldsymbol{\beta}$ were determined based on the X-ray diffraction analyses of desilylated $\mathbf{8} \mathbf{a} \boldsymbol{\alpha}$ and desilylated $\mathbf{8 a} \boldsymbol{\beta}$ (Figures 3 and 4). Other relative stereochemistries of $\mathbf{8} \alpha$ and $\mathbf{8} \beta$ were determined as analogues of $\mathbf{8 a} \alpha$ and $\mathbf{8 a} \beta$ in comparison with their ${ }^{1} \mathrm{H}$ and ${ }^{13} \mathrm{C}$ NMR spectra.

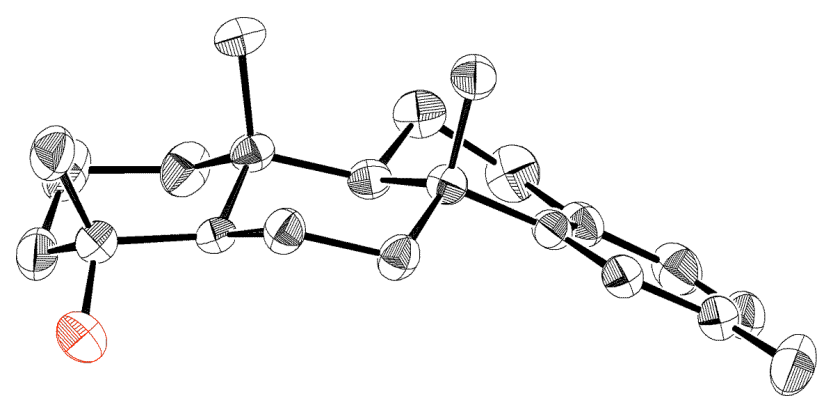

Figure 3. Molecular structure of desilylated 8a $\alpha$. Carbon and oxygen atoms are shown in black and red colors, respectively. All hydrogen atoms are omitted for clarity.

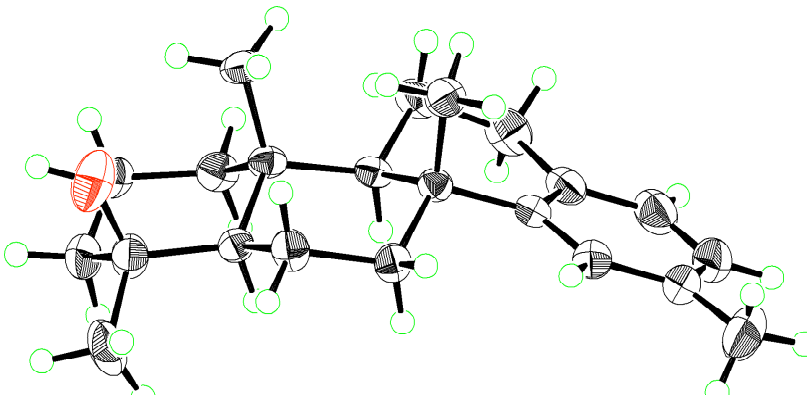

Figure 4. Molecular structure of desilylated $\mathbf{8 a} \beta$. Carbon, hydrogen and oxygen atoms are shown in black, green and red colors, respectively.

The relative stereochemistries of 11a $\alpha$ and 11a $\beta$ were determined based on the X-ray diffraction analysis of desilylated 11a $\beta$ (Figure 5). Other relative stereochemistries of $\mathbf{1 1} \alpha$ and $\mathbf{1 1} \beta$ were determined as analogues of 11a $\alpha$ and 11a $\beta$ in comparison with their ${ }^{1} \mathrm{H}$ and ${ }^{13} \mathrm{C}$ NMR spectra 


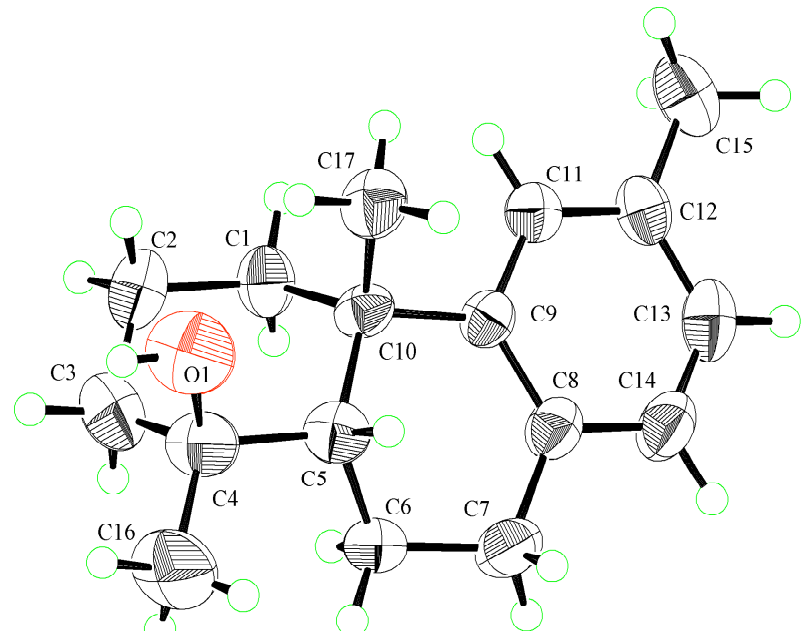

Figure 5. Molecular structure of desilylated 11a $\beta$. Carbon, hydrogen and oxygen atoms are shown in black, green and red colors, respectively.

The relative stereochemistries of $\mathbf{1 5} \alpha$ and $\mathbf{1 5} \beta$ were determined as analogues of $\mathbf{1 5 \alpha}\left(\mathrm{R}^{1}=\mathrm{H}\right)$ and $15 \beta\left(\mathrm{R}^{1}=\mathrm{H}\right)$ in comparison with their ${ }^{1} \mathrm{H}$ and ${ }^{13} \mathrm{C}$ NMR spectra. ${ }^{15}$

\section{X-Ray Diffraction Analysis of 5c $\beta$ and Desilylated 8a $\alpha, 8 a \beta$ and 11a $\beta$}

X-ray crystallographic analysis was performed with a Bruker SMART APEX diffractometer (graphite monochromator, MoK $\alpha$ radiation, $\lambda=0.71073 \AA$ ) and the structure was solved by direct methods and expanded using Fourier techniques (Sir97 and SHELXL ${ }^{16}$ ).

Recrystallization of $\mathbf{5 c} \boldsymbol{\beta}$ was carried out in the solution of $\mathrm{MeOH}$-hexane at room temperature (Table 5). Mp: $114-116{ }^{\circ} \mathrm{C}$. Crystallographic data have been deposited with Cambridge Crystallographic Data Centre: Deposition number CCDC 293029 for $\mathbf{5 c} \beta$. Copies of the data can be obtained free of charge via www.ccdc.cam.ac.uk/conts/retrieving.html (or from the Cambridge Crystallographic Data Centre, 12, Union Road, Cambridge, CB2 1EZ, UK; fax: +44 1223 336033; or deposit@ccdc.cam.ac.uk).

Table 5. Crystallographic Data and Structure Refinement for $5 c \beta$

\begin{tabular}{ll}
\hline Compound & $\mathbf{5 c \beta}$ \\
empirical formula & $\mathrm{C}_{26} \mathrm{H}_{44} \mathrm{OSi}$ \\
formula weight & 400.70 \\
$T$ & $223(2) \mathrm{K}$ \\
$\lambda$ & $0.71073 \AA$ \\
crystal system & triclinic \\
space group & $P 1$ \\
$A$ & $7.654(3) \AA$ \\
$B$ & $8.087(3) \AA$ \\
$C$ & $21.574(8) \AA$ \\
$\alpha$ & $89.127(7)^{\circ}$ \\
$\beta$ & $85.581(7)^{\circ}$ \\
$\gamma$ & $66.067(6)^{\circ}$ \\
$V$ & $1216.7(8) \AA^{3}$ \\
$Z$ & 2 \\
$D_{\text {calcd }}$ & $1.094 \mathrm{~g} / \mathrm{cm}^{3}$ \\
absorption coefficient & $0.110 \mathrm{~mm}^{-1}$ \\
$F(000)$ & 444 \\
crystal size & $0.20 \times 0.15 \times 0.15 \mathrm{~mm}^{3}$ \\
theta range for data collection & 1.89 to $29.22^{\circ}$ \\
reflections collected & 8913 \\
independent reflections & 6145 \\
$R_{\text {int }}$ & 0.0305 \\
refinement based on & $F^{2}$ \\
no. of data & 6145 \\
no. of parameters & 429
\end{tabular}




\begin{tabular}{ll} 
no. of restraints & 0 \\
$\mathrm{GOF}$ & 0.997 \\
$\mathrm{R}(F)$ for $I>2 \sigma(I)$ & 0.0541 \\
$\mathrm{wR} 2\left(F^{2}\right)$ for all data & 0.1513 \\
$\Delta \rho_{\min }$ & $-0.358 \mathrm{e}^{-3}$ \\
$\Delta \rho_{\max }$ & $0.536 \mathrm{e}^{-3}$ \\
\hline
\end{tabular}

Recrystallization of desilylated $\mathbf{8 a} \boldsymbol{\alpha}$ was carried out in wet acetone at room temperature (Table 6). Mp: $116-118{ }^{\circ} \mathrm{C}$. Crystallographic data have been deposited with Cambridge Crystallographic Data Centre: Deposition number CCDC 296031 for desilylated 8a $\alpha$. Copies of the data can be obtained free of charge via www.ccdc.cam.ac.uk/conts/retrieving.html (or from the Cambridge Crystallographic Data Centre, 12, Union Road, Cambridge, CB2 1EZ, UK; fax: +44 1223 336033; or deposit@ccdc.cam.ac.uk).

Table 6. Crystallographic Data and Structure Refinement for Desilylated 8a $\alpha$

\begin{tabular}{ll}
\hline Compound & Desilylated 8a $\alpha$ \\
empirical formula & $\mathrm{C}_{44} \mathrm{H}_{64} \mathrm{O}_{2}$ \\
formula weight & 624.95 \\
$T$ & $223(2) \mathrm{K}$ \\
$\lambda$ & $0.71073 \AA$ \\
crystal system & Triclinic \\
space group & $P 1$ \\
$A$ & $7.147(7) \AA$ \\
$b$ & $12.736(12) \AA$ \\
$C$ & $19.488(18) \AA$ \\
$\alpha$ & $90.337(19)^{\circ}$ \\
$\beta$ & $90.130(19)^{\circ}$ \\
$\gamma$ & $98.734(19)^{\circ}$ \\
$V$ & $1753(3) \AA^{3}$ \\
$Z$ & 2 \\
$D_{\text {calcd }}$ & $1.184 \mathrm{~g} / \mathrm{cm}^{3}$ \\
absorption coefficient & $0.070 \mathrm{~mm}^{-1}$ \\
$F(000)$ & 688 \\
crystal size & $0.20 \times 0.15 \times 0.15 \mathrm{~mm}^{3}$ \\
theta range for data collection & 1.04 to $29.35^{\circ}$ \\
reflections collected & 13039 \\
independent reflections & 8985 \\
$R_{\text {int }}$ & 0.0256 \\
refinement based on & $F^{2}$ \\
no. of data & 8985 \\
no. of parameters & 431 \\
no. of restraints & 0 \\
GOF & 0.964 \\
$\mathrm{R}(F)$ for $I>2 \sigma(I)$ & 0.0531 \\
wR2 $\left(F{ }^{2}\right)$ for all data & 0.1577 \\
$\Delta \rho_{\text {min }}$ & $-0.224 \mathrm{e}^{-3}$ \\
$\Delta \rho_{\text {max }}$ & $0.308 \AA^{-3}$ \\
\hline
\end{tabular}

Desilylated 8a $\beta$ was recrystallized in wet acetone at room temperature (Table 7). Mp: $103-$ $105{ }^{\circ} \mathrm{C}$. Crystallographic data have been deposited with Cambridge Crystallographic Data Centre: Deposition number $\underline{\text { CDC } 296030}$ for desilylated 8a $\beta$. Copies of the data can be obtained free of charge via www.ccdc.cam.ac.uk/conts/retrieving.html (or from the Cambridge Crystallographic Data Centre, 12, Union Road, Cambridge, CB2 1EZ, UK; fax: +44 1223 336033; or deposit@ccdc.cam.ac.uk).

Table 7. Crystallographic Data and Structure Refinement for Desilylated 8a $\beta$

\begin{tabular}{ll}
\hline Compound & Desilylated 8a $\beta$ \\
empirical formula & $\mathrm{C}_{88} \mathrm{H}_{140} \mathrm{O}_{10}$ \\
formula weight & 1358.00 \\
$T$ & $223(2) \mathrm{K}$
\end{tabular}




\begin{tabular}{ll}
$\lambda$ & $0.71073 \AA$ \\
crystal system & Monoclinic \\
space group & $P 21 / \mathrm{c}$ \\
$A$ & $21.254(6) \AA$ \\
$B$ & $7.386(2) \AA$ \\
$C$ & $25.122(7) \AA$ \\
$\alpha$ & $90^{\circ}$ \\
$\beta$ & $109.476(6)^{\circ}$ \\
$\gamma$ & $90^{\circ}$ \\
$V$ & $3718.2(19) \AA^{3}$ \\
$Z$ & 2 \\
$D_{\text {calcd }}$ & $1.213 \mathrm{~g} / \mathrm{cm}^{3}$ \\
absorption coefficient & $0.077 \mathrm{~mm}^{-1}$ \\
$F(000)$ & 1496 \\
crystal size & $0.20 \times 0.15 \times 0.12 \mathrm{~mm}^{3}$ \\
theta range for data collection & 1.68 to $29.23^{\circ}$ \\
reflections collected & 26715 \\
independent reflections & 9794 \\
$R_{\text {int }}$ & 0.0503 \\
refinement based on & $F^{2}$ \\
no. of data & 9794 \\
no. of parameters & 458 \\
no. of restraints & 0 \\
GOF & 1.020 \\
$\mathrm{R}(F)$ for $I>2 \sigma(I)$ & 0.0837 \\
wR2 $\left(F^{2}\right)$ for all data & 0.2853 \\
$\Delta \rho_{\min }$ & $-0.707 \mathrm{e} \AA^{-3}$ \\
$\Delta \rho_{\text {max }}$ & $0.747 \mathrm{e}^{-3}$ \\
\hline
\end{tabular}

Recrystallization of desilylated 11a $\beta$ was carried out in chloroform at room temperature (Table 8). Mp: 90-93 ${ }^{\circ} \mathrm{C}$. Crystallographic data have been deposited with Cambridge Crystallographic Data Centre: Deposition number $\underline{\text { CDC } 296032}$ for desilylated 11a $\beta$. Copies of the data can be obtained free of charge via www.ccdc.cam.ac.uk/conts/retrieving.html (or from the Cambridge Crystallographic Data Centre, 12, Union Road, Cambridge, CB2 1EZ, UK; fax: +44 1223 336033; or deposit@ccdc.cam.ac.uk).

Table 8. Crystallographic Data and Structure Refinement for Desilylated 11a $\beta$

\begin{tabular}{ll}
\hline Compound & desilylated 11a $\beta$ \\
empirical formula & $\mathrm{C}_{17} \mathrm{H}_{24} \mathrm{O}$ \\
formula weight & 244.36 \\
$T$ & $223(2) \mathrm{K}$ \\
$\lambda$ & $0.71073 \AA$ \\
crystal system & monoclinic \\
space group & $P 21 / \mathrm{c}$ \\
$A$ & $14.862(4) \AA$ \\
$B$ & $7.073(2) \AA$ \\
$C$ & $13.688(4) \AA$ \\
$\alpha$ & $90^{\circ}$ \\
$\beta$ & $104.242(7)^{\circ}$ \\
$\gamma$ & $90^{\circ}$ \\
$V$ & $1394.6(7) \AA^{3}$ \\
$Z$ & 4 \\
$D_{\text {calcd }}$ & $1.164 \mathrm{~g} / \mathrm{cm}^{3}$ \\
absorption coefficient & $0.070 \mathrm{~mm}^{-1}$ \\
$F(000)$ & 536 \\
crystal size & $0.40 \times 0.20 \times 0.20 \mathrm{~mm}^{3}$ \\
theta range for data collection & 2.83 to $29.16^{\circ}$ \\
reflections collected & 9990 \\
independent reflections & 3699 \\
$R_{\text {int }}$ & 0.0387 \\
refinement based on & $F^{2}$ \\
no. of data & 3699 \\
no. of parameters & 170 \\
no. of restraints & 0 \\
GOF & 1.076 \\
$\mathrm{R}(F)$ for $I>2 \sigma(I)$ & 0.0849 \\
wR2 $\left(F^{2}\right)$ for all data & 0.2970 \\
\hline &
\end{tabular}




\begin{tabular}{ll}
$\Delta \rho_{\min }$ & $-0.245 \mathrm{e}^{\AA^{-3}}$ \\
$\Delta \rho_{\max }$ & $0.639 \AA^{-3}$ \\
\hline
\end{tabular}

\section{References and Notes}

$\dagger$ Nagoya University

* The University of Chicago

1. (a) Dodd, D. S; Oehlschlager, A. C. J. Org. Chem. 1992, 57, 7226-7234. (b) Sakaguchi, S.; Nishiyama, Y; Ishii, Y. J. Org. Chem. 1996, 61, 5307-5311.

2. Fillion, E.; Beingessner, R. L. J. Org. Chem. 2003, 68, 9485-9488.

3. (a) Corey, E. J.; Kania, R. S. J. Am. Chem. Soc. 1996, 118, 1229-1230. (b) Barrero, A. F.: Herrador, M. M.; del Moral, J. F. Q.; Arteaga, P.; Arteaga, J. F.; Piedra, M.; Sanchez, E. M. Org. Lett. 2005, 7, 2301-2304.

4. Trost, B. M.; Machacek, M. R.; Tsui, H. C. J. Am. Chem. Soc. 2005, 127, 7014-7024.

5. Danheiser, R. L.; Gee, S. K.; Perez, J. J. J. Am. Chem. Soc. 1986, 108, 806-810.

6. (a) Eric, F.; Rachel, L. B. J. Org. Chem. 2003, 68, 9485-9488. (b) Yanagisawa, A.; Noritake, Y.; Yamamoto, H. Chem. Let. 1988, 1899-1902.

7. Cane, D. E.; Iyengar, R.; Shiao, M.-S. J. Am. Chem. Soc. 1981, 103, 914-931.

8. Kim, G. T.; Wenz, M.; Park, J. I.; Hasserodt, J. K.; Janda, D. Biorg. Med. Chem. 2002, 10, 12491262.

9. Araki, S.; Sato, T.; Butsugan, Y. J. Chem. Soc. Chem. Commun. 1982, 285.

10. (a) Abouabdellah, A.; Bonnet-Delpon, D. Tetrahedron 1994, 50, 11921-11932. (b) Dukink, J.; Speckamp, W. N. Tetrahedron 1978, 34, 173-178.

11. (a) Dakir, M.; El Hanbali, F.; Mellouki, F.; Akssira, M.; Benharref, A.; Quilez del Moral, J. F.; Barrero, A. F. Nat. Pro. Res. 2005, 19, 719-722. (b) Kuo, Y.-H.; Yeh, M.-H. Phtochem. 1998, 49, 2453-2455. (c) Shmidt, E. N.; Chernenko, G. F.; Ivanova, E. E.; Demenkova, L. I. Khim. Prirod. Soed. 1996, 4, 608-609. (d) Lee, C.-K.; Fang, J. M. Cheng, Y. S. Phytochem. 1995, 39, 391-394. (e) Tkachev, A. V.; Denisov, A. Y. Tetrahedron 1994, 50, 2591-2598. (f) Barton, D. H. R.; Bridon. D.; Zar, S. Z. Tetrahedron 1989, 45, 2615-2626. (g) Zhogal'skii, A. N.; Azarko, V. A.; Mitskevich, N. I. Sery. Khim. Navuk 1983, 5, 14-17. (h) Rezvukhin, A. I.; Solomonnikova, I. V.; Schmidt, E. N. Ser. Khimich. 1979, 12, 2678-2685. (i) Okuda, S.; Sanai, S.; Tahara, A. Nippon Nogei Kagaku Kaishi 1977, 51, 227-232. (j) Okuda, S.; Sanai, S.; Kimura, Y.; Tamura, S.; Tahara, A. Agric. Bio. Chem. 1976, 40, 1327-1334. (k) Caputo, R.; Previtera, L.; Monaco, P.; Mangoni, L. Tetrahedron 1974, 30, 963-967. (1) Avdyukova, N. V.; Shmidt, E. N.; Pentagova, V. A. Ser. Khim. Nauk 1973, 140-142. (m) Carman, R. M. Aust. J. Chem. 1973, 26, 879-881. (n) Avdyukova, N. V.; Shmidt, E. N.; Pentagova, V. A. Ser. Khim. Nauk 1973, 117-120. (o) Avdyukova, N. V.; Raldugin, V. A.; Shmidt, E. N.; Pentagova, V. A. Khim. Prir. Soed. 1972, 5, 653-654. (p) Morozkov. V. K.; Shmidt, E. N.; Pentagova, V. A. Ser. Khim. Nauk 1972, 128-34. (q) Rowe, J. W.; Nagasampagi, B. A.; Burgstahler, A. W.; Fitzsimmons, J. W. Phytochem. 1971, 10, 16471651. (r) Bennet, C. R.; Cambie, R. C.; Franich, R. A.; Fullerton, T. J. Aust. J. Chem. 1969, 22 , 1711-1720. (s) Seelye, R. N.; Watkins, W. B. Tetrahedron Lett. 1968, 10, 1271-1272. (t) Huffman, J. W.; Stockel, R. F. J. Org. Chem. 1963, 28, 506-509.

12. (a) Tagat, J. R.; Nazareno, D. V.; Puar, M. S.; McCombie S. W.; Ganguly, A. K. Bioorg. Med. Chem. Lett. 1994, 4, 1101-1104. (b) Akita, H.; Otsuka, Y. Jpn. Kokai Tokkyo Koho 1976, JP 51098258. (c) Akita, H.; Otsuka, Y. Jpn. Kokai Tokkyo Koho 1976, JP 51098267. (d) Hoehn, W. M. 1954, US 2676183. (e) Stork, G.; Burgstahler, A. J. Am. Chem. Soc. 1951, 73, 3544-3546.

13. (a) Ley, S. V.; Griffith, W. P.; Marsden, S. P. Synthesis 1994, 639-666. (b) Gool, M. V.; Vandewalle, M. Eur. J. Org. Chem. 2000, 3427-3431.

14. (a) Bie, P.-Y.; Zhang, C.-L.; Peng, X.-J.; Chen, B.; Yang, Y.; Pan, X.-F. Gaodeng Xuexiao Huaxue Xuebao 2003, 24, 1219-1221. (b) Kutney, J. P.; Han, K.; Kuri-B., F.; Milanova, R. K.; Roberts, M. 
Heterocycles 1997, 44, 95-104. (c) Ref 3a. (d) Thompson, H. W.; Long, D. J. J. Org. Chem. 1988, 53, 4201-4209. (e) Huffman, J. W.; Harris, P. G. J. Org. Chem. 1977, 42, 2357-2362. (f) Tahara, A.; Harigaya, Y.; Onda, M. Chem. Pharm. Bull. 1976, 24, 427-430. (g) Bennet, C. R.; Cambie, R. C.; Franich, R. A.; Fullerton, T. J. Aust. J. Chem. 1969, 22, 1711-1720. (h) Sharma, M.; Ghatak, U. R.; Dutta, P. C. Tetrahedron 1963, 19, 985-994. (i) Huffman, J. W.; Stockel, R. F. J. Org. Chem. 1963, 28, 506-509. (j) Jacobsen, R. P. 1957, US 2781401. (k) Arigoni, D.; Kalvoda, J.; Heusser, H.; Jeger, O; Ruzicka, L. Helv. Chim. Acta 1955, 38, 1857-1862. (l) Hoehn, W. M. 1954, US 2681367. (m) Hoehn, W. M. 1954, US 2676184. (n) Zeiss, H. H.; Martin, W. B., Jr. J. Am. Chem. Soc. 1953, 75, 5935-5940.

15. Gonzalez-Sierra, M.; Leader, H. N.; McChesney, J. D. J. Org. Chem. 1985, 50, 4450-4456.

16. Sheldrick, G. M. SHELXL-97, Program for Crystal Structure Refinement; University of Göttingen: Göttingen, Germany, 1997. 